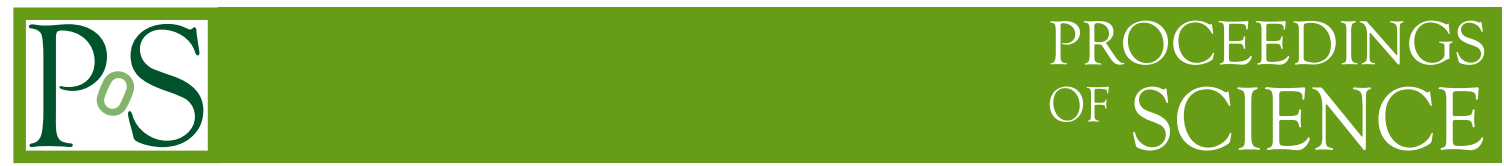

\title{
Lectures on Integrable Systems
}

\section{Gleb Arutyunov ${ }^{a * *}$}

${ }^{a}$ II. Institut für Theoretische Physik, Universität Hamburg, Luruper Chaussee 149, 22761 Hamburg, Germany

Zentrum für Mathematische Physik, Universität Hamburg,

Bundesstrasse 55, 20146 Hamburg, Germany

E-mail: gleb.arutyunov@desy.de

In these lectures we cover the basic concepts of integrability including elements of the geometric approach based on the hamiltonian reduction, the classical and quantum factorised scattering theory and the coordinate Bethe Ansatz. The main features of the formalism are demonstrated on the examples of the Lieb-Liniger delta-interaction model and multi-body systems of the CalogeroMoser-Sutherland type.

RDP online workshop "Recent Advances in Mathematical Physics" - Regio2020,

5-6 December 2020

online

${ }^{*}$ Speaker 


\section{Contents}

Preface 2

1 Liouville integrable systems 4

1.1 Dynamical systems in classical mechanics 4

$\begin{array}{lll}1.2 & \text { Liouville theorem } & 11\end{array}$

1.3 Some examples of integrable systems 17

1.4 Lax representation and classical $r$-matrix 22

2 Phase spaces with symmetries and reduction 28

2.1 Lie-Poisson structure and coadjoint orbits 28

2.2 Hamiltonian reduction 31

2.3 Hyperbolic CMS model from reduction 37

3 Quantum integrability I: Discrete spectrum 41

3.1 Quantum trigonometric CMS model 41

3.2 Spectrum via Jack polynomials 44

4 Quantum integrability II: Scattering and Bethe Ansatz 47

$\begin{array}{lll}4.1 & \text { Scattering in classical integrable models } & 47\end{array}$

4.2 Factorisation of scattering matrix 51

$\begin{array}{lll}4.3 & \text { Bethe-Yang equations } & 57\end{array}$

5 Two-dimensional integrable PDEs

$\begin{array}{lll}5.1 & \text { Soliton solutions } & 62\end{array}$

$\begin{array}{lll}5.2 & \text { Zero curvature representation } & 67\end{array}$

5.3 Local integrals of motion $\quad 69$

\section{Preface}

This is a mini course on integrable systems given at the Yerevan Physics Institute in February 2020. The course is of an introductory nature and we tried to keep the presentation at the level assessable by graduate students and postdocs.

We start the discussion with recalling the basic notions of classical mechanics including the elements of Poisson and symplectic geometry. This creates an environment conducive to formulate the Liouville theorem and outline its proof. For a mechanical system that satisfies the requirements of the Liouville theorem, equations of motion can in principle be solved by means of a special 
procedure - the "quadrature", although a practical implementation of this procedure often meets severe difficulties. We then give a few examples of finite-dimensional integrable models which will serve throughout the lectures to demonstrate various methods and techniques aimed at finding their solutions. These models include the $\mathrm{SO}(n)$ generalisation of Eulers' top, the CalogeroMoser-Sutherland (CMS) multi-body systems and the Lieb-Liniger model for the Bose gas. The contemporary approach to integrable models relies on the Lax representation of the equations of motion that provides an explicit construction of integrals of motion. The involutive property of these integrals, hence the Liouville integrability, is guaranteed by a special form of the Poisson bracket between the components of the Lax matrix. This brings forward the notion of the classical $r$-matrix - a ubiquitous structure in the modern theory of integrable models. In some cases, however, the Lax representation fails to generate the necessary number of integrals to render a dynamical system Liouville integrable, as it happens, for instance, for Euler's top. In this situation the Lax representation and the procedure of constructing integrals can be generalised to include the dependence on an auxiliary (spectral) parameter and in this way to overcome the above-mentioned difficulty.

The next topic concerns with a geometric origin of integrable models. In many important cases these models can be constructed by means of the hamiltonian reduction procedure. At the same time, this procedure reduces the problem of solving the corresponding differential equations of motion to a certain factorisation problem in Lie algebras and Lie groups that can be further solved by means of algebraic operations. The starting point of this approach is a symplectic manifold that carries a symplectic action of a Lie group $G$. One can then construct in a canonical way a new (reduced) manifold, smaller in dimension, which, according to the Marsden-Weinstein theorem, is also symplectic with the symplectic structure inherited from the parent manifold. A dynamical system with a $G$-invariant hamiltonian on the parent manifold descends on the reduced manifold where, under certain conditions, it defines an integrable model. To describe the reduction procedure in full detail, we start from recalling the theory of coadjoint orbits, the Lie-Poisson structure, symplectic action of a Lie group and the notion of moment map. This brings us to the Marsden-Weinstein theorem and the discussion of the reduced symplectic structure. As an illustration of the hamiltonian reduction procedure, we apply it to construct the hyperbolic CMS model by reducing the cotangent bundle $\mathrm{T}^{*} G$ over the adjoint action of $G$ and show how to solve the equations of motion of this model by means of a special factorisation in $G$.

Further we proceed with quantum integrable systems. Given a classical integrable system with independent involutive integrals $H_{k}$, its quantum-mechanical version should admit the quantum counterparts $\widehat{H}_{k}$ that all pairwise commute and, for this reason, share a common spectrum. The multi-body wave function can thus be searched as a common eigenstate of these operators. The study of models with discrete or continuous spectrum of the Schrödinger operator proceeds differently. For the discrete case, rather than to develop any general scheme, we consider a concrete model, namely, the quantum version of the trigonometric CMS model. Analysing the action of the corresponding hamiltonian on symmetric functions of exponentiated coordinates, we explain how to diagonalise it on the basis of Jack polynomials. Further on, we discuss the quasi-particle interpretation of the spectrum and its encoding by the corresponding Baxter polynomial.

The case of models with continuous spectrum is naturally related to the scattering theory. We first embark on scattering in classical mechanics by restricting ourselves for simplicity reasons 
to repulsive, impenetrable potentials, rapidly decreasing at infinity. A new feature brought by integrability in the scattering picture is the conservation of a set of asymptotic momenta, which essentially means that scattering in integrable models is non-diffractive. In classical theory, the main characteristic of scattering is the phase shift which shows how much a particle is advanced with respect to a would be freely moving particle with the same asymptotic momentum. We then discuss a general formula expressing the phase shift via the two-body potential and apply it to the rational and trigonometric CMS models.

The conservation of the set of asymptotic momenta pertains to quantum integrable models admitting scattering. Moreover, this conservation restricts the form of the asymptotics of the wave function at large separation of neighbouring particles to be a linear combination of plane waves constructed from the one and the same set of asymptotic momenta. This special form of the asymptotic wave function is known as the Bethe wave function. The coefficients in the expansion of the Bethe wave function over plane waves can be expressed via the two-body Smatrix. Integrability then implies a factorisation of the multi-body scattering into a product of two-body events and requires the two-body S-matrix to satisfy a compatibility condition known as the quantum Yang-Baxter equation.

Although for most of integrable models the Bethe form of the wave function is only asymptotic, this form can still be used to gain the knowledge of the spectrum when a model is compactified on a large circle. This amounts to subjecting the Bethe wave function to periodicity conditions that result into quantisation conditions for particle momenta known as Bethe equations. As an illustration, we present the Bethe equations and the wave function for the cases of the Lieb-Liniger model of the Bose gas and the rational CMS model. Actually, in the first case the Bethe wave function gives an exact solution of the problem due to the extreme short-ranginess of the potential, while in the second case the Bethe equations can be solved exactly and the corresponding spectrum appears to coincide with the exact spectrum of the trigonometric CMS model found from solving the latter model in terms of Jack polynomials.

In this course we decided to mainly talk about finite-dimensional integrable models. This decision is based on our educative experience that once the Bethe Ansatz approach for the asymptotic spectrum of a quantum-mechanical integrable model is understood, it does not take much effort to adapt it for the field-theoretic case. Notwithstanding, in the last part of the course we offer a glimpse on the theory of two-dimensional integrable PDEs. Admittedly, this is a very vast subject on its own, so to keep the size of our presentation within reasonable, we restricted ourselves to stressing only some basic features, such as an existence of soliton solutions and the zero curvature representation.

\section{Liouville integrable systems}

Traditionally, we start with a brief overview the basic notions of classical mechanics and a digression on the Liouville theorem.

\subsection{Dynamical systems in classical mechanics}

There are two ways to describe dynamical systems in classical mechanics [1]. The first description is known as the lagrangian formalism based on the principle of "stationary action". Consider a point 
particle with mass $m$ which moves in an $N$-dimensional space with coordinates $q=\left(q^{1}, \ldots, q^{N}\right)$ and a potential $V(q)$. Newton's equations which govern the particle's trajectory are

$$
m \ddot{q}^{i}=-\frac{\partial V}{\partial q^{i}} .
$$

These equations can be obtained by extremising the following action functional

$$
S[q]=\int_{t_{1}}^{t_{2}} \mathrm{~d} t L(q, \dot{q}, t)=\int_{t_{1}}^{t_{2}} \mathrm{~d} t\left(\frac{m \dot{q}^{2}}{2}-V(q)\right) .
$$

According to the principle of stationary action, the actual trajectories of a dynamical system are the ones that extremise $S$.

In general, we consider the lagrangian $L$ as an arbitrary function of $q, \dot{q}$ and time $t$. The equations of motion are obtained by extremising the corresponding action

$$
\frac{\delta S}{\delta q^{i}}=\frac{\partial L}{\partial q^{i}}-\frac{d}{d t}\left(\frac{\partial L}{\partial \dot{q}^{i}}\right)=0
$$

and they are called the Euler-Lagrange equations. An assumption that $L$ does not involve higher order time derivatives implies that the corresponding dynamical system is fully determined by specifying initial coordinates and velocities. Indeed, for a system with $N$ degrees of freedom there are $N$ Euler-Lagrange equations of second order; the general solution will depend on $2 N$ integration constants, which are determined by specifying e.g. the initial coordinates and velocities. Note that adding to the lagrangian a time derivative of a function which depends on coordinates and time only: $L \rightarrow L+\frac{d}{d t} \Lambda(q, t)$ will not influence the Euler-Lagrange equations.

If $L$ does not explicitly depend on $t$, then $\frac{d L}{d t}=\frac{\partial L}{\partial \dot{q}^{i}} \ddot{q}^{i}+\frac{\partial L}{\partial q^{i}} \dot{q}^{i}$. Substituting here $\frac{\partial L}{\partial q^{i}}$ from the Euler-Lagrange equations, we get

$$
\frac{d L}{d t}=\frac{\partial L}{\partial \dot{q}^{i}} \ddot{q}^{i}+\frac{d}{d t}\left(\frac{\partial L}{\partial \dot{q}^{i}}\right) \dot{q}^{i}=\frac{d}{d t}\left(\frac{\partial L}{\partial \dot{q}^{i}} \dot{q}^{i}\right) .
$$

Therefore,

$$
\frac{d}{d t}\left(\frac{\partial L}{\partial \dot{q}^{i}} \dot{q}^{i}-L\right)=0
$$

as a consequence of the equations of motion. This means that the quantity

$$
H \equiv \frac{\partial L}{\partial \dot{q}^{i}} \dot{q}^{i}-L
$$

is conserved under the time evolution. For our particular example,

$$
H=m \dot{q}^{2}-L=\frac{m \dot{q}^{2}}{2}+V(q)=E,
$$

where $\dot{q}^{2} \equiv \dot{q}^{i} \dot{q}^{i}$. Thus, $H$ is nothing else but the energy $E$ of the system; the energy is conserved due to the equations of motion. In general, dynamical quantities which remain unchanged under the time evolution are called conservation laws or integrals of motion. Conservation of energy is one of the main examples of conservation laws. 
Introduce the quantity called the canonical momentum

$$
p_{i}=\frac{\partial L}{\partial \dot{q}^{i}}, \quad p=\left(p_{1}, \ldots, p_{N}\right) .
$$

Obviously, for a particle $p_{i}=m \dot{q}^{i}$. If $V=0$, then $\dot{p}_{i}=0$ by the Euler-Lagrange equations. Thus, in the case of vanishing potential, the particle momentum is an integral of motion. This is another example of a conservation law.

The second description of dynamical systems exploits the notion of the hamiltonian. The energy of a system expressed via canonical coordinates and momenta is called the hamiltonian:

$$
H(p, q)=\frac{p^{2}}{2 m}+V(q)
$$

where $p^{2} \equiv p_{i} p_{i}$. Given the hamiltonian, Newton's equations can be rewritten as

$$
\dot{q}^{j}=\frac{\partial H}{\partial p_{j}}, \quad \dot{p}_{j}=-\frac{\partial H}{\partial q^{j}} .
$$

These are equations of motion in the hamiltonian form or Hamilton's equations. These equations can also be obtained by means of the variational principle. The corresponding action has the form, $c f .(1.2)$ and (1.3),

$$
S[p, q]=\int_{t_{1}}^{t_{2}}\left(p_{i} \dot{q}^{i}-H(p, q)\right) \mathrm{d} t .
$$

Varying this action with respect to $p$ and $q$, considered as independent variables, one obtains Hamilton's equations.

Hamilton's equations can be represented in the form of a single equation. Introduce two $2 N$-dimensional vectors

$$
x=\left(\begin{array}{c}
q \\
p
\end{array}\right), \quad \nabla H=\left(\begin{array}{c}
\frac{\partial H}{\partial q^{j}} \\
\frac{\partial H}{\partial p_{j}}
\end{array}\right)
$$

and $2 N \times 2 N$ matrix $J$ :

$$
J=\left(\begin{array}{rr}
0 & -\mathbb{1} \\
\mathbb{1} & 0
\end{array}\right),
$$

where $\mathbb{1}$ is the $N \times N$ unit matrix. Then (1.4) are concisely written as

$$
\dot{x}=-J \cdot \nabla H, \quad \text { or } \quad J \cdot \dot{x}=\nabla H .
$$

The point $x=\left(x^{1}, \ldots, x^{2 N}\right)$ defines a state of a dynamical system in classical mechanics. The set of all states forms the phase space $\mathscr{P}=\{x\}$ of the system which in the present case is the $2 \mathrm{~N}$-dimensional space with the euclidean metric. Solving Hamilton's equations with given initial conditions $\left(p_{0}, q_{0}\right)$ representing a point in the phase space, we obtain a phase space curve

$$
p \equiv p\left(t ; p_{0}, q_{0}\right), \quad q \equiv q\left(t ; p_{0}, q_{0}\right)
$$


passing through this point. As follows from the uniqueness theorem for ordinary differential equations, there is a unique phase curve through every phase space point.

Let $\mathscr{F}(\mathscr{P})$ be the space of smooth real-valued functions on $\mathscr{P}$. It carries the structure of an algebra with respect to the pointwise multiplication and its elements are called observables. Using the matrix $J$, one can define on $\mathscr{F}(\mathscr{P})$ the following Poisson bracket

$$
\{f, g\}(x)=J^{i j} \partial_{i} f \partial_{j} g=\sum_{i=1}^{N}\left(\frac{\partial f}{\partial p_{i}} \frac{\partial g}{\partial q^{i}}-\frac{\partial f}{\partial q^{i}} \frac{\partial g}{\partial p_{i}}\right)
$$

for any $f, g \in \mathscr{F}(\mathscr{P})$. The Poisson bracket is a map $\mathscr{F}(\mathscr{P}) \times \mathscr{F}(\mathscr{P}) \rightarrow \mathscr{F}(\mathscr{P})$ which has the following properties

1) Linearity $\{f+\alpha h, g\}=\{f, g\}+\alpha\{h, g\}$;

2) Skew-symmetry $\{f, g\}=-\{g, f\}$;

3) Jacobi identity $\{f,\{g, h\}\}+\{g,\{h, f\}\}+\{h,\{f, g\}\}=0$;

4) Leibniz rule $\{f, g h\}=\{f, g\} h+g\{f, h\}$

for arbitrary functions $f, g, h \in \mathscr{F}(\mathscr{P})$ and $\alpha \in \mathbb{R}$. The first three properties imply that the Poisson bracket introduces on $\mathscr{F}(\mathscr{P})$ the structure of an infinite-dimensional Lie algebra, while the Leibniz rule expresses the compatibility of the bracket with multiplication in $\mathscr{F}(\mathscr{P})$. Due to this rule, the bracket is fully determined by its values on the coordinate functions $x^{i}$ for which $\left\{x^{i}, x^{j}\right\}=J^{i j}$ or, explicitly,

$$
\left\{q^{i}, q^{j}\right\}=0, \quad\left\{p_{i}, p_{j}\right\}=0, \quad\left\{p_{i}, q^{j}\right\}=\delta_{i}^{j} .
$$

Using the Poisson bracket, Hamilton's equations for the coordinate functions can be rephrased in the following concise form

$$
\dot{x}^{j}=\left\{H, x^{j}\right\} .
$$

As a consequence, evolution of any function $f$ on the phase space is governed by the equation

$$
\dot{f}=\{H, f\} .
$$

Due to the skew-symmetry property of the Poisson bracket, this form of Hamilton's equations makes the conservation law for $H$ obvious.

Poisson and symplectic manifolds. The properties 1) - 4) provide a general definition of the Poisson bracket for an arbitrary smooth manifold $\mathscr{P}$. Any Poisson bracket is described by a skewsymmetric tensor $J$ on $\mathscr{P}$ satisfying the Jacoby identity. In local coordinates this identity takes the form

$$
\sum_{(i, l, m)} J^{i k} \partial_{k} J^{l m}=0
$$

where the sum is over the cyclic permutation of indices. A manifold endowed with a Poisson bracket is called Poisson. 
For later we will need the notion of a Poisson map. For Poisson manifolds $\mathscr{M}$ and $\mathscr{N}$, a smooth map $\varphi: \mathscr{M} \rightarrow \mathscr{N}$ is called Poisson, if for any $f, h \in \mathscr{F}(\mathscr{N})$

$$
\{f, h\}_{\mathscr{N}}(\varphi(x))=\left\{\varphi^{*} f, \varphi^{*} h\right\}_{\mathscr{M}}(x),
$$

where $\varphi^{*} f(x)=f(\varphi(x))$ and $\varphi^{*} h(x)=h(\varphi(x)), x \in \mathscr{M}$, are pullbacks of $f$ and $h$. Here $\{,\}_{\mathscr{M}}$ and $\{,\}_{\mathscr{N}}$ stand for the Poisson brackets on the respective manifolds.

In general, the rank $r$ of the matrix $J$ is less than or equal to the dimension $\operatorname{dim} \mathscr{P}$ of a manifold and it might change from point to point. In the case when $r=\operatorname{dim} \mathscr{P}$ at every point, the matrix $J$ is invertible and the corresponding Poisson bracket is called non-degenerate. This is only possible if $\operatorname{dim} \mathscr{P}$ is even. Indeed, since $J^{t}=-J$, one has

$$
\operatorname{det} J=\operatorname{det}(-J)=(-1)^{\operatorname{dim} \mathscr{P}} \operatorname{det} J,
$$

so that $(-1)^{\operatorname{dim} \mathscr{P}}=1$ since $\operatorname{det} J \neq 0$.

A manifold $\mathscr{P}$ supplied with a non-degenerate Poisson bracket is called symplectic. The inverse of $J$ with entries $\omega_{i j}$, where $J^{i k} \omega_{k j}=\delta_{j}^{i}$, defines a skew-symmetric bilinear differential 2-form $\omega$ on $\mathscr{P}$

$$
\omega=-\frac{1}{2} \omega_{i j}(x) d x^{i} \wedge d x^{j} .
$$

The Jacobi identity for $J$ implies that this form is closed, i.e. $d \omega=0$. To show this, we first note that the Jacobi identity implies the following identity for $J$

$$
J^{i k} \partial_{k} J^{l m}+J^{m k} \partial_{k} J^{i l}+J^{l k} \partial_{k} J^{m i}=0 .
$$

Then, we multiply both sides by $\omega_{i j} \omega_{m s}$ and use $J^{i k} \omega_{k j}=\delta_{j}^{i}$ to obtain

$$
-\partial_{j} J^{m l} \omega_{m s}-\omega_{i j} \partial_{k} J^{i l}+\omega_{i j} J^{l k} \partial_{k} J^{m i} \omega_{m s}=0 .
$$

In the last relation we put derivatives on $\omega$ by using $\partial_{s} J^{i k} \omega_{k j}+J^{i k} \partial_{s} \omega_{k j}=0$. This gives

$$
J^{l m} \partial_{j} \omega_{m s}-J^{l i} \partial_{s} \omega_{i j}-J^{l m} \partial_{m} \omega_{j s}=0 .
$$

It remains to multiply the last relation by $\omega_{k l}$ and get

$$
\partial_{i} \omega_{j k}+\partial_{k} \omega_{i j}+\partial_{j} \omega_{k i}=0
$$

which is equivalent to

$$
\partial_{[i} \omega_{j k]}=0 \quad \longrightarrow d \omega=0 .
$$

A non-degenerate closed 2-form is called symplectic.

An example of a symplectic manifold is the space $\mathbb{R}^{2 N}$ with the bracket (1.7). The corresponding symplectic form is

$$
\omega=d p_{i} \wedge d q^{i}=d\left(p_{i} d q^{i}\right) .
$$

The 1-form $\alpha=p_{i} d q^{i}$ is called the canonical 1-form.

Given a Poisson manifold, to any function $f \in \mathscr{F}(\mathscr{P})$ one can associate a vector field $\xi_{f}$ defined as

$$
\xi_{f}=\{f, \cdot\}
$$


This field is called the hamiltonian vector field generated by $f$, and $f$ is the generating or hamiltonian function of $\xi_{f}$. In local coordinates $x^{i}$ we have

$$
\xi_{f}=J^{i j} \partial_{i} f \partial_{j}
$$

If we let $\xi_{f}=\xi_{f}^{j} \partial_{j}$, then the relation above gives

$$
\xi_{f}^{j}=J^{i j} \partial_{i} f, \quad \partial_{j} f=\omega_{i j} \xi_{f}^{i} .
$$

The Jacobi identity for the Poisson bracket implies

$$
\xi_{\{f, g\}}=\left[\xi_{f}, \xi_{g}\right] .
$$

Hence, the map $f \rightarrow \xi_{f}$ is a homomorphism ${ }^{1} \mathscr{F}(\mathscr{P}) \rightarrow \mathfrak{X}(\mathscr{P})$, where $\mathfrak{X}(\mathscr{P})$ is the Lie algebra of vector fields on $\mathscr{P}$.

If $\mathscr{P}$ is symplectic, the definition (1.9) of the hamiltonian vector field can be formulated with the help of the interior product $i_{\xi}$

$$
i_{\xi_{f}} \omega+d f=0
$$

while the one-to-one correspondence between the Poisson bracket and the symplectic form $\omega$ can be expressed as

$$
\omega\left(\xi_{f}, \xi_{h}\right)=\{f, h\}=\xi_{f} h=-\xi_{h} f .
$$

A function $C$ is called a central or Casimir function if it Poisson-commutes with any element of $\mathscr{F}(\mathscr{P})$, that is

$$
\{C, f\}=0, \quad \forall f \in \mathscr{F}(\mathscr{P}) .
$$

Casimir functions form a ring. If $C$ is a Casimir function then it is annihilated by any hamiltonian vector field $\xi_{f}$, i.e. the latter lies everywhere tangent to the level set of the function $C$. On the other hand, the hamiltonian vector field $\xi_{C}$ vanishes as the one-form $d C$ belongs to the kernel of $J: J d C=0$. Thus, the existence of non-constant Casimir functions means that $r \neq \operatorname{dim} \mathscr{P}$, i.e. the Poisson bracket is degenerate.

Let $\left\{C_{i}\right\}, i=1, \ldots, m$, be a complete set of independent Casimir functions. Consider a level set $\mathscr{P}_{c}=\left\{x \in \mathscr{P}: C_{i}(x)=c_{i}\right\}$, where $c_{i}$ are constants. Any hamiltonian vector field is tangent to $\mathscr{P}_{c}$

$$
\xi_{f} C_{i}=\left\{f, C_{i}\right\}=0, \quad \forall f \in \mathscr{F}(\mathscr{P}) .
$$

The same is true for the commutator of any two hamiltonian vector fields. Thus, by the Frobenius theorem, the level set $\mathscr{P}_{c}$ is an integral submanifold in $\mathscr{P}$. On $\mathscr{P}_{c}$ one can naturally define a 2-form $\omega$

$$
\omega_{x}\left(\xi_{f}, \xi_{g}\right)=\{f, g\}(x), \quad x \in \mathscr{P}_{c},
$$

\footnotetext{
${ }^{1}$ The map is from functions into vector fields, not vice versa, because functions which differ by a constant have, in fact, the one and the same hamiltonian vector field.
} 
where $\omega_{x}$ is the value of $\omega$ at $x$. The differential of $\omega$ can be computed with the help of the formula ${ }^{2}$

$$
\begin{aligned}
3 d \omega\left(\xi_{f}, \xi_{g}, \xi_{h}\right) & =\xi_{f} \omega\left(\xi_{g}, \xi_{h}\right)+\xi_{g} \omega\left(\xi_{h}, \xi_{f}\right)+\xi_{h} \omega\left(\xi_{f}, \xi_{g}\right) \\
& -\omega\left(\left[\xi_{f}, \xi_{g}\right], \xi_{h}\right)-\omega\left(\left[\xi_{h}, \xi_{f}\right], \xi_{g}\right)-\omega\left(\left[\xi_{g}, \xi_{h}\right], \xi_{f}\right) .
\end{aligned}
$$

Using (1.11), definition (1.14) and the Jacobi identity, we get $d \omega=0$. Since the hamiltonian vector field of any Casimir function vanishes, the form $\omega$ is non-degenerate and, therefore, it is symplectic, i.e. $\mathscr{P}_{c}$ is a symplectic manifold. Thus, the hamiltonian vector fields foliate $\mathscr{P}$ into integral even-dimensional sub-manifolds called symplectic leaves, each of which inherits a symplectic form from the original Poisson bracket on $\mathscr{P}$.

Canonical transformations. Consider a smooth coordinate transformation $x \rightarrow x^{\prime}=x^{\prime}(x)$. In terms of these new coordinates Hamilton's equations (1.6) take the form

$$
\frac{d x^{i}}{d t}=\frac{\partial x^{\prime i}}{\partial x^{k}} \frac{d x^{k}}{d t}=\frac{\partial x^{\prime i}}{\partial x^{k}} J^{k m}(x) \nabla_{m}^{x} H=\frac{\partial x^{\prime i}}{\partial x^{k}} \frac{\partial x^{\prime j}}{\partial x^{m}} J^{k m}(x) \nabla_{j} H^{\prime} \equiv J^{\prime i j}\left(x^{\prime}\right) \nabla_{j} H^{\prime},
$$

where $H^{\prime}\left(x^{\prime}\right)=H\left(x\left(x^{\prime}\right)\right)$ and

$$
J^{\prime i j}\left(x^{\prime}\right)=\frac{\partial x^{\prime i}}{\partial x^{k}} \frac{\partial x^{\prime j}}{\partial x^{m}} J^{k m}(x)
$$

Hence, under coordinate transformations $J$ transforms as a contravariant anti-symmetric tensor field. Evidently, the equations for $x^{\prime}$ are of the hamiltonian form with the new hamiltonian $H^{\prime}\left(x^{\prime}\right)$ if and only if

$$
\frac{\partial x^{\prime i}}{\partial x^{k}} \frac{\partial x^{\prime j}}{\partial x^{m}} J^{k m}(x)=J^{i j}\left(x^{\prime}\right) .
$$

Diffeomorphisms of the phase space which satisfy this condition are called canonical. In other words, canonical transformations do not change the form of the Poisson (tensor) bracket. An infinitesimal diffeomorphism $x^{\prime k}=x^{k}+\xi^{k}$ is generated by a vector field $\xi$. Under such a diffeomorphism the form of an arbitrary contravariant tensor $J$ varies according to (1.16),

$$
\left(\mathscr{L}_{\xi} J\right)^{i j} \equiv J^{i j}(x)-J^{\prime i j}(x)=\xi^{k} \partial_{k} J^{i j}-\partial_{k} \xi^{i} J^{k j}-\partial_{k} \xi^{j} J^{i k} .
$$

Here $\mathscr{L}_{\xi}$ is the Lie derivative of $J$ along the vector field $\xi$. It is now obvious that infinitesimal canonical transformations correspond to those $\xi$ for which $\mathscr{L}_{\xi} J=0$.

If a manifold $\mathscr{P}$ is symplectic, then canonical transformations preserve the corresponding symplectic form, that is

$$
\mathscr{L}_{\xi} \omega=0 \text {. }
$$

For this reason, these transformations are also called symplectic or symplectomorphisms.

\footnotetext{
${ }^{2}$ The general formula for the differential of a differential form of order $k$ is [2] $(k+1) d \omega\left(\xi_{0}, \xi_{1}, \ldots, \xi_{k}\right)=\sum_{i=0}^{k}(-1)^{i} \xi_{i} \omega\left(\xi_{0}, \ldots, \hat{\xi}_{i}, \ldots, \xi_{k}\right)+\sum_{0 \leq i \leq j \leq k}(-1)^{i+j} \omega\left(\left[\xi_{,} \xi_{j}\right], \xi_{0}, \ldots, \hat{\xi}_{i}, \ldots, \hat{\xi}_{j}, \ldots \xi_{k}\right)$.
} 
An important class of canonical transformations is constituted by hamiltonian vector fields. Consider a diffeomorphism generated by a hamiltonian vector field $\xi_{f}$. From the definition (1.18) of the Lie derivative we deduce that

$$
\begin{aligned}
\left(\mathscr{L}_{\xi_{f}} J\right)^{i j} & =J^{k m} \partial_{m} f \partial_{k} J^{i j}-\partial_{k}\left(J^{i m} \partial_{m} f\right) J^{k j}-\partial_{k}\left(J^{j m} \partial_{m} f\right) J^{i k} \\
& =-\partial_{m} f \sum_{(i, j, m)} J^{i k} \partial_{k} J^{j m}=0
\end{aligned}
$$

where the sum over the cyclic permutation of indices $i, j, k$ vanishes due to the Jacobi identity. The same result follows immediately from the Cartan formula

$$
\mathscr{L}_{\xi_{f}} \omega=d\left(i_{\xi_{f}} \omega\right)+i_{\xi_{f}}(d \omega)=-d^{2} f=0
$$

since $\omega$ is closed. Hence, any hamiltonian vector field generates a canonical transformation. If a Poisson manifold is not symplectic, then hamiltonian vector fields generate symplectomorphisms of the corresponding symplectic leaves.

Generally, a hamiltonian system is a triple $(\mathscr{P},\{\}, H$,$) : a phase space \mathscr{P}$, a Poisson structure $\{$,$\} and a hamiltonian H$. For any function $f$ on the phase space, evolution equation is

$$
\frac{d f}{d t}=\{H, f\}
$$

Since $\{H, H\}=0$, the hamiltonian is automatically conserved. Therefore, the motion of the system takes place on the submanifold of the phase space defined by the equation $H=E$ where $E$ is a fixed constant (energy).

\subsection{Liouville theorem}

Among a large variety of physically relevant dynamical systems, those which admit an exact solution turn out to be rather rare. Remarkably, however, for a special class of systems solutions of the corresponding Hamilton's equations can always be found by quadratures, i.e. by solving a finite number of algebraic equations and computing a finite number of definite integrals. Dynamical systems falling in this class are generally known as Liouville integrable systems because they satisfy the assumptions of the Liouville theorem. The modern formulation of this theorem and its proof is due to Arnold [3].

Arnold-Liouville theorem. Let $\mathscr{P}$ be a $2 N$-dimensional symplectic manifold. Suppose there exist $N$ functions $f_{i} \in \mathscr{F}(\mathscr{P})$ that are pairwise in involution with respect to the corresponding Poisson bracket

$$
\left\{f_{i}, f_{j}\right\}=0, \quad \forall i, j=1, \ldots, N .
$$

Consider a common level set $\mathscr{P}_{c}$ of these functions,

$$
\mathscr{P}_{c}=\left\{x \in \mathscr{P}: f_{i}(x)=c_{i}, \quad i=1, \ldots, N\right\},
$$

where $c_{i}$ are constants. Assume that functions $f_{i}$ are independent on $\mathscr{P}_{c}$, which means that the 1 -forms $d f_{i}$ are linearly independent at each point of $\mathscr{P}_{c}$. Then

1) $\mathscr{P}_{c}$ is a smooth manifold invariant under the hamiltonian flow with $H=H\left(f_{i}\right)$. 
2) If $\mathscr{P}_{c}$ is compact and connected then it is diffeomorphic to the $N$-dimensional torus

$$
\mathbb{T}^{N}=\left\{\left(\varphi_{1}, \ldots, \varphi_{N}\right) \bmod 2 \pi\right\} .
$$

3) The motion on $\mathscr{P}_{c}$ under $H$ is conditionally periodic, that is,

$$
\frac{d \varphi_{i}}{d t}=\omega_{i}(c)
$$

4) The equations of motion can be integrated by quadratures.

We only sketch the proof referring the reader to [3] for the full treatment. Consider the hamiltonian vector fields $\xi_{i}$ corresponding to the functions $f_{i}$. Since $\xi_{i} f_{j}=0$, these vector fields are tangent to $\mathscr{P}_{c}$ and their linear independence implies that they span the tangent space of $\mathscr{P}_{c}$ at any point. Taking into account that the vector fields are in involution $\left[\xi_{i}, \xi_{j}\right]=0$, we conclude on the base of the Frobenius theorem, that $\mathscr{P}_{c}$ is a maximal integral submanifold for the distribution spanned by $\xi_{i}$. Clearly, the manifold $\mathscr{P}_{c}$ is invariant under the hamiltonian flow triggered by any $H=H\left(f_{i}\right)$. Varying the constants $c_{i}$, we obtain a foliation of almost all ${ }^{3} \mathscr{P}$ into invariant submanifolds.

The main part of the proof consists in showing that whenever $\mathscr{P}_{c}$ is compact and connected, it is a torus but not, for instance, a sphere. Let $g_{i}^{t_{i}}, t_{i} \in \mathbb{R}$, be a one-parametric group of diffeomorphisms of $\mathscr{P}$ corresponding to the hamiltonian vector field $\xi_{i}$. The one-parametric groups corresponding to different vector fields commute because the vector fields commute. As a result, one can define the following action of the abelian group $\mathbb{R}^{N}=\left\{t_{1}, \ldots, t_{N}\right\}$ on $\mathscr{P}_{c}$ :

$$
g^{t}(x)=g_{1}^{t_{1}} \cdots g_{N}^{t_{N}}(x) .
$$

Since $\mathscr{P}_{c}$ is an integral manifold for the distribution spanned by $\xi_{i}$, this action is transitive and, therefore, $\mathscr{P}_{c}$ is a homogeneous space. Thus, $\mathscr{P}_{c}$ is diffeomorphic to the quotient $\mathbb{R}^{N} / \Gamma$, where $\Gamma$ is the isotropy subgroup of $\mathbb{R}^{N}$, i.e. a set of all points $t \in \mathbb{R}^{N}$ for which $g^{t}(x)=x$. The fact that the fields $\xi_{i}$ are independent at any point of $\mathscr{P}_{c}$ implies that the action (1.21) is locally free (none of the group elements has a fixed point) and, therefore, $\Gamma$ must be a discrete subgroup of $\mathbb{R}^{N}$. By assumption $\mathscr{P}_{c}$ is compact and, therefore, $\Gamma$ should be nothing else ${ }^{4}$ but an integral lattice $\mathbb{Z}^{N}$, so that $\mathscr{P}_{c}$ is diffeomorphic to $\mathbb{R}^{N} / \mathbb{Z}^{N}=\mathbb{T}^{N}$. By the standard construction of a homogeneous space as a coset, the vector fields $\xi_{i}$ are mapped by this diffeomorphism to the translation-invariant vector fields on $\mathbb{T}^{N}$. The angle variables $\left\{\varphi_{i} \bmod 2 \pi\right\}$ parametrising the torus provide a coordinate system on $\mathscr{P}_{c}$ and they can be linearly 5 expressed via $t_{1}, \ldots, t_{N}$, namely,

$$
\varphi_{i}(t)=A_{j i} t_{j}+\varphi_{i}^{(0)} \bmod 2 \pi
$$

for some constant matrix $A$ that depends on the level set $c$. Since the evolution of $\varphi_{i}$ in the direction $t_{j}$ is driven by the vector $\xi_{j}=\left\{f_{j}, \cdot\right\}$, Hamilton's equation for $\varphi_{i}$ is

$$
\frac{d \varphi_{i}}{d t_{j}}=\left.\left\{f_{j}, \varphi_{i}\right\}\right|_{\mathscr{P}_{c}}=A_{j i}
$$

${ }^{3}$ There could be values of $c_{i}$ for which the equations $f_{i}=c_{i}$ cease to be independent.

${ }^{4}$ All discrete subgroups of $\mathbb{R}^{N}$ correspond to integral lattices $\mathbb{Z}^{k}, k \leq N$.

${ }^{5}$ This follows from $\mathbb{R}^{N} / \mathbb{Z}^{N}=\mathbb{T}^{N}$. 
From here we can find evolution of angle coordinates with respect to $H=H\left(f_{i}\right)$

$$
\frac{d \varphi_{i}}{d t}=\left.\left\{H, \varphi_{i}\right\}\right|_{\mathscr{P}_{c}}=\left.\frac{\partial H}{\partial f_{j}}\left\{f_{j}, \varphi_{i}\right\}\right|_{\mathscr{P}_{c}}=\left.\frac{\partial H}{\partial f_{j}}\right|_{\mathscr{P}_{c}} A_{j i} \equiv \omega_{i}
$$

Thus, the uniform motion on the torus $\mathbb{T}^{N}$ happens according to the law $\varphi_{i}=\varphi_{i}^{0}+\omega_{i} t$ where the numbers $\omega_{i}=\omega_{i}(c)$ are called frequencies. Now consider an equation

$$
k_{1} \omega_{1}+\ldots k_{N} \omega_{N}=0
$$

where $k=\left(k_{1}, \ldots k_{N}\right)$ is a vector with integer components. If (1.22) has at least one non-zero solution, the frequency set $\left(\omega_{1}, \ldots, \omega_{N}\right)$ is called resonant, otherwise it is non-resonant. For a non-resonant set of frequencies every trajectory is dense on $\mathbb{T}^{N}$ and the corresponding motion is called conditionally periodic. Evidently, if all the frequencies are commensurable (rationally comparable), that is for any $\omega_{i}$ and $\omega_{j}$ there exist integers $m$ and $n$ such that

$$
\omega_{i} m=\omega_{j} n
$$

then the motion is periodic. This completes the discussion of the proof.

Note that the Arnold-Liouville theorem can be extended to the case when $\mathscr{P}_{c}$ is not necessarily compact. With an additional assumption that the hamiltonian vector fields $\xi_{i}$ are complete ${ }^{6}$ on $\mathscr{P}_{c}$, one can show that each connected component of $\mathscr{P}_{c}$ is diffeomorphic to $\mathbb{T}^{k} \times \mathbb{R}^{N-k}$.

Action-angle variables. The variables $f_{i}, \varphi_{j}, i, j=1, \ldots, N$ featuring in the Arnold-Liouville theorem are not in general canonical coordinates on $\mathscr{P}$. However, the latter coordinates can be constructed. First we note that in a small neighbourhood of $\mathscr{P}_{c}$ the symplectic manifold $\mathscr{P}$ is diffeomorphic to the direct product $\mathbb{D} \times \mathbb{T}^{N}$, where $\mathbb{D}$ is a small domain in $\mathbb{R}^{N}$. It turns out that in $\mathbb{D} \times \mathbb{T}^{N}$ there exist coordinates $I_{i}, \theta_{j}$, where $I_{i} \in \mathbb{D}, \theta_{j} \in \mathbb{T}^{N}$ such that all $f_{i}$ are functions of $I_{j}$ only and that the symplectic structure has the canonical form $\omega=d I_{i} \wedge d \theta_{i}$. An explicit construction of the canonical variables $I_{i}, \theta_{j}$ proceeds as follows.

It is clear that in the small neighbourhood of $\mathscr{P}_{c}$ the non-singular matrix of Poisson brackets takes the form

$$
\left(\begin{array}{ll}
\left\{f_{i}, f_{j}\right\} & \left\{f_{i}, \varphi_{j}\right\} \\
\left\{\varphi_{i}, f_{j}\right\} & \left\{\varphi_{i}, \varphi_{j}\right\}
\end{array}\right) \equiv\left(\begin{array}{cc}
0 & A_{i j} \\
-A_{j i} & B_{i j}
\end{array}\right) .
$$

The matrix $A$ is constant on $\mathscr{P}_{c}$ and therefore $A_{i j}=A_{i j}(f)$. We show that $B_{i j}$ also depends on $f_{i}$ only. Consider the Jacobi identity

$$
\left\{f_{m},\left\{\varphi_{i}, \varphi_{j}\right\}\right\}+\left\{\varphi_{i},\left\{\varphi_{j}, f_{m}\right\}\right\}+\left\{\varphi_{j},\left\{f_{m}, \varphi_{i}\right\}\right\}=0 .
$$

We have

$$
\begin{aligned}
& \left\{\varphi_{i},\left\{\varphi_{j}, f_{m}\right\}\right\}+\left\{\varphi_{j},\left\{f_{m}, \varphi_{i}\right\}\right\}=-\left\{\varphi_{i}, A_{m j}(f)\right\}+\left\{\varphi_{j}, A_{m i}(f)\right\} \\
& =-\frac{\partial A_{m j}}{\partial f_{k}}\left\{\varphi_{i}, f_{k}\right\}+\frac{\partial A_{m i}}{\partial f_{k}}\left\{\varphi_{j}, f_{k}\right\}=\frac{\partial A_{m j}}{\partial f_{k}} A_{k i}-\frac{\partial A_{m i}}{\partial f_{k}} A_{k j},
\end{aligned}
$$

${ }^{6} \mathrm{~A}$ vector field is complete if any of its flow curves exists for all values of time. 
which is the expression independent of $\varphi$. Thus, the bracket

$$
\left\{f_{m},\left\{\varphi_{i}, \varphi_{j}\right\}\right\}=\left\{f_{m}, B_{i j}\right\}=\frac{\partial B_{i j}}{\partial \varphi_{k}}\left\{f_{m}, \varphi_{k}\right\}=A_{m k} \frac{\partial B_{i j}}{\partial \varphi_{k}}
$$

is also $\varphi$-independent. Since the matrix $A$ is invertible (otherwise the Poisson bracket (1.23) would be degenerate), $\frac{\partial B_{i j}}{\partial \varphi_{k}}$ also depends only on $f$ which further implies that

$$
B_{i j}=c_{i j}^{k}(f) \varphi_{k}+g_{i j}(f) .
$$

Single-valuedness of the bracket requires that $c_{i j}^{k}=0$ (otherwise the bracket at 0 and at $2 \pi$ for any of the angles will have different values although it corresponds to the one and the same value on the torus), i.e. $B_{i j}$ is a function of $f$. One of the consequences of this fact is that the Jacobi identity (1.24) reduces to

$$
\frac{\partial A_{m j}}{\partial f_{k}} A_{k i}-\frac{\partial A_{m i}}{\partial f_{k}} A_{k j}=0 .
$$

Let us now perform the change of variables $f_{i}=f_{i}\left(I_{j}\right)$ such that $\left\{I_{i}, \varphi_{j}\right\}=\delta_{i j}$. For this we need to solve a system of equations

$$
A_{i j}=\left\{f_{i}, \varphi_{j}\right\}=\frac{\partial f_{i}}{\partial I_{k}}\left\{I_{k}, \varphi_{j}\right\}=\frac{\partial f_{i}}{\partial I_{k}} \delta_{k j}=\frac{\partial f_{i}}{\partial I_{j}} .
$$

The compatibility condition for this system of equations is

$$
\frac{\partial A_{i j}}{\partial I_{s}}=\frac{\partial f_{i}}{\partial I_{s} \partial I_{j}}=\frac{\partial f_{i}}{\partial I_{j} \partial I_{s}}=\frac{\partial A_{i s}}{\partial I_{j}} .
$$

Since $\frac{\partial A_{i j}}{\partial I_{s}}=\frac{\partial f_{k}}{\partial I_{s}} \frac{\partial A_{i j}}{\partial f_{k}}=\frac{\partial A_{i j}}{\partial f_{k}} A_{k s}$, this condition is equivalent to

$$
\frac{\partial A_{i j}}{\partial f_{k}} A_{k s}=\frac{\partial A_{i s}}{\partial f_{k}} A_{k j},
$$

which is nothing else but the Jacobi identity (1.26) for functions $f_{i}, \varphi_{j}, \varphi_{s}$.

If the variables $\varphi_{i}$ do not Poisson-commute, we should introduce new angle coordinates $\theta_{i} \bmod 2 \pi$ by making a shift $\varphi_{i}=\theta_{i}+h_{i}(I)$, where functions $h_{i}$ are determined from the condition

$$
B_{i j}=\left\{h_{i}, \theta_{j}\right\}+\left\{\theta_{i}, h_{j}\right\}=\frac{\partial h_{i}}{\partial I_{j}}-\frac{\partial h_{j}}{\partial I_{i}}
$$

The solubility condition for (1.27) is equivalent to the system of equations

$$
\frac{\partial B_{i j}}{\partial I_{k}}+\frac{\partial B_{j k}}{\partial I_{i}}+\frac{\partial B_{k i}}{\partial I_{j}}=0
$$

which is the same as

$$
\frac{\partial B_{i j}}{\partial f_{m}} A_{m k}+\frac{\partial B_{j k}}{\partial f_{m}} A_{m i}+\frac{\partial B_{k i}}{\partial f_{m}} A_{m j}=0 .
$$


Since $\left\{\left\{\varphi_{i}, \varphi_{j}\right\}, \varphi_{k}\right\}=\left\{B_{i j}, \varphi_{k}\right\}=\frac{\partial B_{i j}}{\partial f_{m}}\left\{f_{m}, \varphi_{k}\right\}=\frac{\partial B_{i j}}{\partial f_{m}} A_{m k}$, one immediately recognizes that (1.28) is just the Jacobi identity

$$
\left\{\left\{\varphi_{i}, \varphi_{j}\right\}, \varphi_{k}\right\}+\left\{\left\{\varphi_{j}, \varphi_{k}\right\}, \varphi_{i}\right\}+\left\{\left\{\varphi_{k}, \varphi_{i}\right\}, \varphi_{j}\right\}=0 .
$$

In this way we have constructed the action-angle variables $I_{i}, \varphi_{j}$ realising the canonical structure.

Note that even in the one-dimensional case action-angle variables are not uniquely defined. Action variables $I_{j}$ are defined up to additive constants and angle variables $\varphi_{j}$ can be shifted $\theta_{j} \rightarrow \theta+h_{j}(I)$ by any functions $h_{j}$ of action variables which obey the conditions $\frac{\partial h_{i}}{\partial I_{j}}-\frac{\partial h_{j}}{\partial I_{i}}=0$.

Example of an explicit construction of action-angle variables. Consider a Liouville integrable system with the phase space $\mathbb{R}^{2 N}$. According to the Liouville theorem, the motion occurs on a $N$-dimensional torus $\mathbb{T}^{N}$ being a common level of $N$ commuting integrals. Let $\gamma_{j}, 1 \leq j \leq N$, be the fundamental cycles of this torus depending continuously on the level $\left\{c_{j}\right\}$. Consider a set of equations $f_{j}(p, q)=c_{j}$ and solve it for $p_{j}: p_{j}=p_{j}(c, q)$. Introduce the so-called action variables ${ }^{7}$

$$
I_{j}(c)=\frac{1}{2 \pi} \oint_{\gamma_{j}} p_{i}(q, c) d q_{i}=\frac{1}{2 \pi} \oint_{\gamma_{j}} \alpha,
$$

where $\alpha=p_{i} d q_{i}$ is the canonical 1-form. Since $c_{j}$ are time-independent as they are values of the integrals of motion, the variables $I_{j}=I_{j}(c)$ are also time-independent. Moreover, assuming that $I_{i}$ are independent functions of $c_{j}$, the map $c_{j} \rightarrow I_{j}(c)$ given by (1.29) has an inverse. The angle variables $\theta_{j}$ are constructed by requiring that the transformation

$$
\left(p_{j}, q_{j}\right) \rightarrow\left(I_{j}, \theta_{j}\right)
$$

is canonical. To construct this canonical transformation, we will use the following generating function depending on the "old" coordinates $q$ and the "new" momenta $I$

$$
S(I, q)=\int_{q_{0}}^{q} p_{i}\left(q^{\prime}, I\right) d q_{i}^{\prime},
$$

where an integration path lies on $\mathscr{P}_{c}$. We have

$$
p_{j}=\frac{\partial S}{\partial q_{j}} \quad \rightarrow \quad p_{j}=p_{j}(I, q) .
$$

The angle variables are introduced as

$$
\theta_{j}=\frac{\partial S}{\partial I_{j}} \quad \rightarrow \quad \theta_{j}=\theta_{j}(I, q) .
$$

Thus, for the differential of $S$ we then have

$$
d S=\frac{\partial S}{\partial q_{j}} d q_{j}+\frac{\partial S}{\partial I_{j}} d I_{j}=p_{j} d q_{j}+\theta_{j} d I_{j} .
$$

Acting on this relation with $d$ and taking into account that $d^{2} S=0$, we get

$$
\omega=d p_{j} \wedge d q_{j}=d I_{j} \wedge d \theta_{j},
$$

\footnotetext{
${ }^{7}$ The physical dimension of $I_{j}$ coincide with the dimension of action that is the same as of angular momentum.
} 
which shows that $I_{i}, \theta_{j}$ are canonical variables.

A subtle point concerns a dependence of $S$ on the integration path. Consider a closed path: from $q_{0}$ to $q$ and further from $q$ to $q_{0}$. If this path is contractable, then by Stokes' theorem

$$
\Delta S=\oint_{q_{0}}^{q_{0}} \alpha=\int d \alpha=\int \omega=0 .
$$

Here the vanishing of the integral of $\omega$ is due to the fact that $\omega$ vanishes on $\mathscr{P}_{c}$

$$
\omega\left(\xi_{i}, \xi_{j}\right)=\left\{f_{i}, f_{j}\right\}=0 .
$$

If an integration path encloses a non-trivial cycle $\gamma$, the generation function undergoes a shift by an integral of $\alpha$ over this cycle

$$
\Delta_{\gamma} S=\int_{\gamma} \alpha
$$

that depends on $I_{j}$ only. As a result, going over the cycle the variables $\theta_{j}$ undergo a jump

$$
\Delta_{\gamma} \theta_{j}=\frac{\partial}{\partial I_{j}} \int_{\gamma} p_{i}(q, I) d q_{i},
$$

i.e. $\theta_{j}$ are multi-valued functions on $\mathscr{P}_{c}$. In particular, $\Delta_{\gamma_{i}} \theta_{j}=2 \pi \delta_{i j}$. This shows that $\theta_{j}$ are independent angle coordinates on the cycles. The same conclusion can be also drawn from the following consideration

$$
\oint_{\gamma_{j}} d \theta_{i}=\oint_{\gamma_{j}} d \frac{\partial S}{\partial I_{i}}=\frac{\partial}{\partial I_{i}}\left(\oint_{\gamma_{j}} d S\right)=\frac{\partial}{\partial I_{i}}\left(\oint_{\gamma_{j}} \frac{\partial S}{\partial q_{k}} d q_{k}\right)=\frac{\partial}{\partial I_{i}}\left(\oint_{\gamma_{j}} p_{k} d q_{k}\right)=2 \pi \delta_{i j},
$$

as along $\gamma_{j} \in \mathbb{T}^{N}$ the variables $I_{j}$ are constants and the function $S(I, q)$ depends on $q$ only.

In the variables $I, \theta$ the hamiltonian is a function of $I$ and the equations of motion are

$$
\dot{I}_{j}=-\frac{\partial H}{\partial \theta_{j}}=0, \quad \dot{\theta}_{j}=\frac{\partial H}{\partial I_{j}} \equiv \omega_{j}(I) .
$$

These equations are trivially solved, $I_{j}(t)=I_{j}^{0}, \theta_{j}(t)=\theta_{j}^{0}+\omega_{j}\left(I^{0}\right) t$. On the way of constructing the angle coordinates $\theta_{j}$, algebraic operations were used to find $p_{j}$ from $f_{j}(p, q)=c_{j}$ and a computation of a definite integral was implicitly done to obtain $S(I, q)$. Finally, the inverse of (1.30) was constructed by solving equations (1.32) for $q_{j}=q_{j}(I, \theta)$, which is also an algebraic operation. This way of solving a Liouville integrable system is behind the term "quadrature".

A simple example of the above construction of the action-angle variables is provided by the one-dimensional harmonic oscillator (of unit mass) which dynamics is driven by the hamiltonian

$$
H=\frac{1}{2}\left(p^{2}+\omega^{2} q^{2}\right)
$$

and the canonical bracket $\{p, q\}=1$. The reader can verify that the action-angle variables $I, \theta$ are related to $p, q$ as

$$
q=\sqrt{\frac{2 I}{\omega}} \sin \theta, \quad p=\sqrt{2 I \omega} \cos \theta .
$$




\subsection{Some examples of integrable systems}

By now a vast number of dynamical systems that fit the framework of Liouville integrability is known. Below we introduce a few such finite-dimensional systems which either were historically among the first discovered or will serve for illustrative purpose of various methods and techniques in the subsequent discussion. Our examples include spinning tops, multi-body systems of CalogeroMoser-Sutherland type and the one-dimensional bose gas.

Generalised Euler's top. The motion of rigid bodies is one of the classical problems of analytic mechanics. Since 18th century a special attention was paid to the dynamics of spinning tops, where the cases of Euler, Lagrange and Kowalevski give ubiquitous examples of completely integrable systems. Here we restrict ourselves to the discussion of the generalised Euler top. This is an $n$ dimensional rigid body without any particular symmetry that rotates in the absence of any external forces around a fixed point that we choose to coincide with its center of mass. For case $n=3$ corresponds to the conventional Euler top. To describe the dynamics, we introduce a matrix $g \in \mathrm{SO}(n)$ that connects a rotating coordinate frame rigidly fixed to the body with a stationary (inertial) frame. The lagrangian of the system is

$$
L=-\frac{1}{2} \operatorname{Tr}\left(\mathscr{I} \Omega^{2}\right), \quad \Omega=-g^{-1} \dot{g},
$$

where $\mathscr{I}$ is a symmetric positive definite matrix (inertia tensor), which we can always take diagonal. This lagrangian defines a left-invariant riemannian metric on $\mathrm{SO}(n)$ and, therefore, describes the geodesic flow on $\mathrm{SO}(n)$ corresponding to this metric. The orthogonal matrix $g$ comprises $n(n-1) / 2$ degrees of freedom which play a role of generalised coordinates. Since $g^{t} g=\mathbb{1}$, the matrix $\Omega$ is skew-symmetric: $\Omega^{t}=-\Omega$. Introducing a skew-symmetric matrix

$$
\Lambda=\frac{1}{2}(\Omega \mathscr{I}+\mathscr{I} \Omega),
$$

the equations of motion for $g$ can be written as

$$
\dot{\Lambda}=[\Omega, \Lambda]
$$

and they are known as the Euler-Arnold equations. Physically, $\Omega$ and $\Lambda$ are the angular velocity and the angular momentum in the body frame, while (1.35) are Euler's equations describing evolution of the angular momentum (or angular velocity) in the body frame. The invariance of the lagrangian under constant left shifts $g \rightarrow h g, h \in \mathrm{SO}(n)$, leads to the existence of the conserved Noether's charge $J=g \Lambda g^{-1}$, which is nothing else but the angular momentum in the stationary frame. Although the lagrangian is invariant under left shifts, the Noether charge is not, it transforms as $J \rightarrow h J h^{-1}$. The invariant integrals are constructed as $I_{k}=\operatorname{Tr} J^{k}=\operatorname{Tr} \Lambda^{k}$.

The hamiltonian description is constructed by introducing the canonical momenta ${ }^{8}$

$$
p_{j i}=\frac{\partial L}{\partial \dot{g}_{i j}}=-\frac{1}{2}\left(g^{-1} \dot{g} \mathscr{I} g^{-1}\right)_{j i}-\frac{1}{2}\left(\mathscr{I} g^{-1} \dot{g} g^{-1}\right)_{j i},
$$

In the matrix form $p=\frac{1}{2}(\Omega \mathscr{I}+\mathscr{I} \Omega) g^{-1}$, so that

$$
p g=\Lambda
$$

${ }^{8} \mathrm{By}$ definition we regard $p_{j i}$ as the canonical momentum conjugate to the coordinate $g_{i j}$. 
In deriving the canonical momentum, we have treated all the components of $g$ as independent. In the hamiltonian formulation the fact that $g$ is an orthogonal matrix can be accounted by introducing a constraint

$$
C_{1}=\mathbb{1}-g^{t} g=0 .
$$

Differentiating this constraint in time, we get $\dot{C}_{1}=\Omega^{t}+\Omega$. Thus, if we want to keep $C_{1}=0$ for all times, we have to require that $\Omega$ is a skew-symmetric matrix. Then, it follows from the definition (1.34) that $\Lambda$ is also skew-symmetric which yields due to (1.36) another hamiltonian constraint

$$
C_{2}=(p g)^{t}+p g=0 .
$$

This constraint means that not all the components of the canonical momentum are independent, rather from (1.38) together with (1.37) one gets that

$$
p^{t}=-g p g
$$

Note that the above relation also implies that $(g p)^{t}+g p=0$. No new constraints further arise; if we differentiate $C_{2}$ then the corresponding equation will be satisfied due to the equations of motion.

The hamiltonian is determined through the Legendre transform

$$
H=p_{i j} \dot{g}_{j i}-L=\operatorname{Tr}(p \dot{g})+\frac{1}{2} \operatorname{Tr}(\Lambda \Omega)=-\frac{1}{2} \operatorname{Tr}(p g \Omega),
$$

where we have used (1.36) and $\dot{g}=-g \Omega$. Further, the expression for $\Omega$ in terms of canonical variables follows from (1.34) combined with (1.36)

$$
2(p g)_{i j}=(\Omega \mathscr{I}+\mathscr{F} \Omega)_{i j}=\left(\mathscr{I}_{i}+\mathscr{I}_{j}\right) \Omega_{i j},
$$

from where we find

$$
\Omega_{i j}=\frac{2(p g)_{i j}}{\mathscr{I}_{i}+\mathscr{I}_{j}}=\frac{2 \Lambda_{i j}}{\mathscr{I}_{i}+\mathscr{I}_{j}}
$$

Thus, the positive-definite hamiltonian is the following function of the canonical coordinates and momenta

$$
H=-\frac{1}{2}(p g)_{i j} \Omega_{j i}=\sum_{i \neq j} \frac{(p g)_{i j}^{2}}{\mathscr{I}_{i}+\mathscr{I}_{j}} .
$$

Determination of the Poisson structure between the canonical variables is not straightforward. Because of constraints, it is clear that the Poisson bracket between the coordinates and momenta cannot be the canonical bracket $\left\{p_{i j}, g_{k l}\right\}=\delta_{i l} \delta_{j k}$, as the latter is incompatible with constraints (1.37) and (1.38). An educated guess for the Poisson bracket compatible with constraints is

$$
\begin{aligned}
& \left\{g_{i j}, g_{k l}\right\}=0 \\
& \left\{p_{i j}, g_{k l}\right\}=\frac{1}{2}\left(\delta_{i l} \delta_{j k}-g_{k i} g_{j l}\right) \\
& \left\{p_{i j}, p_{k l}\right\}=\frac{1}{2}\left(\delta_{i k}(g p)_{j l}-\delta_{j l}(p g)_{i k}\right)
\end{aligned}
$$


One way to derive (1.41) from the canonical Poisson bracket $\left\{p_{i j}, g_{k l}\right\}=\delta_{i l} \delta_{j k}$ is to use the Dirac bracket construction known in the theory of constrained hamiltonian systems. Another way is to note that (1.41) is equivalent to the canonical Poisson structure of the cotangent bundle $\mathrm{T}^{*} \mathrm{SO}(n)$ of the orthogonal group $\mathrm{SO}(n)$.

More generally, the cotangent bundle $\mathrm{T}^{*} G$ to a Lie group $G$ is a manifold isomorphic to the product $\mathrm{T}^{*} G \simeq G \times \mathfrak{g}^{*}$, where $\mathfrak{g}^{*}$ is the dual space to the Lie algebra $\mathfrak{g}$ of $G$. If the space $\mathfrak{g}$ is supplied with a non-degenerate bilinear form, we can use this form to identify $\mathfrak{g}^{*}$ with $\mathfrak{g}: \mathfrak{g}^{*} \simeq \mathfrak{g}$, so that the cotangent bundle is isomorphic to $G \times \mathfrak{g}$. As such, it can be parametrised by elements $(g, \ell)$, where $g \in G$ and $\ell \in \mathfrak{g}$. Matrix elements of the defining representations of $G$ in $\operatorname{GL}(n, \mathbb{C})$ and $\mathfrak{g}$ in $\operatorname{Mat}(n, \mathbb{C})$ can be regarded as coordinate functions on the cotangent bundle; we denote these coordinate functions as $g_{i j}$ and $\ell_{i j}$, respectively, where $i, j=1, \ldots, n$. Finally, the cotangent bundle of $G$ is a Poisson manifold with the following Poisson bracket which we write as the set of brackets between the coordinate functions

$$
\begin{aligned}
& \left\{g_{1}, g_{2}\right\}=0, \\
& \left\{\ell_{1}, g_{2}\right\}=g_{2} C_{12}, \\
& \left\{\ell_{1}, \ell_{2}\right\}=\left[C_{12}, \ell_{1}\right]=\frac{1}{2}\left[C_{12}, \ell_{1}-\ell_{2}\right] .
\end{aligned}
$$

Here subscript 1 and 2 stands as a concise notation for the matrix indices $i j$ and $k l$, respectively, and $C_{12} \in \mathfrak{g} \otimes \mathfrak{g}$ is the so-called split Casimir. For any $A \in \mathfrak{g}$ the spilt Casimir has the property that

$$
A_{1}=\operatorname{Tr}_{2} C_{12} A_{2},
$$

which in components means $A_{i j}=C_{i j, k l} A_{l k}$, i.e. $C$ is the identity operator in $\mathfrak{g}$.

Let us now specify the group $G$ and the Poisson structure of its cotangent bundle to the case of interest $G=\mathrm{SO}(n)$. For the orthogonal group the corresponding split Casimir is

$$
C_{i j, k l}=\frac{1}{2}\left(\delta_{i l} \delta_{j k}-\delta_{i k} \delta_{j l}\right) .
$$

It is skew-symmetric with respect to the interchange $i \leftrightarrow j$ and separately with respect to $k \leftrightarrow l$ and it fulfils (1.43) for any skew-symmetric matrix $A$. For the Poisson structure (1.42) we then find in components

$$
\begin{aligned}
& \left\{g_{i j}, g_{k l}\right\}=0, \\
& \left\{\ell_{i j}, g_{k l}\right\}=g_{k m} C_{i j, m l}=\frac{1}{2}\left(\delta_{i l} g_{k j}-\delta_{j l} g_{k i}\right), \\
& \left\{\ell_{i j}, \ell_{k l}\right\}=C_{i m, k l} \ell_{m j}-\ell_{i m} C_{m j, k l}=\frac{1}{2}\left(\delta_{i l} \ell_{k j}-\delta_{i k} \ell_{l j}-\delta_{j k} \ell_{i l}+\delta_{j l} \ell_{i k}\right) .
\end{aligned}
$$

Now one can verify that with an identification

$$
\ell=p g=\Lambda
$$

the Poisson structure (1.41) for $g$ and $p$ precisely yields the structure (1.44). To prove this result, upon evaluation of the brackets by the Leibniz rule one has to use the constraints (1.37) and (1.38). This shows that a generalised Euler's top can be understood as a dynamical system on the cotangent bundle of the orthogonal group $\mathrm{SO}(n)$. 
Formulae (1.42) render the Poisson structure of $\mathrm{T}^{*} G$ in the left parametrisation. The right parametrisation amounts to replacing $\ell$ with an element

$$
m=g \ell g^{-1} .
$$

The Poisson structure of the cotangent bundle in the right parametrisation is

$$
\begin{aligned}
\left\{g_{1}, g_{2}\right\} & =0, \\
\left\{m_{1}, g_{2}\right\} & =C_{12} g_{2}, \\
\left\{m_{1}, m_{2}\right\} & =-\frac{1}{2}\left[C_{12}, m_{1}-m_{2}\right] .
\end{aligned}
$$

Physically, $m$ coincides with the angular momentum in the stationary frame: $m=g(p g) g^{-1}=$ $g p=g \Lambda g^{-1}=J$. By using either (1.41) or (1.42), one can show that angular momenta in the moving and stationary frames Poisson commute $\left\{\ell_{i j}, m_{k l}\right\}=\left\{\Lambda_{i j}, J_{k l}\right\}=0$.

According to the last line in (1.44), the Poisson bracket between the components of $\Lambda$ is closed

$$
\left\{\Lambda_{i j}, \Lambda_{k l}\right\}=\frac{1}{2}\left(\delta_{i l} \Lambda_{k j}-\delta_{i k} \Lambda_{l j}-\delta_{k j} \Lambda_{i l}+\delta_{j l} \Lambda_{i k}\right)
$$

The matrix $\Lambda$ is skew-symmetric and the above relations coincide with the defining relations of the Lie algebra so $(n)$ for the case of general $n$. The hamiltonian can also be expressed via $\Lambda$ only

$$
H=\sum_{i \neq j} \frac{\Lambda_{i j}^{2}}{\mathscr{I}_{i}+\mathscr{I}_{j}} .
$$

The Euler equations are hamiltonian with respect to the Poisson structure (1.47) and the hamiltonian (1.48). An algebraic variety generated by the matrix elements $\Lambda_{i j}$ of a skew-symmetric matrix $\Lambda$ coincides with the dual space $\mathfrak{g}^{*}$ to the Lie algebra $\mathfrak{g}=\operatorname{so}(n)$. In opposite to the Poisson structure of the cotangent bundle, the bracket (1.47) is degenerate and $C_{k}=\operatorname{Tr} \Lambda^{2 k}$ with $k=1, \ldots,[n / 2]$ are its independent Casimir functions. As will be shown later, symplectic leaves of (1.47) coincide with orbits of the coadjoint action of $G$ in $\mathfrak{g}^{*}$. A phase trajectory of the top lies entirely on the orbit specified by constant values of $C_{k}$. For $n=3$, a coadjoint orbit, which is the phase space of the top, has dimension 2 and, therefore, having the conserved hamiltonian is enough to render Euler's top Liouville integrable. For general $n$, in addition to $C_{k}$, there are $\frac{1}{4} n(n-1)-\frac{1}{2}[n / 2]$ integrals that guarantee the Liouville integrability of the top on a regular orbit of dimension $\frac{1}{2} n(n-1)-[n / 2]$. We present these integrals in slightly after.

Calogero-Moser-Sutherland (CMS) models. Inverting the harmonic potential of the one-dimensional oscillator, one obtains a model with the hamiltonian

$$
H=\frac{p^{2}}{2 m}+\frac{\gamma^{2}}{m q^{2}} .
$$

The corresponding dynamical system can be thought of as describing radial motion of a free particle on a two-dimensional plane with fixed angular momentum $L_{\varphi}$ attributed to the coupling constant $\gamma$, the latter has the physical dimension of Planck's constant. The potential gives rise to centrifugal inverse-cube force. As any one-dimensional model with conserved energy, it can be elementary 
solved by quadratures. It is remarkable, however, that this model admits an integrable generalisation to many degrees of freedom

I.

$$
H=\frac{1}{2 m} \sum_{i=1}^{N} p_{i}^{2}+\frac{\gamma^{2}}{2 m} \sum_{i \neq j}^{N} \frac{1}{q_{i j}^{2}} .
$$

The latter model describes $N$ particles on a line interacting by the inverse-square potential. Here $q_{i j}=q_{i}-q_{j}$ is the difference between coordinates of $i$ 'th and $j$ 'th particle on a line. This mechanical system with $n$ degrees of freedom is historically tied up with names of Calogero and Moser who solved it first in the quantum and classical cases, respectively.

It has been shown by Sutherland that the model (1.49) can be further generalised to account for a periodic boundary conditions. The corresponding potential is a trigonometric generalisation of the one in (1.49) and the hamiltonian is

$$
\text { II. } \quad H=\frac{1}{2 m} \sum_{i=1}^{N} p_{i}^{2}+\frac{\gamma^{2}}{2 m} \sum_{i \neq j}^{N} \frac{1}{4 \ell^{2} \sin ^{2} \frac{1}{2 \ell} q_{i j}} \text {. }
$$

This is the Sutherland model. It can be viewed as an integrable deformation of (1.49) depending on an additional length parameter $\ell$. Particles are confined here to a ring of circumference $2 \pi \ell$, the decompactification limit $\ell \rightarrow \infty$ brings (1.50) back to the rational case (1.49). Sutherland used this model to study thermodynamical properties of quantum fluid based on (1.49). The Sutherland model has an interesting variant where the length $\ell$ is analytically continued to imaginary values $\ell \rightarrow i \ell$, giving rise to the hyperbolic model with the inverse-sinh-squared potential

$$
\text { III. } \quad H=\frac{1}{2 m} \sum_{i=1}^{N} p_{i}^{2}+\frac{\gamma^{2}}{2 m} \sum_{i \neq j}^{N} \frac{1}{4 \ell^{2} \sinh ^{2} \frac{1}{2 \ell} q_{i j}} \text {. }
$$

This time $\ell$ is naturally interpreted as an interaction length that sets the size of the region where interactions between particles are sizeable. In the limit $\ell \rightarrow \infty$ one again recovers the long-range model (1.49).

Evidently, the three models (1.49)-(1.51) are particular instances of the hamiltonian system with a pairwise potential $v(q)=v(-q)$

$$
H=\frac{1}{2 m} \sum_{i=1}^{N} p_{i}^{2}+\sum_{i<j}^{N} v\left(q_{i j}\right)
$$

One can therefore ask a question on the most general function $v(q)$ for which the model defined by the hamiltonian is integrable in the Liouville sense. The answer turns out to be

IV.

$$
v(q)=\frac{\gamma^{2}}{m} \wp(q)
$$

where $\wp(q) \equiv \wp\left(q \mid \omega_{1}, \omega_{2}\right)$ is the Weierstrass elliptic function with half-periods $\omega_{1}$ and $\omega_{2}$, where we choose $\omega_{1},-i \omega_{2}$ to be any positive numbers, possibly infinite. ${ }^{9}$ This potential defines an elliptic model from which the previous models follow as degenerate cases when one or both periods become infinite. Specifically, we have

\footnotetext{
${ }^{9}$ The $\wp$-function is homogeneous $\wp\left(\lambda q \mid \lambda \omega_{1}, \lambda \omega_{2}\right)=\lambda^{-2} \wp\left(q \mid \omega_{1}, \omega_{2}\right)$. With the assumption that $\omega_{1}, \omega_{2}$ has the physical dimension of length, this property allows one to use in $\wp(q)$ the dimensional coordinate $q$.
} 
Rational case: $\omega_{1}=\infty, \omega_{2}=i \infty$,

$$
\wp(q) \rightarrow \frac{1}{q^{2}}
$$

Hyperbolic case: $\omega_{1}=\infty, \omega_{2}=i \pi \ell$,

$$
\wp(q) \rightarrow \frac{1}{4 \ell^{2} \sinh ^{2} \frac{q}{2 \ell}}+\frac{1}{12 \ell^{2}} .
$$

Trigonometric case: $\omega_{1}=\pi \ell, \omega_{2}=i \infty$,

$$
\wp(q) \rightarrow \frac{1}{4 \ell^{2} \sin ^{2} \frac{q}{2 \ell}}-\frac{1}{12 \ell^{2}} .
$$

The models with rational, trigonometric, hyperbolic and elliptic potentials are marked as I, II, III and IV, respectively. In the following we abbreviate the dynamical systems I - IV as the CMS (Calogero-Moser-Sutherland) models. ${ }^{10}$ These CMS models are related to the root system of the Lie algebra $A_{N-1}$ and can be generalised to other root systems [4].

Bose gas with delta-interaction. The so-called delta-interaction model is defined by the hamiltonian

$$
H=\frac{1}{2 m} \sum_{i=1}^{N} p_{i}^{2}+\kappa \sum_{i<j} \delta\left(q_{i}-q_{j}\right),
$$

where $\kappa$ is a real coupling constant. For $\kappa>0$ the interaction is repulsive and for $\kappa<0$ it is attractive. A solution of the corresponding quantum-mechanical problem for the repulsive case was first obtained in the case of bosons by Lieb and Liniger, while the general case of distinguishable particles was solved by C.N. Yang. Expanded in many directions, this model serves as a prototype example of applications of the Bethe Ansatz techniques.

\subsection{Lax representation and classical $r$-matrix}

Lax representation. Let $L$ and $M$ be two square matrices whose entries are functions on a phase space. Consider the following matrix equation

$$
\dot{L}=[M, L],
$$

where as usual dot stands for the time derivative. If equation (1.55) is identically satisfied as a consequence of hamiltonian equations for a given dynamical system, then this dynamical system is said to admit a Lax representation (1.55) with $L$ being the corresponding Lax matrix. Such a pair of matrices $L$ and $M$ is often referred to as Lax pair.

The importance of the Lax representation is that, once found, it allows for a simple and universal construction of an extended set of conserved quantities as spectral invariants of the corresponding Lax matrix. Indeed, consider

$$
H_{k}=\operatorname{Tr} L^{k}
$$

\footnotetext{
${ }^{10} \mathrm{We}$ follow the classification of the CMS systems by [4] but for our presentation purposes interchanged II $\leftrightarrow$ III.
} 
for $k \in \mathbb{Z}$. We have

$$
\dot{H}_{k}=k \operatorname{Tr}\left(L^{k-1} \dot{L}\right)=k \operatorname{Tr}\left(L^{k-1}[M, L]\right)=\operatorname{Tr}\left[M, L^{k}\right]=0,
$$

i.e. the $H_{k}$ are time-independent as a consequence of the hamiltonian equations implying (1.55). In fact, the matrix equation (1.55) can be readily solved as

$$
L(t)=g(t) L(0) g(t)^{-1},
$$

where the invertible matrix $g(t)$ is determined from the equation

$$
M(t)=\dot{g} g^{-1} .
$$

By Newton's identities, integrals $H_{k}$ are functions of the eigenvalues of the matrix $L$ and vice versa. Since the eigenvalues of $L$ are preserved in time, evolution of such a dynamical system is called isospectral.

It should be emphasised that a Lax pair, if it exists, is not uniquely defined. First, the one and the same dynamical system might admit Lax pairs represented by $n \times n$ matrices of different size $n$. Second, there is a freedom related to transformations of the type

$$
L^{\prime}=g L g^{-1}, \quad M^{\prime}=g M g^{-1}+\dot{g} g^{-1},
$$

where $g$ is an arbitrary invertible matrix possibly depending on dynamical variables. If here $L, M$ is a Lax pair, then $L^{\prime}, M^{\prime}$ is another one for the same dynamical system. Indeed,

$$
\dot{L}^{\prime}=\dot{g} L g^{-1}+g[M, L] g^{-1}-g L g^{-1} \dot{g} g^{-1}=\left[g M g^{-1}+\dot{g} g^{-1}, g L g^{-1}\right] \equiv\left[M^{\prime}, L^{\prime}\right] .
$$

Note that $M$ undergoes a gauge-type transformation. Lastly, for a fixed $L$ shifting $M$ by any polynomial of $L$ will not influence the Lax equation (1.55).

We illustrate the concept of Lax representation on the example of the CMS models I, II and III.

I. Lax representation for the rational CMS model can be chosen as

$$
L=\sum_{i=1}^{N} p_{i} E_{i i}-i \gamma \sum_{i \neq j}^{N} \frac{1}{q_{i j}} E_{i j}, \quad M=i \gamma \sum_{i \neq j}^{N} \frac{1}{q_{i j}^{2}}\left(E_{i i}-E_{i j}\right) .
$$

Using the canonical structure (1.7) and the hamiltonian ${ }^{11}$ (1.49), one can verify the validity of the Lax representation, namely, that

$$
\dot{L}=\{H, L\}=[M, L] .
$$

The first two spectral invariants of $L$ produce the total momentum $P$ and the Hamiltonian $2 \mathrm{H} \equiv \mathrm{H}_{2}$. Starting from $\operatorname{Tr} L^{3}$ one obtaines new integrals of motion that cannot be expressed via $P$ and $H$.

\footnotetext{
${ }^{11}$ For simplicity we put in this section $m=1$ and $\ell=1$.
} 
More explicitly,

$$
\begin{aligned}
& H_{1}=\operatorname{Tr} L=\sum_{i} p_{i} \\
& H_{2}=\operatorname{Tr} L^{2}=\sum_{i} p_{i}^{2}+\gamma^{2} \sum_{i \neq j} \frac{1}{q_{i j}^{2}} \\
& H_{3}=\operatorname{Tr} L^{3}=\sum_{i} p_{i}^{3}+3 \gamma^{2} \sum_{i \neq j} \frac{p_{i}}{q_{i j}^{2}} \\
& H_{4}=\operatorname{Tr} L^{4}=\sum_{i} p_{i}^{4}+4 \gamma^{2} \sum_{i \neq j} \frac{p_{i}^{2}+\frac{1}{2} p_{i} p_{j}}{q_{i j}^{2}}+\gamma^{4} \sum_{i \neq j} \frac{1}{q_{i j}^{4}}+2 \gamma^{4} \sum_{i \neq j \neq k} \frac{1}{q_{i j}^{2} q_{j k}^{2}}
\end{aligned}
$$

As $\gamma \rightarrow 0$ the integrals behave as $H_{k} \sim \sum_{j} p_{j}^{k}$, which gives a reason to call the basis of conserved quantities constituted by these integrals the power sum basis.

The integrals $H_{k}$ have the distinguished feature of being local. This means that in the asymptotic limit of large time, they take an additive form with respect to the number of particles

$$
H_{k} \sim \sum_{j} p_{j}^{k},
$$

where $p_{j}$ is an asymptotic momentum of $j$ 's particle. Exhibiting a commutative family of operators is not exciting by itself, but the fact that these operators are local is truly remarkable. For instance, powers of the hamiltonian, although commuting, are not local operators. Thus, the existence of local commuting operators adds to the notion of (quantum) integrability.

II. Lax representation for the trigonometric CMS model. It is convenient to introduce $Q_{i}=e^{i q_{i}}$. In terms of this variable the Lax pair reads

$$
L=\sum_{i=1}^{N} p_{i} E_{i i}+\gamma \sum_{i \neq j}^{N} \frac{Q_{j}}{Q_{i j}} E_{i j}, \quad M=-i \gamma \sum_{i \neq j}^{N} \frac{Q_{i} Q_{j}}{Q_{i j}^{2}}\left(E_{i i}-E_{i j}\right) .
$$

One can verify that (1.58) holds for the canonical structure (1.7) and the hamiltonian (1.50). In terms of $Q$-variables the hamiltonian is

$$
H=\frac{1}{2} \sum_{i} p_{i}^{2}-\frac{\gamma^{2}}{2} \sum_{i \neq j} \frac{Q_{i} Q_{j}}{Q_{i j}^{2}}
$$

Computing the spectral invariants of $L$, we generate the conservation laws for this model

$$
\begin{aligned}
& H_{1}=\operatorname{Tr} L=\sum_{i} p_{i} \\
& H_{2}=\operatorname{Tr} L^{2}=\sum_{i} p_{i}^{2}-\gamma^{2} \sum_{i \neq j} \frac{Q_{i} Q_{j}}{Q_{i j}^{2}} \\
& H_{3}=\operatorname{Tr} L^{3}=\sum_{i} p_{i}^{3}-3 \gamma^{2} \sum_{i \neq j} \frac{Q_{i} Q_{j}}{Q_{i j}^{2}} p_{i} \\
& H_{4}=\operatorname{Tr} L^{4}=\sum_{i} p_{i}^{4}-\gamma^{2} \sum_{i \neq j} \frac{Q_{i} Q_{j}}{Q_{i j}^{2}}\left(4 p_{i}^{2}+2 p_{i} p_{j}\right)+\gamma^{4} \sum_{i \neq j} \frac{Q_{i}^{2} Q_{j}^{2}}{Q_{i j}^{4}}+2 \gamma^{4} \sum_{i \neq j \neq k} \frac{Q_{i} Q_{j}^{2} Q_{k}}{Q_{i j}^{2} Q_{j k}^{2}}
\end{aligned}
$$


III. Lax representation for the hyperbolic CMS model. This time we introduce $Q_{i}=e^{q_{i}}$. The Lax pair reads

$$
L=\sum_{i=1}^{N} p_{i} E_{i i}-i \gamma \sum_{i \neq j}^{N} \frac{Q_{j}}{Q_{i j}} E_{i j}, \quad M=i \gamma \sum_{i \neq j}^{N} \frac{Q_{i} Q_{j}}{Q_{i j}^{2}}\left(E_{i i}-E_{i j}\right) .
$$

Again, (1.58) holds for the canonical structure (1.7) and the hamiltonian (1.51), the latter takes in terms of $Q$-variables the form

$$
H=\frac{1}{2} \sum_{i} p_{i}^{2}+\frac{\gamma^{2}}{2} \sum_{i \neq j} \frac{Q_{i} Q_{j}}{Q_{i j}^{2}}
$$

Computing the spectral invariants of $L$, we generate the conservation laws for this model

$$
\begin{aligned}
& H_{1}=\operatorname{Tr} L=\sum_{i} p_{i} \\
& H_{2}=\operatorname{Tr} L^{2}=\sum_{i} p_{i}^{2}+\gamma^{2} \sum_{i \neq j} \frac{Q_{i} Q_{j}}{Q_{i j}^{2}} \\
& H_{3}=\operatorname{Tr} L^{3}=\sum_{i} p_{i}^{3}+3 \gamma^{2} \sum_{i \neq j} \frac{Q_{i} Q_{j}}{Q_{i j}^{2}} p_{i} \\
& H_{4}=\operatorname{Tr} L^{4}=\sum_{i} p_{i}^{4}+\gamma^{2} \sum_{i \neq j} \frac{Q_{i} Q_{j}}{Q_{i j}^{2}}\left(4 p_{i}^{2}+2 p_{i} p_{j}\right)+\gamma^{4} \sum_{i \neq j} \frac{Q_{i}^{2} Q_{j}^{2}}{Q_{i j}^{4}}+2 \gamma^{4} \sum_{i \neq j \neq k} \frac{Q_{i} Q_{j}^{2} Q_{k}}{Q_{i j}^{2} Q_{j k}^{2}}
\end{aligned}
$$

The formulae for integrals of the trigonometric and hyperbolic models share the features of their rational cousins. Restoring the length parameter $\ell$ by rescaling $q_{i} \rightarrow q_{i} / \ell p_{i} \rightarrow \ell p_{i}$, which is a canonical transformation, followed by $L \rightarrow L / \ell$ and $M \rightarrow M / \ell^{2}$, one can see that in the limit $\ell \rightarrow \infty$ the Lax pairs for both models II and III go into the Lax pair of the model I, and the same is for integrals of motion.

Babelon-Viallet theorem and dynamical r-matrix. The Lax representation makes no reference to a Poisson structure. Spectral invariants of the Lax matrix are integrals of motion but without specifying this structure it is impossible to conclude anything about their involutive property.

A relation of integrals to the underlying Poisson structure gets established due to the BabelonViallet theorem [5], see also [6]. According to this theorem, having the involutive property of the eigenvalues of $L \in \operatorname{Mat}_{n}(\mathbb{C})$ is equivalent to the existence of a function $r$ on the phase space with values in $\operatorname{Mat}_{n}(\mathbb{C})^{\otimes 2}$ such that the Poisson bracket between the entries of $L$ is

$$
\left\{L_{1}, L_{2}\right\}=\left[r_{12}, L_{1}\right]-\left[r_{21}, L_{2}\right] .
$$

As an explicit matrix, $r_{12} \equiv\left(r_{i j, k l}\right)$, where $i, j=1, \ldots, n$ correspond to the first matrix space and $k, l=1, \ldots, n$ to the second one. The matrices on the right hand side of (1.64) are multiplied according to the standard rule of matrix multiplication. Thus, being written in components, formula (1.64) looks like

$$
\left\{L_{i j}, L_{k l}\right\}=r_{i s, k l} L_{s j}-L_{i s} r_{s j, k l}-r_{k s, i j} L_{s l}+L_{k s} r_{s l, i j}, \quad \forall i, j, k, l \in 1, \ldots, n,
$$


where we have separated the indices belonging two different matrix spaces of $r_{12}$ by comma. Clearly, the use of the concise notation as in (1.64) saves a sufficient amount of work and space.

The matrix $r$ is called dynamical $r$-matrix, which reflects the possibility for this matrix to depend on the phase space variables. Note that the bracket (1.64) is manifestly skew-symmetric. To obtain (1.64), we assume that $L$ is diagonalisable,

$$
L=S \Lambda S^{-1},
$$

where $\Lambda$ is a diagonal matrix whose entries $\Lambda_{i}$ are prospective integrals of motion. Assuming that the phase space is equipped with a Poisson structure such that $\left\{\Lambda_{i}, \Lambda_{j}\right\}=0$ for any $i, j$, we compute

$$
\begin{aligned}
\left\{L_{1}, L_{2}\right\}=\left\{S_{1} \Lambda_{1} S_{1}^{-1}, S_{2} \Lambda_{2} S_{2}^{-1}\right\} & =k_{12} L_{1} L_{2}+L_{1} L_{2} k_{12}-L_{1} k_{12} L_{2}-L_{2} k_{12} L_{1} \\
& -q_{21} L_{2}+q_{12} L_{1}-L_{1} q_{12}+L_{2} q_{21},
\end{aligned}
$$

where we introduced the notation

$$
k_{12}=\left\{S_{1}, S_{2}\right\} S_{1}^{-1} S_{2}^{-1}, \quad q_{12}=S_{2}\left\{S_{1}, \Lambda_{2}\right\} S_{1}^{-1} S_{2}^{-1}, \quad q_{21}=S_{1}\left\{S_{2}, \Lambda_{1}\right\} S_{1}^{-1} S_{2}^{-1} .
$$

From the explicit form of $k_{12}$ one sees that $k_{21}=-k_{12}$. This allows one to further rearrange the bracket as

$$
\begin{aligned}
\left\{L_{1}, L_{2}\right\} & =\left[k_{12} L_{2}-L_{2} k_{12}, L_{1}\right]+\left[q_{12}, L_{1}\right]-\left[q_{21}, L_{2}\right] \\
& =\frac{1}{2}\left[\left[k_{12}, L_{2}\right], L_{1}\right]-\frac{1}{2}\left[\left[k_{21}, L_{1}\right], L_{2}\right]+\left[q_{12}, L_{1}\right]-\left[q_{21}, L_{2}\right] .
\end{aligned}
$$

The last expression has precisely the form (1.64), where the corresponding $r$-matrix is

$$
r_{12}=q_{12}+\frac{1}{2}\left[k_{12}, L_{2}\right] .
$$

Note that $r_{12}$ is not assumed to have any specific symmetry properties. Also, it is not uniquely defined: one can readily see that a shift $r_{12} \rightarrow r_{12}+\left[\sigma_{12}, L_{2}\right]$, where $\sigma_{12}=\sigma_{21}$, does not influence the right hand side of (1.64). Also, the bracket (1.64) does not change its form under symmetry transformations (1.56), although the $r$-matrix does.

Concerning the Jacobi identity for (1.64), it yields the following constraint on the $r$-matrix

$$
\left[L_{1},\left[r_{12}, r_{13}\right]+\left[r_{12}, r_{23}\right]+\left[r_{32}, r_{13}\right]+\left\{L_{2}, r_{13}\right\}-\left\{L_{3}, r_{12}\right\}\right]+\text { cycl. perm }=0 .
$$

In the case when $r$ is independent of the dynamical variables, the last equation simplifies to

$$
\left[L_{1},\left[r_{12}, r_{13}\right]+\left[r_{12}, r_{23}\right]+\left[r_{32}, r_{13}\right]\right]+\text { cycl. perm }=0 .
$$

In particular, the Jacobi identity will be satisfied if $r$ obeys the following equation

$$
\left[r_{12}, r_{13}\right]+\left[r_{12}, r_{23}\right]+\left[r_{32}, r_{13}\right]=0 .
$$

Another important point about the Poisson structure (1.64) is that it yields the Lax representation for evolution equations driven by any of the hamiltonians $H_{k}=\operatorname{Tr} L^{k}, k \in \mathbb{Z}$. Indeed, from (1.64) one gets

$$
\frac{d L}{d t_{k}}=\left\{H_{k}, L\right\}=\left[M_{k}, L\right]
$$


where $M_{k}=-k \operatorname{Tr}_{1}\left(r_{21} L_{1}^{k-1}\right)$ and $t_{k}$ is the time evolution parameter along the hamiltonian flow triggered by $H_{k}$.

Lax representation with spectral parameter. The number of integrals constructed as eigenvalues of the Lax matrix $L$ cannot exceed the rank of $L$. For systems with a substantially large number of degrees of freedom and with a Lax representation by low-rank matrices, the Liouville integrability is not guaranteed by the Lax method. For instance, for the generalised Euler top, we can immediately identify the Lax representation by taking $L=\Lambda$ and $M=\Omega$, see (1.35). However, the spectral invariants $\operatorname{Tr} L^{k}$ yield only the Casimir functions and are not enough to declare the model to be integrable. Remarkably, there is a way to extend the original approach based on (1.55) such that it will produce enough integrals for Liouville integrability.

Introduce a variable $\lambda \in \mathbb{C}$, called the spectral parameter, and consider two square matrices $L(\lambda)$ and $M(\lambda)$ that are functions of the phase space variables and $\lambda$. A given dynamical system admits a Lax representation with the spectral parameter, if there exist $L(\lambda)$ and $M(\lambda)$ such that the matrix equation

$$
\dot{L}(\lambda)=[M(\lambda), L(\lambda)]
$$

is satisfied for any value of $\lambda$ as the consequence of the equations of motion for this system. For a system which admits a Lax representation with the spectral parameter, the quantities

$$
I_{k}(\lambda)=\operatorname{Tr} L^{k}(\lambda)
$$

are conserved for any $\lambda$. Assuming $I_{k}(\lambda)$ are rational functions of $\lambda$, the coefficients of their Laurent expansion around each pole are integrals of motion. The determinant $\operatorname{det}(L(\lambda)-\zeta \mathbb{1})$, which generates $I_{k}(\lambda)$ in the power series expansion over the parameter $\zeta$, defines the classical spectral curve

$$
\operatorname{det}(L(\lambda)-\zeta \mathbb{1})=0, \quad \zeta, \lambda \in \mathbb{C} .
$$

This procedure yields an extended set of integrals. Their involutive property can be established with a generalisation of the Babelon-Viallet theorem: the spectral invariants of $L(\lambda)$ will Poisson commute at different values of $\lambda$, iff there exists a dynamical $r$-matrix $r(\lambda, \mu)$ such that the Poisson bracket between the components of $L(\lambda)$ and $L(\mu)$ is of the form

$$
\left\{L_{1}(\lambda), L_{2}(\mu)\right\}=\left[r_{12}(\lambda, \mu), L_{1}(\lambda)\right]-\left[r_{21}(\mu, \lambda), L_{2}(\mu)\right] .
$$

As an example, consider Euler's top. The spectral dependent Lax representation is given by Manakov's pair

$$
L(\lambda)=\mathscr{F}^{2}+\frac{1}{\lambda} \Lambda, \quad M(\lambda)=2 \lambda \mathscr{F}+\Omega .
$$

To check (1.67), consider

$$
\frac{1}{\lambda} \dot{\Lambda}=[M(\lambda), L(\lambda)]=\left[\Omega, \mathscr{J}^{2}\right]+2[\mathscr{F}, \Lambda]+\frac{1}{\lambda}[\Omega, \Lambda] .
$$

Vanishing of the constant term here is automatic due to the definition (1.34) of $\Lambda$, while vanishing of $1 / \lambda$-terms is equivalent to the Euler equations. Further, by applying the Poisson brackets (1.47), 
we compute the left hand side of (1.70) and find that it can be written in the $r$-matrix form with the following non-dynamical $r$-matrix

$$
r(\lambda, \mu)=-\frac{1}{2} \sum_{i j} \frac{E_{i j} \otimes E_{j i}}{\lambda-\mu}-\frac{1}{2} \sum_{i j} \frac{E_{i j} \otimes E_{i j}}{\lambda+\mu} .
$$

It is convenient to define integrals polynomial in $\lambda$

$$
I_{k}(\lambda)=\lambda^{k} \operatorname{Tr} L(\lambda)^{k}=\operatorname{Tr}\left(\Lambda+\lambda \mathscr{I}^{2}\right)^{k}=\sum_{s=0}^{k} I_{k, s} \lambda^{s},
$$

where $k=2, \ldots, n$. Coefficients $I_{k, s}$ are independent integrals and the existence of the $r$-matrix guarantees that they are in involution. Since $\Lambda$ is skew-symmetric and $\mathscr{g}^{2}$ is symmetric, $I_{k, s}$ is non-zero only if $s$ has the same parity as $k$. This observation allows one to compute the total number $\mathscr{N}(n)$ of integrals

$$
\mathscr{N}(n)=\sum_{k=2}^{n}\left[\frac{k}{2}\right]=\frac{1}{2}\left[\frac{n}{2}\right]+\frac{n(n-1)}{4},
$$

from which [ $n / 2]$ integrals $I_{k, 0}$ with $k$ even are Casimirs of (1.47). The number $\mathscr{N}(n)-[n / 2]$ of the remaining integrals is exactly half the dimension of a regular coadjoint orbit of $\mathrm{SO}(n)$.

\section{Phase spaces with symmetries and reduction}

Here we describe a way of constructing integrable models by using the method of hamiltonian reduction. This method is based on the Marsden-Weinstein theorem which allows one to construct a symplectic manifold from another one by reducing the latter over its symmetry. We start from a general definition of the Lie-Poisson structure, one example of which has been already accounted in the theory of Euler's top.

\subsection{Lie-Poisson structure and coadjoint orbits}

Let $G$ be a Lie group and $\mathfrak{g}$ be its Lie algebra. Let also $\mathfrak{g}^{*}$ be the dual of $\mathfrak{g}$ with the natural pairing $\langle\cdot, \cdot\rangle$ between $\mathfrak{g}$ and $\mathfrak{g}^{*}$. Consider the algebra of smooth functions $\mathscr{F}\left(\mathfrak{g}^{*}\right)$ on $\mathfrak{g}^{*}$. If $f \in \mathscr{F}\left(\mathfrak{g}^{*}\right)$ then its gradient $\nabla f \in \mathfrak{g}$ is defined as

$$
\langle m, \nabla f(\ell)\rangle=\lim _{t \rightarrow 0} \frac{f(\ell+t m)-f(\ell)}{t}, \quad \ell, m \in \mathfrak{g}^{*},
$$

The Kirillov-Kostant Poisson bracket is defined as

$$
\{f, h\}(\ell)=\langle\ell,[\nabla f(\ell), \nabla h(\ell)]\rangle
$$

and it supplies the dual space to the Lie algebra with the structure of a Poisson manifold. One often refers to (2.1) as to the Lie-Poisson structure. Let us fix a basis $\left\{e_{i}\right\}$ in $\mathfrak{g}$, so that

$$
\left[e_{i}, e_{j}\right]=f_{i j}^{k} e_{k}
$$


where $f_{i j}^{k}$ are structure constants. Denote by $e^{i}$ a dual basis in $\mathfrak{g}^{*}$ defined as $\left\langle e^{i}, e_{j}\right\rangle=\delta_{j}^{i}$. Then $\ell=\ell_{i} e^{i}$ and $\ell_{i}$ are coordinates on $\mathfrak{g}^{*}$. In these coordinates

$$
\nabla f=e_{i} \frac{\partial f}{\partial \ell_{i}},
$$

so that $\nabla \ell_{i}=e_{i}$. Therefore, the bracket between the coordinates is

$$
\left\{\ell_{i}, \ell_{j}\right\}=\left\langle\ell,\left[e_{i}, e_{j}\right]\right\rangle=f_{i j}^{k} \ell_{k} .
$$

It is clear that the Kirillov-Kostant bracket is degenerate as is seen, for instance, from the vanishing of the corresponding Poisson tensor at $\ell=0$. There is a geometric characterisation of symplectic leaves of the bracket (2.1) as orbits of the coadjoint representation of $G$ [7]. Below we recall the corresponding construction.

Coadjoint orbits. Denote by $\operatorname{Ad}_{g}$ and $\operatorname{ad}_{X}$ the adjoint representations of $G$ and $\mathfrak{g}$, where $g \in G$ and $X \in \mathfrak{g}$. Assuming $G$ is a matrix Lie group, one has

$$
\operatorname{Ad}_{g} X=g X g^{-1}, \quad \operatorname{ad}_{X} Y=[X, Y], \quad Y \in \mathfrak{g} .
$$

Then the coadjoint action (representation) of $G$ in the dual space $\mathfrak{g}^{*}$ is defined as

$$
\operatorname{Ad}_{g}^{*} \ell(X)=\ell\left(\operatorname{Ad}_{g^{-1}} X\right)=\ell\left(g^{-1} X g\right),
$$

for any $X \in \mathfrak{g}$. The derivative map of this action at the group unity $g=e$ defines the coadjoint action (representation) of $\mathfrak{g}$ in $\mathfrak{g}^{*}$ :

$$
\operatorname{ad}_{X}^{*} \ell=\left.\frac{d}{d t} \operatorname{Ad}_{e^{t X}}^{*} \ell\right|_{t=0}
$$

so that

$$
\operatorname{ad}_{X}^{*} \ell(Y)=-\ell\left(\operatorname{ad}_{X} Y\right)=-\ell([X, Y])
$$

Under the coadjoint action the space $\mathfrak{g}^{*}$ splits into orbits. Consider an orbit

$$
\mathscr{O}_{n}=\left\{\operatorname{Ad}_{g}^{*} n, g \in G\right\}
$$

that passes through a point $n \in \mathfrak{g}^{*}$ and denote by $G_{n} \subset G$ the stabiliser (stability group) of this point. Evidently, the orbit can be modelled as a homogenous space $G / G_{n} \approx \mathscr{O}_{n}$. Let us show that the tangent space to $\mathscr{O}_{n}$ at $n$ is then naturally identified with the factor-space $\mathfrak{g} / \mathfrak{g}_{n}$, where $\mathfrak{g}_{n}$ is the Lie algebra of $G_{n}$

$$
\mathfrak{g}_{n}=\left\{X \in \mathfrak{g}: \operatorname{ad}_{X}^{*} n=0\right\} .
$$

Any element $X \in \mathfrak{g}$ gives rise to a vector field on $\mathfrak{g}^{*}$ tangent to the orbits of the coadjoint action. For $f \in \mathscr{F}\left(\mathfrak{g}^{*}\right)$ this vector field is defined as

$$
\xi_{X} f(\ell)=\left.\frac{d}{d t} f\left(\operatorname{Ad}_{e^{-t X}}^{*} \ell\right)\right|_{t=0}=-\left\langle\operatorname{ad}_{X}^{*} \ell, \nabla f(\ell)\right\rangle .
$$


From here we find that

$$
\xi_{X}=-\left\langle\operatorname{ad}_{X}^{*} \ell, e_{i}\right\rangle \frac{\partial}{\partial \ell_{i}}=\left\langle\ell,\left[X, e_{i}\right]\right\rangle \frac{\partial}{\partial \ell_{i}}=\left\langle e^{j},\left[X, e_{i}\right]\right\rangle \ell_{j} \frac{\partial}{\partial \ell_{i}} .
$$

These fields satisfy the relation

$$
\left[\xi_{X}, \xi_{Y}\right]=\xi_{[X, Y]}
$$

implying that the map $X \rightarrow \xi_{X}$ is a homomorphism $\mathfrak{g} \rightarrow \mathfrak{X}\left(\mathfrak{g}^{*}\right)$. As follows from (2.5), for $X \in \mathfrak{g}_{n}$ the field $\xi_{X}$ vanishes at the point $n$ and vice versa. The tangent space to $\mathscr{O}_{n}$ at $n$ is spanned by non-vanishing vector fields and is, therefore, isomorphic to $\mathfrak{g} / \mathfrak{g}_{n}$.

The fields $\xi_{X}$ are hamiltonian, they are generated by the following linear functions of $\ell$

$$
f_{X}(\ell)=\langle\ell, X\rangle .
$$

Indeed, $\nabla f_{X}(\ell)=X$ and, therefore,

$$
\left\{f_{X}, h\right\}(\ell)=\langle\ell,[X, \nabla h(\ell)]\rangle=-\left\langle\operatorname{ad}_{X}^{*} \ell, \nabla h(\ell)\right\rangle=\xi_{X} h(\ell), \quad \forall h \in \mathscr{F}\left(\mathfrak{g}^{*}\right) .
$$

$G$-invariant symplectic structure on coadjoint orbits. According to (1.14), one can define on $\mathscr{O}_{n}$ a closed 2-form

$$
\omega_{\ell}\left(\xi_{X}, \xi_{Y}\right)=\left\{f_{X}, f_{Y}\right\}(\ell)=\langle\ell,[X, Y]\rangle=f_{[X, Y]}(\ell), \quad \ell \in \mathscr{O}_{n}
$$

where $\xi_{X}$ and $\xi_{Y}$ are hamiltonian vector fields (2.6) tangent to the orbit at the point $\ell$. The fact that $\omega$ is $G$-invariant can be demonstrated in different ways. One way is to note that the fields $\xi_{X}$ representing the group action on $\mathscr{F}\left(\mathfrak{g}^{*}\right)$ are hamiltonian and, according to the general theory, must preserve the Poisson bracket and the 2-form (79). Another way to be now explained appeals to a direct computation. The group acts on points of the orbit by $\ell \rightarrow \ell^{\prime}=\operatorname{Ad}_{g}^{*} \ell$. Under this action the vector fields transform: $\xi_{X} \rightarrow \xi_{X}^{\prime}$. For a fixed $g \in G$ the point $\ell^{\prime}$ has coordinates $\ell_{j}^{\prime}=\left\langle\operatorname{Ad}_{g}^{*} \ell, e_{j}\right\rangle=\left\langle\ell, \operatorname{Ad}_{g^{-1}} e_{j}\right\rangle$, so that the Jacobi matrix is

$$
\frac{\partial \ell_{i}^{\prime}}{\partial \ell_{j}}=\frac{\partial \ell_{k}}{\partial \ell_{j}}\left\langle e^{k}, \operatorname{Ad}_{g^{-1}} e_{i}\right\rangle=\left\langle e^{j}, \operatorname{Ad}_{g^{-1}} e_{i}\right\rangle
$$

The field $\xi_{X}^{\prime}$ can be then found by using the standard transformation rule for vector fields

$$
\xi_{X}^{\prime}\left(\ell^{\prime}\right)=\left\langle\ell,\left[X, e_{j}\right]\right\rangle \frac{\partial \ell_{i}^{\prime}}{\partial \ell_{j}} \frac{\partial}{\partial \ell_{i}^{\prime}}=\left\langle\ell,\left[X, e_{j}\right]\right\rangle\left\langle e^{j}, \operatorname{Ad}_{g^{-1}} e_{i}\right\rangle \frac{\partial}{\partial \ell_{i}^{\prime}}=\left\langle\ell,\left[X, \operatorname{Ad}_{g^{-1}} e_{i}\right]\right\rangle \frac{\partial}{\partial \ell_{i}^{\prime}},
$$

where we have used that $\left|e_{j}\right\rangle\left\langle e^{j}\right|$ is the identity operator on $\mathfrak{g}$. Therefore,

$$
\xi_{X}^{\prime}\left(\ell^{\prime}\right)=\left\langle\ell^{\prime},\left[\operatorname{Ad}_{g} X, e_{i}\right]\right\rangle \frac{\partial}{\partial \ell_{i}^{\prime}}=\xi_{\operatorname{Ad}_{g} X}\left(\ell^{\prime}\right),
$$

where in the last step we used the comparison with (2.6). It remains to evaluate

$$
\omega_{\ell^{\prime}}\left(\xi_{X}^{\prime}, \xi_{Y}^{\prime}\right)=\omega_{\ell^{\prime}}\left(\xi_{\operatorname{Ad}_{g} X}, \xi_{\operatorname{Ad}_{g} Y}\right)=\left\langle\operatorname{Ad}_{g}^{*} \ell,\left[\operatorname{Ad}_{g} X, \operatorname{Ad}_{g} Y\right]\right\rangle=\langle\ell,[X, Y]\rangle=\omega_{\ell}\left(\xi_{X}, \xi_{Y}\right),
$$


which shows that $\omega$ is invariant under $G$.

From (2.8) it follows that at the point $n$ giving rise to the orbit $\mathscr{O}_{n}$ one has

$$
\omega_{n}\left(\xi_{X}, \xi_{Y}\right)=\langle n,[X, Y]\rangle \text {. }
$$

The right hand side of this formula is a bilinear skew-symmetric form on $\mathfrak{g}$ which kernel coincides with $\mathfrak{g}_{n}$. This form is non-degenerate on the factor space $\mathfrak{g} / \mathfrak{g}_{n}$ that is isomorphic to the tangent space to the orbit at $n$. Since the action of $G$ on $\mathscr{O}_{n}$ is transitive and preserves $\omega$, this form is non-degenerate at any point of $\mathscr{O}_{n}$ and, therefore, any coadjoint orbit is a symplectic leaf of the Kirillov-Kostant bracket. As a consequence, any coadjoint orbit is even-dimensional. Functions on $\mathfrak{g}^{*}$ invariant under the coadjoint action are obviously constant on any coadjoint orbit and, therefore, they are Casimir functions of the Kirillov-Kostant bracket. ${ }^{12}$

\subsection{Hamiltonian reduction}

Let $\mathscr{P}$ be a symplectic manifold. The hamiltonian or symplectic reduction is a procedure of obtaining a new symplectic manifold $\mathscr{P}_{r}$ from $\mathscr{P}$ by means of reduction over the symplectic action of a Lie group $G$.

Given on $\mathscr{P}$ a dynamical system with a hamiltonian $H$ invariant under a continuous symmetry group, Noether's theorem gives rise to integrals of motion corresponding to this symmetry. Reduction consists in eliminating some degrees of freedom by setting these integrals to constant values. Initial dynamics restricted to the corresponding locus of the phase space is typically degenerate and to obtain a well-defined dynamical system, one has to factor out some additional degrees of freedom. Integrability of the reduced system, if present, is conventionally inherited from some simple and solvable dynamics on the initial phase space. Here we explain the basics of hamiltonian reduction traditionally founded on the geometric notion of the moment map. Further subtle details together with a number of important applications can be found in $[1,8]$.

Hamiltonian action of a Lie group. Let $\mathscr{P}$ be a connected manifold. Suppose $\mathscr{P}$ is endowed with a smooth action of a Lie group $G: G \times \mathscr{P} \rightarrow \mathscr{P}$. Denote by $g \cdot x$ the image of $x \in \mathscr{P}$ under the action of $g$. Then for any $g_{1}, g_{2}$ from $G$ we have $\left(g_{1} g_{2}\right) \cdot x=g_{1} \cdot\left(g_{2} \cdot x\right)$ and $e \cdot x=x$, where $e$ is the identity element. This action induces a representation of $G$ in the space $\mathscr{F}(\mathscr{P})$ :

$$
T(g) f(x)=f\left(g^{-1} \cdot x\right), \quad f \in \mathscr{F}(\mathscr{P}) .
$$

Any element $X$ of the Lie algebra $\mathfrak{g}$ of $G$ gives rise to the vector field $\xi_{X}$ according to

$$
\left(\xi_{X} f\right)(x)=\left.\frac{d}{d t} f\left(e^{-t X} \cdot x\right)\right|_{t=0} .
$$

Since $T(g)$ is a representation, the map $X \rightarrow \xi_{X}$ is a Lie algebra homomorphism $\mathfrak{g} \rightarrow \mathfrak{X}(\mathscr{P})$ meaning that

$$
\left[\xi_{X}, \xi_{Y}\right]=\xi_{[X, Y]} .
$$

\footnotetext{
${ }^{12}$ Any function $f \in \mathscr{F}(\mathfrak{g})$ invariant under coadjoint action has the gradient $\nabla f$ for which $\langle\ell,[X, \nabla f]\rangle=0$ for any $X \in \mathfrak{g}$, as follows from (2.5).
} 
An example of the group action is provided by the coadjoint representation of $G$ in $\mathscr{P}=\mathfrak{g}^{*}$. For this example formula (2.11) turns into (2.5).

Let $\mathscr{P}$ be a symplectic manifold with the 2 -form $\omega$. The action of $G$ on $\mathscr{P}$ is called hamiltonian, if for any $X \in \mathfrak{g}$ the corresponding vector field $\xi_{X}$ is hamiltonian, i.e. there exists a single-valued function $f_{X} \in \mathscr{F}(\mathscr{P})$ such that

$$
i_{\xi_{X}} \omega+d f_{X}=0 .
$$

Since $\xi_{X}$ is hamiltonian, it generates a symplectic transformation. In other words, a hamiltonian action of $G$ on $\mathscr{P}$ is symplectic. Vice versa, at least locally, symplectic transformations by $G$ are hamiltonian [3].

The hamiltonian function $f_{X}$ is determined by (2.13) up to an arbitrary constant which can always be chosen such that the dependence of $f_{X}$ on $X$ is linear. ${ }^{13}$ This choice is not however unique as one can shift $f_{X} \rightarrow f_{X}+\langle\ell, X\rangle$ for any $x$-independent $\ell \in \mathfrak{g}^{*}$ without violating neither (2.13) nor linearity in $X$. Fixing then a concrete linear map $X \rightarrow f_{X}$, we will find that it satisfies the following relation

$$
f_{[X, Y]}=\left\{f_{X}, f_{Y}\right\}+c(X, Y) .
$$

Here $c$ is a bilinear skew-symmetric 2 -form on $\mathfrak{g}$ that is a constant on $\mathscr{P}$, i.e. its value does not depend on a point $x \in \mathscr{P}$. To prove (2.14), we write (2.13) for a Lie algebra element $[X, Y]$

$$
i_{\xi_{[X, Y]}} \omega+d f_{[X, Y]}=0
$$

Since $\xi_{[X, Y]}=\left[\xi_{X}, \xi_{Y}\right]$, we have $i_{\left[\xi_{X}, \xi_{Y}\right]} \omega+d f_{[X, Y]}=0$. But a general property of hamiltonian fields is that $\left[\xi_{f}, \xi_{g}\right]=\xi_{\{f, g\}}$ and since $\xi_{X} \equiv \xi_{f_{X}}{ }^{14}$, we will have that $\left[\xi_{X}, \xi_{Y}\right]=\xi_{\left\{f_{X}, f_{Y}\right\}}$ and, therefore,

$$
i_{\xi_{\left\{f_{X}, f_{Y}\right\}}} \omega+d f_{[X, Y]}=0
$$

On the other hand, by the definition of the hamiltonian vector field

$$
i_{\xi_{\left\{f_{X}, f_{Y}\right\}}} \omega+d\left\{f_{X}, f_{Y}\right\}=0
$$

Comparing (2.15) with (2.16), we conclude that $d\left\{f_{X}, f_{Y}\right\}=d f_{[X, Y]}$, implying (2.14). The Jacobi identity for the Poisson bracket yields for $c$ an equation

$$
c([X, Y], Z)+c([Y, Z], X)+c([Z, X], Y)=0
$$

meaning that $c$ is a 2-cocycle: $\mathfrak{g} \wedge \mathfrak{g} \rightarrow \mathbb{R}$ defining an element of the second cohomology class $H^{2}(\mathfrak{g}, \mathbb{R})$. A trivial cocycle (coboundary) corresponds to $c(X, Y)=\langle\ell,[X, Y]\rangle$ for some $\ell \in \mathfrak{g}^{*}$ and it can always be eliminated from (2.14) by redefining the hamiltonian function as $f_{X} \rightarrow f_{X}-\langle\ell, X\rangle$.

\footnotetext{
${ }^{13}$ Linearity of the map $X \rightarrow f_{X}$ is achieved by first picking particular hamiltonian functions for all elements in a basis of $\mathfrak{g}$ and extending to all $X \in \mathfrak{g}$ by linearity.

${ }^{14}$ This identification is applied precisely after the linear map $X \rightarrow f_{X}$ gets fixed.
} 
Moment map. For any $x \in \mathscr{P}$ a correspondence $X \rightarrow f_{X}(x)$ defines a linear functional on $\mathfrak{g}$ which can be identified with some $\mu \in \mathfrak{g}^{*}$. Explicitly,

$$
\langle\mu(x), X\rangle=f_{X}(x) .
$$

This relation gives rise to a map $\mu$ from the symplectic manifold into the dual space to the Lie algebra

$$
\mu: \mathscr{P} \rightarrow \mathfrak{g}^{*},
$$

known as the moment map.

We further assume that the hamiltonian action of $G$ on a connected manifold $\mathscr{P}$ is such that the hamiltonian functions can be chosen to satisfy

$$
\left\{f_{X}, f_{Y}\right\}=f_{[X, Y]} \cdot
$$

In particular, this is always the case when the second cohomology class is trivial. Formula (2.19) means that the linear map $X \rightarrow f_{X}$ is a homomorphism of the Lie algebra $\mathfrak{g}$ into the Lie algebra of hamiltonian functions. For the freedom $f_{X} \rightarrow f_{X}+\langle\ell, X\rangle$ to be compatible with (2.19), the map $\ell: \mathfrak{g} \rightarrow \mathbb{R}$ must be a 1-cocycle, i.e. it must satisfy the condition $\langle\ell,[X, Y]\rangle=0$ for any $X, Y \in \mathfrak{g}$. Consequently, the space of such $\ell$ 's coincides the first cohomology class $H^{1}(\mathfrak{g}, \mathbb{R}) \simeq(\mathfrak{g} /[\mathfrak{g}, \mathfrak{g}])^{*}$. For semi-simple Lie algebras $[\mathfrak{g}, \mathfrak{g}]=\mathfrak{g}$ and therefore $H^{1}(\mathfrak{g}, \mathbb{R})=0$, which implies that the map $X \rightarrow f_{X}$ obeying (2.19) is uniquely defined. From (2.17) it is then follows that the moment map (2.18) is also uniquely defined for this case.

According to its definition, the quantity $\langle\mu, X\rangle$ is a Poisson algebra generator of the hamiltonian group action

$$
\{\langle\mu, X\rangle, \cdot\}=\xi_{X}
$$

From linearity of the Poisson bracket, for any function $f$ on the phase space one has

$$
\langle\{\mu, f\}, X\rangle=\xi_{X} f \quad \forall X \in \mathfrak{g} .
$$

Evidently, functions invariant under the group action Poisson-commute with the $\mathfrak{g}^{*}$-valued function $\mu(x)$. Below we point out the two most important and interrelated properties of the moment map.

First, condition (2.19) implies that the moment map $\mu: \mathscr{P} \rightarrow \mathfrak{g}^{*}$ is a Poisson map provided the algebra of functions on $\mathfrak{g}^{*}$ is equipped with the Kirillov-Kostant bracket. The left hand side of (2.19) can be written in the form

$$
\left\{f_{X}, f_{Y}\right\}(x)=\langle X \otimes Y,\{\mu \stackrel{\otimes}{,} \mu\}(x)\rangle .
$$

Here the symbol of tensor product within the Poisson bracket indicates that its arguments are regarded as elements of two different vector spaces. For the right hand side of (2.19) one gets

$$
f_{[X, Y]}(x)=\langle[X, Y], \mu(x)\rangle=\left\langle X \otimes Y,\left\langle\mu(x),\left[e_{i}, e_{j}\right]\right\rangle e^{i} \wedge e^{j}\right\rangle .
$$

By comparing we conclude that

$$
\{\mu \stackrel{\otimes}{,} \mu\}(x)=\left\langle\mu(x),\left[e_{i}, e_{j}\right]\right\rangle e^{i} \wedge e^{j},
$$


where the bracket in the left hand side is evaluated on $\mathscr{P}$. This shows that if we endow $\mathfrak{g}^{*}$ with the following Poisson bracket

$$
\{\mu \stackrel{\otimes}{, \mu}\}_{\mathfrak{g}^{*}}=\left\langle\mu,\left[e_{i}, e_{j}\right]\right\rangle e^{i} \wedge e^{j},
$$

then (2.18) is a Poisson map. Obviously, (2.24) is the Kirillov-Kostant bracket (2.3) for the coordinates $\mu_{i}$ on $\mathfrak{g}^{*}$ amalgamated into

$$
\mu=\mu_{i} e^{i}
$$

Second, (2.19) implies that the moment map is $G$-equivariant. This has the following meaning. Let $g(t)$ be a one-parametric subgroup corresponding to $X \in \mathfrak{g}$. According to the definition (2.11), a shift $x \rightarrow g(t)^{-1} \cdot x$ is generated by the vector field $\xi_{X}$. This vector field acts on the moment map as follows

$$
\left\langle\xi_{X} \mu(x), Y\right\rangle=\xi_{X} f_{Y}(x)=\left\{f_{X}, f_{Y}\right\}(x)=f_{[X, Y]}(x)=\langle\mu(x),[X, Y]\rangle,
$$

from which we deduce that

$$
\xi_{X} \mu(x)=-\operatorname{ad}_{X}^{*} \mu(x) .
$$

The global version of this action is $\mu\left(g^{-1} \cdot x\right)=\operatorname{Ad}_{g^{-1}}^{*} \mu(x)$ or, upon replacing $g \rightarrow g^{-1}$,

$$
\mu(g \cdot x)=\operatorname{Ad}_{g}^{*} \mu(x)
$$

Thus, the moment map intertwines the group action on $\mathscr{P}$ with the coadjoint action so that an orbit of $G$ in $\mathscr{P}$ is mapped under $\mu$ into a coadjoint orbit in $\mathfrak{g}^{*}$.

To summarise, the moment map (2.18) is a $G$-equivariant mapping of Poisson manifolds.

Marsden-Weinstein theorem. Let $\mathscr{P}$ be a symplectic manifold with the 2 -form $\omega$. For a fixed $m \in \mathfrak{g}^{*}$ denote by $G_{m} \subset G$ its stabiliser under the coadjoint action. Consider the inverse image $\mu^{-1}(m) \subset \mathscr{P}$. This subspace is invariant under the action of $G_{m}$. Indeed, for $g \in G_{m}$ and $x \in \mu^{-1}(m)$ one has

$$
\mu(g \cdot x)=\operatorname{Ad}_{g}^{*} \mu(x)=\operatorname{Ad}_{g}^{*} m=m,
$$

that is $g \cdot x \in \mu^{-1}(m)$. Thus, one can define the quotient

$$
\mathscr{P}_{r}=\mu^{-1}(m) / G_{m}
$$

This quotient is usually referred to as the reduced phase space, see Fig. 2. If $m$ is chosen such that the action of $G_{m}$ on $\mu^{-1}(m)$ is free and proper, then according to the known theorem $\mathscr{P}_{r}$ is a smooth manifold. The Marsden-Weinstein theorem [8] is a statement that $\mathscr{P}_{r}$ is a symplectic manifold with the symplectic structure inherited from $\omega$ on $\mathscr{P}$.

To get an idea of the proof of the Marsden-Weinstein theorem, let us evaluate $\omega$ on the vector field $\xi_{i} \equiv \xi_{e_{i}}$ of a basis element $e_{i}$ and an arbitrary vector field $\eta$. We have

$$
\omega\left(\xi_{i}, \eta\right)=-d \mu_{i}(\eta)=-\eta \mu_{i}
$$




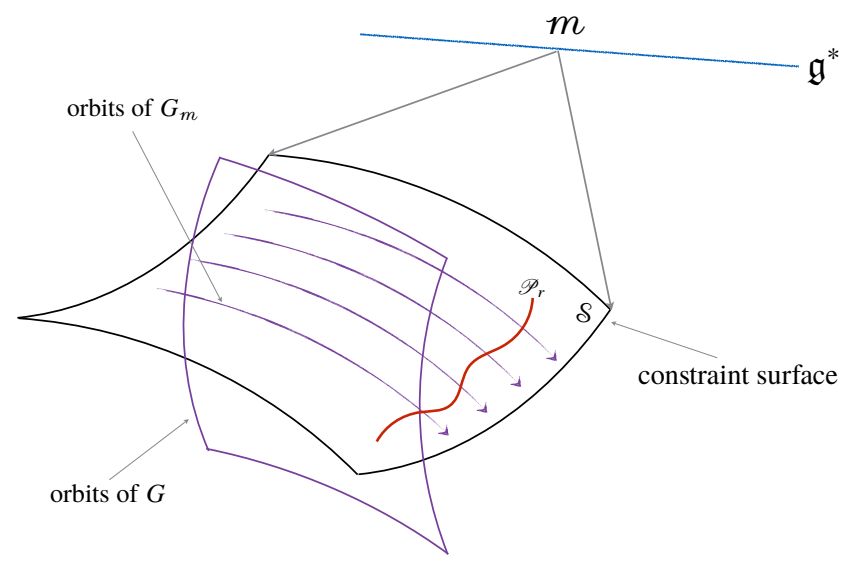

Figure 1: Geometric picture of reduction.

because $\xi_{i}$ has $\mu_{i}(x)$ as its hamiltonian function, see (2.25). If we further assume that $\eta$ is tangent to $\mu^{-1}(m)$, then

$$
\omega_{x}\left(\xi_{i}, \eta\right)=-\left.\eta \mu_{i}\right|_{\mu(x)=m}=0 .
$$

Since $\xi_{i}$ span at $x \in \mu^{-1}(m)$ the tangent space to the orbit of $G$ through $x$, this space is a skew-orthogonal complement of the tangent space to the level set $\delta \equiv \mu^{-1}(m)$

$$
T_{x} \mathcal{S}=T_{x}(G \cdot x)^{\perp} .
$$

Since $\omega$ is non-degenerate,

$$
T_{x} \mathcal{S}^{\perp}=\left(T_{x}(G \cdot x)^{\perp}\right)^{\perp}=T_{x}(G \cdot x),
$$

i.e. these two tangent spaces are orthogonal complements to each other. Obviously,

$$
T_{x} \delta \cap T_{x}(G \cdot x)=T_{x}\left(G_{m} \cdot x\right) .
$$

Thus, the kernel of $\omega$ restricted on $\delta$ is spanned at $x \in \mathcal{S}$ by all the vectors tangent to the orbit of $G_{m}$ at this point. ${ }^{15}$ This allows one to define a non-degenerate closed 2-form $\Omega$ as

$$
\Omega_{x}([\xi],[\eta])=\omega_{x}(\xi, \eta),
$$

where $\xi, \eta$ are vectors tangent to $\mu^{-1}(m)$ at $x$, and $[\xi],[\eta]$ are their equivalence classes defined modulo vectors from $T\left(G_{m} \cdot x\right)$. This form does not depend on a point $x$ along $G_{m}$, because $\omega$ is invariant under $G_{m}: \omega_{g \cdot x}\left(g_{*} \xi, g_{*} \eta\right)=\omega_{x}(\xi, \eta)$ for $g \in G_{m}$. Hence $\Omega$ is a well-defined form on $\mathscr{P}_{r}$ which equips it with the structure of a symplectic manifold. If $\mu$ is a submersion, then the dimension of $\mathscr{P}_{r}$ is

$$
\operatorname{dim} \mathscr{P}_{r}=\operatorname{dim} \mathscr{P}-\operatorname{dim} \mathfrak{g}-\operatorname{dim} \mathfrak{g}_{m},
$$

which is ipso facto an even number.

\footnotetext{
${ }^{15}$ In particular, any orbit of $G_{m}$ in $\delta$ is an isotropic submanifold, as the symplectic form evaluated on any two vector fields $\xi, \eta$ tangent to an orbit vanishes: $\omega_{x}(\xi, \eta)=0, x \in \mathcal{S}$.
} 
The symplectic form $\Omega$ can be effectively inverted with the help of the Dirac bracket construction. According to (2.23), the Poisson brackets of $\mu_{i}(x)$ 's reduced on the constraint surface take the form

$$
\left.\left\{\mu_{i}, \mu_{j}\right\}(x)\right|_{x \in \mathcal{S}}=\left.\left\langle\mu(x),\left[e_{i}, e_{j}\right]\right\rangle\right|_{\mu(x)=m}=\left\langle\operatorname{ad}_{e_{j}}^{*} m, e_{i}\right\rangle .
$$

We arrange basis vectors $\left\{e_{i}\right\}$ of $\mathfrak{g}$ such that the first $\operatorname{dim} \mathfrak{g}_{m}$ vectors with $i=1, \ldots, \operatorname{dim} \mathfrak{g}_{m}$ constitute a basis of $\mathfrak{g}_{m}$, where $\mathfrak{g}_{m} \subset \mathfrak{g}$ is the Lie algebra of the stabiliser $G_{m}$ of $m$. In the case $e_{j} \in \mathfrak{g}_{m}$ the bracket (2.30) vanishes for any $e_{i} \in \mathfrak{g}$. This observation motivates to split all the constraints into two sets

$$
\left\{\mu_{i}(x)\right\}=\left\{\mu_{\alpha}(x), \alpha \in 1, \ldots, \operatorname{dim} \mathfrak{g}_{m}\right\} \cup\left\{\mu_{\bar{\alpha}}(x), \bar{\alpha} \in 1, \ldots, \operatorname{dim}\left(\mathfrak{g} / \mathfrak{g}_{m}\right)\right\} .
$$

Constraints from the first set are called first class, they have the characteristic property that their Poisson bracket with any constraint vanishes on the constraint surface. Transformations of the phase space induced by the hamiltonian vector fields of $\mu_{\alpha}$ are known in physics context as gauge transformations. These transformations form a gauge group which, by construction, coincides with the stabiliser $G_{m}$ of the fixed value $m$ of the moment map.

The constraints from the second set are called second class, the matrix $\Psi_{\bar{\alpha} \bar{\beta}}=\left\{\mu_{\bar{\alpha}}, \mu_{\bar{\beta}}\right\}$ is invertible in some neighbourhood of the constraint surface. The hamiltonian vector fields $\xi_{\bar{\alpha}}$ corresponding to functions $\mu_{\bar{\alpha}}$ are "transversal" to $\delta$. For any two functions $f$ and $h$ on $\mathscr{P}$ the Dirac bracket is then defined as

$$
\{f, h\}_{\mathrm{D}}=\{f, h\}-\left\{f, \mu_{\bar{\alpha}}\right\} \Psi_{\bar{\alpha} \bar{\beta}}^{-1}\left\{\mu_{\bar{\beta}}, h\right\} .
$$

One can show that this bracket obeys the Jacobi identity and its inverse on $\mathscr{P}_{r}$ coincides with $\Omega$.

Invariant dynamics. The Marsden-Weinstein theorem gives a construction of a symplectic manifold from another one equipped with a hamiltonian action of a Lie group $G$. Let on a symplectic manifold $\mathscr{P}$ a dynamical system be given with a hamiltonian $H$ invariant under the action of $G$. Let $\xi_{X}$ be the hamiltonian vector field of $X \in \mathfrak{g}$. The invariance of $H$ means that

$$
-\xi_{X} H=\left\{H, f_{X}\right\}=\frac{d f_{X}}{d t}=0,
$$

where $t$ is the time parameter along the flow generated by $H$. Hence, the hamiltonian functions $f_{X}$ are integrals of motion and, as a consequence, the moment map (2.17) remains constant along the trajectories of the dynamical system $\mu(x(t))=$ const. Equation (2.33) is a hamiltonian analogue of Noether's theorem that provides a relation between variational symmetries and conservation laws. From a geometric point of view, conservation of the momentum map means that if the starting point of a trajectory of the hamiltonian system is on the surface $\mu(x)=m$, then the whole trajectory lies on this surface.

Coadjoint orbits from reduction of $\mathrm{T}^{*} G$. As a straightforward application of the hamiltonian reduction technique, we consider the reduction of $\mathrm{T}^{*} G$ with respect to the left or right action of $G$, which in both cases produces coadjoint orbits as reduced phase spaces. Recall that the left and right actions of $G$ on $\mathrm{T}^{*} G \simeq G \times \mathfrak{g}^{*}$ are

$$
h \cdot(g, \ell)=(h g, \ell), \quad(g, \ell) \cdot h^{-1}=\left(g h^{-1}, \operatorname{Ad}_{h}^{*} \ell\right),
$$


where $h \in G$ and used employed the left parameterisation of the cotangent bundle by a pair $(g, \ell)$. By using (1.42) one can verify that the moment maps of these actions are

$$
\mu_{l}(g, \ell)=-\operatorname{Ad}_{g}^{*} \ell=-m, \quad \mu_{r}(g, \ell)=\ell,
$$

see, for instance, [9] for details. For the reduction under the left action, we fix the moment map $\mu_{\iota}$ to a constant value $-m_{0}$. The constraint surface $\delta=\mu_{l}^{-1}\left(m_{0}\right)$ is then

$$
\mathcal{S}=\left\{\left(g, \mathrm{Ad}_{g^{-1}}^{*} m_{0}\right), \quad g \in G\right\} \simeq G .
$$

If $G_{m_{0}}$ is an isotropy subgroup of $m_{0}$, then the reduced phase space can be either modelled as a left coset $\mathscr{P}_{r}=G_{m_{0}} \backslash G$ or alternatively as the coadjoint orbit $\mathscr{P}_{r}=\left\{\operatorname{Ad}_{g^{-1}}^{*} m_{0}, g \in G\right\}=\mathscr{O}_{m_{0}}$ through $m_{0}$. Note that this kind of reduction is physically realised in the case of hamiltonian dynamics of Euler's top. Fixing $m_{0}$ is the same as fixing the value of the conserved angular momentum $J$ in the stationary frame. Identifying $\mathfrak{g}^{*}$ with $\mathfrak{g}$ by means of the non-degenerate bilinear form $(a, b)=\operatorname{Tr}(a b)$, where $a, b \in \operatorname{so}(n)$, we obtain a coadjoint orbit $\ell=g^{-1} J g$ which is the phase space for Euler's top.

For the right action the moment map $\mu_{r}$ coincides with the projection of an element $(g, \ell)$ on its second component and fixing it to a constant value $\ell_{0}$ gives $\delta \simeq G \times \ell_{0} \simeq G$. The reduced phase space is a coadjoint orbit $\mathscr{O}_{\ell_{0}}$ which is modelled this time by the right coset $\mathscr{P}_{r}=G / G_{\ell_{0}}$.

\subsection{Hyperbolic CMS model from reduction}

Here we consider an interesting application of the reduction technique for deriving an integrable of the CMS type from $\mathrm{T}^{*} G$. Historically, this derivation goes back to [10], where the corresponding models with rational and trigonometric potentials have been obtained from $\mathrm{T}^{*} \mathfrak{g}$ and $\mathrm{T}^{*} G$, respectively. Since we are mostly interested in the algebraic aspects, we neglect the issue of reality conditions by working within algebraic (holomorphic) approach, where the role of the phase space is played by a complex algebraic manifold [11]. Adopting this approach and staring from $\mathrm{T}^{*} G$ as an initial phase space, we derive the CMS model of type III.

We take $G=\operatorname{GL}(N, \mathbb{C})$ and consider the combined left-right action of $G$ on $\mathrm{T}^{*} G$, namely,

$$
h \cdot(g, \ell) \cdot h^{-1}=\left(h g h^{-1}, \operatorname{Ad}_{h}^{*} \ell\right) .
$$

It follows from our considerations that this action is hamiltonian and the corresponding moment map $\mu$ is obtained as the sum of the moment maps for the left and right actions

$$
\mu=\ell-g \ell g^{-1},
$$

where we have identified $\mathfrak{g}^{*}$ with $\mathfrak{g}=\operatorname{Mat}(N, \mathbb{C})$ so that the coadjoint action becomes equivalent to the adjoint one. We choose a value $n$ of the moment map such that it would have the stabiliser $G_{n}$ of maximal dimension, so that the corresponding coadjoint orbit would have the minimal dimension. We therefore take

$$
n=\boldsymbol{e} \otimes \boldsymbol{e}^{t}-\mathbb{1} .
$$

where $\boldsymbol{e}$ is a $N$-dimension vector with all entries equal to one. Indeed, $n$ has $N-1$ coincident eigenvalues, $G_{n} \simeq \mathrm{GL}(1) \times \mathrm{GL}(N-1)$ and, therefore, the orbit in $\mathfrak{g}^{*}$ we are interested in, is 
isomorphic to the coset $\mathscr{O}_{n} \simeq \mathrm{GL}(N) /(\mathrm{GL}(1) \times \mathrm{GL}(N-1))$. This orbit has a complex dimension $2(N-1)$ and is among the orbits of minimal positive dimension. Thus, the moment map equation we will consider is

$$
\ell-g \ell g^{-1}=i \gamma\left(\boldsymbol{e} \otimes \boldsymbol{e}^{t}-\mathbb{1}\right),
$$

where $\gamma$ is an arbitrary constant. To solve this equation, we restrict ourselves to diagonalisable elements $g \in G$ which admit the following representation

$$
g=T Q T^{-1},
$$

where $Q$ is a diagonal matrix with pairwise different non-vanishing eigenvalues. To fix matrix $T \in G$ uniquely, we requite it to obey the so-called Frobenius condition [12]

$$
T e=e .
$$

Obviously, the set of matrices satisfying this condition form a group $F$. The Lie algebra $\mathfrak{f}$ of this group is Frobenius, i.e. it admits a a non-degenerate 2-cocycle that is coboundary, which explains the name "Frobenius condition" for (2.37). The group $F$ is then called Frobenius group.

Substituting (2.36) into (2.35) and multiplying (2.35) with $T^{-1}$ from the left and with $T$ from the right, we get

$$
L-Q L Q^{-1}=i \gamma\left(\boldsymbol{e} \otimes \boldsymbol{e}^{t} T-\mathbb{1}\right),
$$

where we have introduced

$$
L=T^{-1} \ell T \in \mathfrak{g} .
$$

First, we note that the diagonal part of the left hand side of (2.38) vanishes which for the right hand side implies the condition $\boldsymbol{e}^{t} T=0$. With this condition at hand, we project (2.38) on the off-diagonal part and solve the corresponding equation for $L_{i j}$

$$
L_{i j}=-i \gamma \frac{Q_{j}}{Q_{i j}}, \quad i \neq j,
$$

with $Q_{i j}=Q_{i}-Q_{j}$. The diagonal part of $L$ decouples from (2.38) and therefore remains arbitrary. Hence, for $L$ we find the following solution

$$
L=\sum_{i=1}^{N} p_{i} E_{i i}-i \gamma \sum_{i \neq j}^{N} \frac{Q_{j}}{Q_{i j}} E_{i j},
$$

which is obviously the same that matrix $L$ from the Lax pair (1.57) for the hyperbolic CMS model. The stabiliser $G_{n}$ consists of the one dimensional center of GL( $\left.N\right)$ together with a group defined by two conditions

$$
\left\{T \in G: T e=e, e^{t} T=e^{t}\right\} \simeq \operatorname{GL}(N-1) .
$$

Because of the center, the coadjoint (adjoint) action of GL(N) is not effective and as the result equation (2.35) comprises $N^{2}-1$ constraints rather than $N$, as is seen from the fact that the trace of 
(2.35) is automatically zero and does not lead to a separate constraint. For this reason the dimension of the reduced phase space is computed by the formula which is slightly different from (2.29) and takes into account the presence of the center

$\operatorname{dim} \mathscr{P}_{r}=\operatorname{dim}\left(T^{*} G\right)-\operatorname{dim}(\mathrm{GL}(N) / \mathrm{GL}(1))-\operatorname{dim}(\mathrm{G}(N-1))=2 N^{2}-\left(N^{2}-1\right)-(N-1)^{2}=2 N$.

These $2 N$ degrees of freedom are precisely $n$ variables $Q_{i}$ and $n$ variables $p_{i}$, and they parametrise the reduced phase space. Moreover, we can now see the geometric origin of the Lax matrix: its entries are functions on the cotangent bundle invariant under the action of $G_{n}$. Indeed, under $h \in G_{n}$, we have

$$
\ell \rightarrow h \ell h^{-1}, \quad T \rightarrow h T,
$$

in particular, here the transformation for $T$ follows form the fact that a non-central $h \in G_{n}$ is an element of the Frobenius group. Under (2.43) the quantity $L=T^{-1} \ell T$ remains invariant.

To complete the discussion of the reduced phase space, we need to establish the Poisson structure between the variables $p_{i}, Q_{i}$. Starting from (1.42), computation of the Poisson brackets of $T, Q$ and $\ell$ yields

$$
\begin{array}{llrl}
\left\{T_{1}, \ell_{2}\right\} & =T_{1} T_{2} s_{12} T_{2}^{-1}, & & \left\{Q_{1}, \ell_{2}\right\}=-Q_{1} T_{2} \bar{C}_{12} T_{2}^{-1}, \\
\left\{Q_{1}, T_{2}\right\}=0, & & \left\{Q_{1}, Q_{2}\right\}=0 .
\end{array}
$$

where

$$
s_{12}=-\sum_{i \neq j}^{N} \frac{Q_{i}}{Q_{i j}}\left(E_{i i}-E_{i j}\right) \otimes E_{j i}, \quad \bar{C}_{12}=\sum_{i=1}^{N} E_{i i} \otimes E_{i i} .
$$

In deriving these formulae the Frobenius condition was essentially used, see [9] for details. Now we obtain

$$
\left\{Q_{1}, L_{2}\right\}=\left\{Q_{1}, T_{2}^{-1} \ell_{2} T_{2}\right\}=-Q_{1} \bar{C}_{12}
$$

From the structure of the right hand, it is clear that only the diagonal part of $L$ contributes which allows one to conclude that on the reduced phase space one has $\left\{Q_{i}, p_{j}\right\}=-Q_{i} \delta_{i j}$. If we parametrise $Q_{i}=e^{q_{i}}$, then the last formula implies the canonical bracket $\left\{p_{i}, q_{j}\right\}=\delta_{i j}$.

We can further compute the Poisson bracket between components of the Lax matrix. We find

$$
\left\{L_{1}, L_{2}\right\}=\left[r_{12}, L_{1}\right]-\left[r_{21}, L_{2}\right]
$$

where $r_{12}=\frac{1}{2} C_{12}-s_{12}$, which explicitly reads as

$$
r_{12}=\frac{1}{2} \sum_{i, j}^{N} E_{i j} \otimes E_{j i}+\sum_{i \neq j}^{N} \frac{Q_{i}}{Q_{i j}}\left(E_{i i}-E_{i j}\right) \otimes E_{j i} .
$$

Thus, the Poisson brackets between entries of the Lax matrix admit the required $r$-matrix form to guarantee involutivity of $I H_{k}=\operatorname{Tr} L^{k}$. Clearly, the $r$-matrix is dynamical as it depends on $Q_{i}$. 
Concerning the dynamics, we can take any invariant function of $\ell$ as the hamiltonian $H$. Since invariants of $\ell$ are Casimirs of the Kirillov-Kostant bracket for $\ell$, the evolution equation for $L=T^{-1} \ell T$ with any such hamiltonian will take the form of the Lax equation

$$
\frac{d L}{d t}=\{H, L\}=[M, L], \quad M=-T^{-1} \dot{T}
$$

as $\dot{\ell}=0$. Here the element $T \equiv T(t)$ takes values in the space of solutions (2.42) of the moment map equation (2.35). As a result, $M$ can always be chosen to satisfy two conditions

$$
M e=0, \quad \boldsymbol{e}^{t} M=0
$$

As an example, consider the hamiltonian $H=\frac{1}{2} \operatorname{Tr} \ell^{2}=\frac{1}{2} \operatorname{Tr} L^{2}$. The Poisson bracket of $H$ with $L$ is easily computed from (2.44) and one finds

$$
M=\operatorname{Tr}_{1}\left(L_{1} s_{21}\right)=i \gamma \sum_{i \neq j}^{N} \frac{Q_{i} Q_{j}}{Q_{i j}^{2}}\left(E_{i i}-E_{i j}\right) .
$$

Evidently, this matrix satisfies the conditions (2.50). As a consequence, $M$ commutes with the element $\boldsymbol{e} \otimes \boldsymbol{e}^{t}=\sum_{i j} E_{i j}$.

Let us now show that solving equations of motion reduces to a variant of the factorisation problem. On the initial phase space the equations of motion triggered by $H=\frac{1}{2} \operatorname{Tr} \ell^{2}$

$$
\dot{g}=g \ell, \quad \dot{\ell}=0
$$

are trivially solved

$$
\dot{g}=Q(0) e^{t \ell}, \quad \ell=\text { const },
$$

where we assumed that at $t=0$ the point $g$ lies on the reduced phase space. Then at any later moment of time, the exponentiated coordinates $Q(t)$ are determined by solving the factorisation problem

$$
Q(0) e^{t \ell}=T(t) Q(t) T(t)^{-1}, \quad T(t) \boldsymbol{e}=\boldsymbol{e}
$$

with the initial condition $T(0)=\mathbb{1}$. Since $L(t)=T^{-1}(t) \ell T(t)$, we can identify $\ell=L(0)$, so that $\ell$ encodes the information on the initial coordinates and momenta. Once $T(t)$ is found by algebraic factorisation, one can compute $p_{i}(t)$ by projecting $L(t)=T^{-1}(t) L(0) T(t)$ on its diagonal part. In this way solving the differential equations of motion was reduced to algebraic operations, hence to quadrature.

This completes our derivation of the CMS model by means of the reduction from $\mathrm{T}^{*} G$. For recent applications of more involved reduction techniques which use the Poisson action of PoissonLie groups and derivation in this context the relativistic generalisations of the (spin) CMS models, known as the Ruijsenaars-Schneider (RS) models [13], see [14, 15] and the references therein. 


\section{Quantum integrability I: Discrete spectrum}

Determination of the spectrum of the Schrödinger operator is one of the central tasks of quantum mechanics. The particularity of an integrable model is that the hamiltonian defining the corresponding Schrödinger operator is a member of a commuting family of differential operators, so that one can search for a basis of common eigenfunctions for all of them. Among various approaches to the spectral problem the following two are the most advanced - the first is rooted in the theory of multivariable orthogonal polynomials, and the second is based on a special ansatz for the asymptotic wave function. In particular, the second approach, known as Bethe Ansatz, is applicable to a large variety of integrable models that support scattering, and we address it later. Here we concentrate on the method of orthogonal polynomials, suitable for the case of a purely discrete spectrum. The corresponding models include, for instance, the rational CMS model with a harmonic oscillator potential, the trigonometric CMS and RS models.

\subsection{Quantum trigonometric CMS model}

To illustrate the procedure of constructing the spectrum of an integrable model by using the theory of orthogonal polynomials, we employ the quantum trigonometric CMS model as an example. The hamiltonian of this model is the following Schrödinger operator

$$
H=-\frac{\hbar^{2}}{2} \sum_{i=1}^{N} \partial_{i}^{2}-\frac{\gamma(\gamma-\hbar)}{2 \ell^{2}} \sum_{i \neq j}^{N} \frac{Q_{i} Q_{j}}{Q_{i j}^{2}} .
$$

where $\partial_{i}=\frac{\partial}{\partial q_{i}}$. This operator is obtained from the classical hamiltonian (1.50) by replacing the classical momentum $p_{i}$ with its quantum counterpart $p_{i} \rightarrow-i \hbar \partial_{i}$. We also found convenient to write the potential via the exponential variables $Q_{i}=e^{i q_{i} / \ell}$ that are periodic functions of $q_{i}$ with the period $2 \pi \ell$. The coupling constant $g=\gamma^{2}>0$ of the classical theory was replaced with $g=\gamma(\gamma-\hbar)$ so that in this parametrisation $g$ automatically obeys $g>-\frac{\hbar^{2}}{4}$ for any real $\gamma \neq \frac{\hbar}{2}$. This replacement is related to the fact that in the rational degeneration of the model the negative values of the coupling constant $-\frac{\hbar^{2}}{4}<g<0$ are also allowed.

Commutative operator families. The model is quantum integrable and the hamiltonian is a part of the family of $N$ pair-wise commuting operators. These operators can be constructed as quantisation of the classical integrals (1.61). The first few read as

$$
\begin{aligned}
H_{1} & =(-i \hbar) \sum_{i} \partial_{i}, \\
H_{2} & =(-i \hbar)^{2}\left[\sum_{i} \partial_{i}^{2}+\frac{\beta(\beta-1)}{\ell^{2}} \sum_{i \neq j} \frac{Q_{i} Q_{j}}{Q_{i j}^{2}}\right], \\
H_{3} & =(-i \hbar)^{3}\left[\sum_{i} \partial_{i}^{3}+\frac{3 \beta(\beta-1)}{\ell^{2}} \sum_{i \neq j} \frac{Q_{i} Q_{j}}{Q_{i j}^{2}} \partial_{i}\right], \\
H_{4} & =(-i \hbar)^{4}\left[\sum_{i} \partial_{i}^{4}\right. \\
& +\frac{\beta(\beta-1)}{\ell^{2}} \sum_{i \neq j} \frac{Q_{i} Q_{j}}{Q_{i j}^{2}}\left[4 \partial_{i}^{2}+2 \partial_{i} \partial_{j}-i \frac{Q_{i}+Q_{j}}{\ell Q_{i j}}\left(\partial_{i}-\partial_{j}\right)-\frac{Q_{i}^{2}+Q_{j}^{2}+4 Q_{i} Q_{j}}{\ell^{2} Q_{i j}^{2}}\right]+
\end{aligned}
$$




$$
\left.+\frac{\beta^{2}(\beta-1)^{2}}{\ell^{4}} \sum_{i \neq j} \frac{Q_{i}^{2} Q_{j}^{2}}{Q_{i j}^{4}}+\frac{2 \beta^{2}(\beta-1)^{2}}{\ell^{4}} \sum_{i \neq j \neq k} \frac{Q_{i} Q_{j}^{2} Q_{k}}{Q_{i j}^{2} Q_{j k}^{2}}\right],
$$

where we have introduced the dimensionless coupling constant $\beta=\frac{\gamma}{\hbar}$. In particular, $H_{1}=P$ is the operator of total momentum. Note that starting from $H_{4}$ the classical expressions get $\hbar$-corrections to yield the normal ordered quantum integrals where all momenta (derivatives) are on the right from coordinate-dependent expressions. There is another convenient basis of integrals which allows for a compact general formula for $k^{\prime}$ th integral [16]

$$
D_{k}=\hbar^{k} \sum_{j=0}^{[k / 2]} \frac{(-i)^{k-2 j} \beta^{j}(\beta-1)^{j}}{2^{j} j !(k-2 j) ! \ell^{2 j}} \sum_{i_{1}, \ldots, i_{k}}{ }^{\prime} \frac{Q_{i_{1}} Q_{i_{2}}}{Q_{i_{1} i_{2}}^{2}} \ldots \frac{Q_{i_{2 j-1}} Q_{i_{2 j}}}{Q_{i_{2 j-1} i_{2 j}}^{2}} \partial_{i_{2 j+1}} \partial_{i_{2 j+2}} \ldots \partial_{i_{k}},
$$

where $\Sigma^{\prime}$ means that no two summation indices coincide. In the limit of zero coupling integrals $D_{k}$ turn into symmetric functions of derivatives that justifies for this bases the name symmetric. As is seen from (3.3), the ordering of the coordinate and momentum operators within any individual sum is irrelevant. Introducing the quantum Lax operator

$$
L=-i \hbar \sum_{i} E_{i i} \partial_{i}+\gamma \sum_{i \neq j} \frac{Q_{j}}{Q_{i j}} E_{i j}
$$

one can verify that the integrals $D_{k}$ are generated in the expansion of the following determinant over the formal parameter $\zeta$

$$
\operatorname{det}(\zeta \mathbb{1}-L)=\sum_{k=0}^{N} \zeta^{N-k}(-1)^{k} D_{k}, \quad D_{0}=1,
$$

where $L$ is given by (3.4). As the reader can verify, in computing the above determinant by the Laplace expansion no ordering ambiguity arises. Since classically $L(\gamma)^{t}=Q L(-\gamma) Q^{-1}$, where $Q=\sum_{i} Q_{i} E_{i i}$, the determinant (3.4) is a function of $\gamma^{2}$, so that once the determinant is computed, one needs to substitute $\gamma^{2} \rightarrow \hbar^{2} \beta(\beta-1)$ to get formula (3.3) for $D_{k}$. Finally, the relations between the power sum and symmetric bases of integrals are given by the following determinant formulae

$$
H_{k}=\left|\begin{array}{ccccc}
D_{1} & 1 & 0 & \cdots & 0 \\
2 D_{2} & D_{1} & 1 & \cdots & 0 \\
\vdots & \vdots & . & \cdots & \vdots \\
(k-1) D_{k-1} & D_{k-2} & . & \cdots & 1 \\
k D_{k} & D_{k-1} & . & \cdots & D_{1}
\end{array}\right|, \quad D_{k}=\frac{1}{k !}\left|\begin{array}{ccccc}
H_{1} & 1 & 0 & \cdots & 0 \\
H_{2} & H_{1} & 2 & \cdots & 0 \\
\vdots & \vdots & . & \cdots & \vdots \\
H_{k-1} & H_{k-2} & . & \cdots & k-1 \\
H_{k} & H_{k-1} & . & \cdots & H_{1}
\end{array}\right| .
$$

In fact this are the same formulae that relate the power-sum symmetric functions and elementary symmetric functions.

The boost operator. In addition to the hamiltonian and momentum operator, sometimes it is convenient to consider the operator of the Galilean boost that is defined as

$$
B=\prod_{i=1}^{N} Q_{i}^{S}
$$


where the parameter $s \in \mathbb{R}$ plays the role of the velocity of the moving frame. This operator boosts the hamiltonian to give

$$
B^{-1} H B=H+\frac{s}{\ell} P+\frac{N s^{2}}{2 \ell^{2}} .
$$

More generally, one can study the spectrum of this boosted hamiltonian, which is essentially an admixture of $H$ and $P$, but we will restrict ourselves to the spectrum of $H$.

The ground state. The first step towards the spectrum of the model is to find the ground state wave function and the ground state energy. This can be done by noting that the hamiltonian (3.1) can be represented as

$$
H=\frac{1}{2} \sum_{i=1}^{N} A_{i}^{\dagger} A_{i}+E_{0}
$$

where

$$
A_{i}=-i \hbar \partial_{i}-\frac{\gamma}{2 \ell} \sum_{j \neq i}^{N} \frac{Q_{i}+Q_{j}}{Q_{i}-Q_{j}}, \quad A_{i}^{\dagger}=-i \hbar \partial_{i}+\frac{\gamma}{2 \ell} \sum_{j \neq i}^{N} \frac{Q_{i}+Q_{j}}{Q_{i}-Q_{j}}
$$

are two hermitian conjugate operators and

$$
E_{0}=\frac{\gamma^{2}}{\ell^{2}} \frac{N\left(N^{2}-1\right)}{24}
$$

Since $\sum_{i=1}^{N} A_{i}^{\dagger} A_{i}$ is a hermitian semi-positive operator, $E_{0}$ coincides with the ground state energy, while the ground state wave function $\triangle$, is determined by a compatible system of equations

$$
A_{j} \Delta=0, \quad j=1, \ldots, N .
$$

The solution of this system is a Jastrow wave function

$$
\Delta=\prod_{i<j}^{N}\left(Q_{i}-Q_{j}\right)^{\beta} \prod_{i=1}^{N} Q_{i}^{-(N-1) \beta / 2},
$$

which up to a phase coincides with $\prod_{i<j}^{N}\left|Q_{i}-Q_{j}\right|^{\beta}$.

Note that the ground state energy admits the thermodynamic limit where the length $L=2 \pi \ell$ of the box (period) and the particle number $N$ both tend to infinity, such that the particle density $\mathcal{D}=N / L$ remains finite in this limit. From (3.7) for the density $\mathscr{E}_{0}$ of the ground state energy of the we find in the thermodynamics limit

$$
\mathscr{E}_{0}=\lim _{N, L \rightarrow \infty} \frac{E_{0}}{L}=\frac{(\pi \gamma)^{2}}{6} \mathcal{D}^{3}
$$

Excited states. To find the spectrum of excited states, we pass from the hamiltonian to a new operator $\mathscr{H}$ which is obtained by conjugating $H$ with the ground state wave-function and subtracting the ground state energy: $\mathscr{H} \equiv \frac{2 \ell^{2}}{\hbar^{2}}\left(\triangle^{-1} H \triangle-E_{0}\right)$. Explicitly,

$$
\mathscr{H}=\sum_{i=1}^{N}\left(Q_{i} \frac{\partial}{\partial Q_{i}}\right)^{2}+\beta \sum_{i<j}^{N} \frac{Q_{i}+Q_{j}}{Q_{i}-Q_{j}}\left(Q_{i} \frac{\partial}{\partial Q_{i}}-Q_{j} \frac{\partial}{\partial Q_{j}}\right) .
$$


This operator is invariant under the action of the symmetric group $\mathfrak{S}_{N}$ and its spectrum will be spanned by symmetric polynomials of $Q_{i}$. Indeed, consider the following monomial symmetric functions

$$
\boldsymbol{m}_{\lambda}=\sum_{\sigma \in \mathfrak{S}_{N}} Q_{\sigma(1)}^{\lambda_{1}} Q_{\sigma(2)}^{\lambda_{2}} \cdots Q_{\sigma(N)}^{\lambda_{N}}
$$

associated to the Young diagram $\lambda=\left[\lambda_{1}, \lambda_{2}, \lambda_{3}, \ldots\right]$, where the non-negative integers $\lambda_{i}$ (dominant weights) form a weakly decreasing sequence

$$
\lambda_{1} \geq \lambda_{2} \geq \lambda_{3} \geq \ldots
$$

Applying the operator $\mathscr{H}$ to $\boldsymbol{m}_{\lambda}$ yields

$$
\mathscr{H} \boldsymbol{m}_{\lambda}=\varepsilon_{\lambda} \boldsymbol{m}_{\lambda}+\sum_{\mu<\lambda} c_{\lambda \mu} \boldsymbol{m}_{\mu},
$$

where

$$
\varepsilon_{\lambda}=\sum_{j} \lambda_{j}^{2}+\beta \sum_{i<j}\left(\lambda_{i}-\lambda_{j}\right)
$$

Here we have also used the notion of a dominance partial order defined on a set of partitions as

$$
\mu \leq \lambda \text { if and only if } \mu_{1}+\ldots+\mu_{j} \leq \lambda_{1}+\ldots+\lambda_{j} \text {, for all } j=1, \ldots, N \text {. }
$$

This order is only partial because not all the partitions can be compared in this way.

Thus, $\mathscr{H}$ is triangular in a symmetric monomial basis and its eigenfunctions can be searched as linear combinations of $\boldsymbol{m}_{\lambda}$ with all subordinate $\boldsymbol{m}_{\mu}$. This leads to the construction of the spectrum in terms of symmetric Jack polynomials.

\subsection{Spectrum via Jack polynomials}

For a given partition $\lambda$ denote by $m_{1}$ multiplicity of its part 1 , by $m_{2}$ multiplicity of its part 2 , etc. Let us associate to $\lambda$ the following number

$$
z_{\lambda}=1^{m_{1}} m_{1} ! 2^{m_{2}} m_{2} ! \ldots
$$

With this notation we define on the space of symmetric functions the following scalar product

$$
\left\langle p_{\lambda}, p_{\mu}\right\rangle_{\beta}=\delta_{\lambda, \mu} z_{\lambda} \beta^{-\ell(\lambda)},
$$

where $\ell(\lambda)$ is the length of the partition $\lambda$ (the number of parts). The Jack polynomials $J_{\lambda}(Q ; \beta)$ are known to be uniquely defined by the following conditions [17]

1) $J_{\lambda}(Q ; \beta)=\boldsymbol{m}_{\lambda}+\sum_{\mu<\lambda} u_{\mu \lambda}(\beta) \boldsymbol{m}_{\mu}$,

2) $\left\langle J_{\lambda}, J_{\mu}\right\rangle_{\beta}=0$ if $\lambda \neq \mu$, 
where the coefficients $u_{\mu \lambda}(\beta)$ are rational functions of $\beta$. If $l(\lambda)>N$, then $J_{\lambda}(Q, \beta)=0$. For $\beta=1$ the Jack polynomials coincide with Schur polynomials. The Jack polynomials are orthogonal with respect to the scalar product

$$
\left(f_{1}, f_{2}\right)=\frac{1}{(2 \pi i)^{N}} \prod_{j=1}^{N} \oint_{\left|Q_{j}\right|=1} \frac{\mathrm{d} Q_{j}}{Q_{j}} f_{1}(Q) \overline{f_{2}(Q)} \prod_{i<j}^{N}\left|Q_{i}-Q_{j}\right|^{2 \beta} .
$$

In fact, the scalar product (3.16) is proportional to (3.15). Among other properties of the Jack polynomials the following relation is noteworthy

$$
\left(\prod_{i} Q_{i}\right) J_{\lambda}(Q ; \beta)=J_{\lambda+1}(Q ; \beta), \quad \lambda+1 \equiv\left[\lambda_{1}+1, \lambda_{2}+1, \ldots\right] .
$$

Finally, the Jack polynomials are the eigenstates of $\mathscr{P}$ and $\mathscr{H}$

$$
\mathscr{H} J_{\lambda}(Q ; \beta)=\varepsilon_{\lambda} J_{\lambda}(Q ; \beta), \quad \mathscr{P} J_{\lambda}(Q ; \beta)=p_{\lambda} J_{\lambda}(Q ; \beta),
$$

where eigenvalues $\varepsilon_{\lambda}$ are given by (3.13) and

$$
p_{\lambda}=\sum_{j=1}^{N} \lambda_{j}
$$

Here $\mathscr{P}=\frac{\ell}{\hbar} \triangle^{-1} P \triangle=\frac{\ell}{\hbar} P$ is the operator of total momentum because $P$ annihilates the ground state wave function.

A simple and explicit formula for Jack polynomials is currently unknown. The reader can consult the Table 3.1 in [9] for the list the first seven polynomials together with the corresponding eigenvalues of the operator $\mathscr{H}$. Combing all the pieces together, we obtain the following formula for eigenfunctions of $H$

$$
\Psi_{\lambda}(Q)=\left(\prod_{i=1}^{N} Q_{i}\right)^{-(N-1) \beta / 2} \prod_{i<j}^{N}\left(Q_{i}-Q_{j}\right)^{\beta} J_{\lambda}(Q ; \beta) .
$$

Quasi-momentum. The spectrum of the trigonometric model has a striking interpretation in terms of quasi-particles which makes the integrable structure of the model manifect. Taking into account the expression for the ground state energy, we obtain the following formula for the spectrum of the hamiltonian (3.1)

$$
E=\frac{\hbar^{2}}{2 \ell^{2}} \sum_{j=1}^{N}\left(\lambda_{j}+\beta(N+1-2 j)\right) \lambda_{j}+\frac{\hbar^{2} \beta^{2}}{\ell^{2}} \frac{N\left(N^{2}-1\right)}{24} .
$$

Introducing the dimensionful quasi-momenta ${ }^{16}$

$$
p_{j}=\frac{\hbar}{\ell}\left(\lambda_{N+1-j}+\left(j-\frac{N+1}{2}\right) \beta\right), \quad j=1, \ldots, N,
$$

\footnotetext{
${ }^{16}$ Quasi-momenta $p_{j}$ have the physical dimension of momentum.
} 
the formulas for the energy and total momentum can be written as

$$
E=\frac{1}{2} \sum_{j=1}^{N} p_{j}^{2}, \quad P=\sum_{j=1}^{N} p_{j},
$$

where they obviously coincide with the sums of energies and momenta of free non-relativistic particles with momenta $p_{j}$. For $p_{j}$ a selection rule applies, namely, $p_{j+1}-p_{j} \geq \gamma / \ell$. The formulae for $E$ and $P$ hint on the degeneracy of the spectrum, as the same values of of these integrals can be realised by different configurations of $p_{j}$. It turns out that the eigenstates of the hamiltonian are separated by the values of higher commuting charges.

Sekiguchi operators. The commuting integrals of the model are given by (3.3). Conjugating these integrals with the ground state wave function, we get

$$
\mathscr{D}_{k}=\Delta^{-1} D_{k} \Delta \text {. }
$$

These new mutually commuting differential operators are known as Sekiguchi operators. They were introduced in [18] as a one-parametric deformation of the generators of the algebra of the invariant differential operators on symmetric spaces.

In the following it is convenient to introduce the following generating functions for $D_{k}$ and $\mathscr{D}_{k}$

$$
D(\zeta)=\sum_{k=0}^{N} \zeta^{N-k}(-1)^{k} D_{k}, \quad \mathscr{D}(\zeta)=\sum_{k=0}^{N} \zeta^{N-k}(-1)^{k} \mathscr{D}_{k},
$$

so that $\mathscr{D}(\zeta)=\triangle^{-1} D(\zeta) \triangle$. Recall that $D(\zeta)$ coincides with determinant (3.5). According to [18], the function $\mathscr{D}(\zeta)$ has the following explicit form

$$
\mathscr{D}(\zeta)=\frac{1}{\prod_{i<j}^{N} Q_{i j}} \sum_{\sigma \in \mathfrak{S}_{N}}(-1)^{\sigma} \prod_{i=1}^{N} Q_{i}^{N-\sigma(i)}\left(\zeta+\frac{\gamma}{\ell}\left(\sigma(i)-\frac{N+1}{2}\right)+i \hbar \partial_{i}\right),
$$

where $\mathfrak{S}_{N}$ is the symmetric group. The Jack polynomials diagonalise not only the momentum and the hamiltonian, but also all the Sekiguchi operators, namely,

$$
\mathscr{D}(\zeta) \cdot J_{\lambda}(Q ; \beta)=\mathbb{Q}(\lambda) \cdot J_{\lambda}(Q ; \beta),
$$

where the eigenvalue is

$$
Q(\zeta)=\prod_{j=1}^{N}\left(\zeta-\frac{\hbar}{\ell}\left(\lambda_{j}-\left(j-\frac{N+1}{2}\right) \beta\right)\right) .
$$

Upon shifting the index $j \rightarrow N+1-j$, the expression for $\mathbb{Q}(\zeta)$ can be rewritten in terms of the quasi-momenta (3.21) as

$$
Q(\zeta)=\prod_{j=1}^{N}\left(\zeta-p_{j}\right)
$$

The polynomial $Q(\zeta)$ is an example of a Baxter polynomial. Its roots $p_{j}$ coincide with the allowed values of quasi-momentum. 


\section{Quantum integrability II: Scattering and Bethe Ansatz}

\subsection{Scattering in classical integrable models}

We start with scattering in classical mechanics. The simplest situation corresponds to a single non-relativistic particle scattering elastically off a fixed target modelled by a potential with a finite interaction range. Excluding from consideration bounded orbits which might exist for attractive potentials, far away from an interaction region asymptotic trajectories are straight lines.

$$
q(t) \rightarrow q_{\text {out }}(t) \equiv q^{\mp}+p^{\mp} t, \quad t \rightarrow \mp \infty,
$$

where coordinates $q^{ \pm}$and momenta $p^{ \pm}=p( \pm \infty)$ constitute the scattering data and a transformation from $\left(q^{-}, p^{-}\right)$to $\left(q^{+}, p^{+}\right)$defines a classical scattering operator, also known as classical $S$-matrix. Scattering of several particles interacting via an admissible (see below) pair-wise potential is considered in a similar manner.

To understand what is special about scattering processes in an integrable model, we consider the example of $N$ particles of equal mass $m=1$ governed by an integrable hamiltonian of the type (1.52) with an admissible potential $v$ that is symmetric $v(q)=v(-q)$, repulsive and impenetrable, and falls off sufficiently rapidly with the distance between particles, to guarantee the existence of an asymptotic region. Examples satisfying these requirements are provided by rational or hyperbolic CMS models, Classical integrability for these models follows from the existence of the Lax representation with the matrix $L$ given by

$$
L=\sum_{j=1}^{N} p_{j} E_{j j}+\sum_{i<j}^{N} u\left(q_{i j}\right) E_{i j}
$$

with an appropriate function $u(q)$.

Non-diffractive scattering. Since the potential is repulsive and impenetrable, particles cannot overtake each other and one can label them according to the order

$$
q_{1}(t)<q_{2}(t)<\ldots<q_{N}(t) .
$$

Since these inequalities are valid for any $t$, from asymptotic conditions (4.1) one obtains the following orderings of the asymptotic momenta

$$
\begin{aligned}
& p_{1}^{-}>p_{2}^{-}>\ldots>p_{N}^{-}, \\
& p_{1}^{+}<p_{2}^{+}<\ldots<p_{N}^{+} .
\end{aligned}
$$

Since $\left|q_{i}-q_{j}\right|=\mathcal{O}(t)$ as $t \rightarrow \pm \infty$, the Lax matrix in the limits $t \rightarrow \pm \infty$ reduces to a diagonal matrix that supports the set of eigenvalues $\lambda_{j}$ of $L$. These eigenvalues are integrals of motion, and if we set $p_{j}^{-}=\lambda_{j}$ then,

$$
\lambda_{1}>\lambda_{2}>\ldots>\lambda_{N} .
$$

Obviously, the same order of eigenvalues must be found at $t=+\infty$, which is only possible if $L(+\infty)$ has the same eigenvalues as $L(-\infty)$ but in the reversed order:

$$
L(-\infty)=\operatorname{diag}\left(p_{1}^{-}, \ldots, p_{N}^{-}\right), \quad L(+\infty)=\operatorname{diag}\left(p_{N}^{+}, \ldots, p_{1}^{+}\right) .
$$


This leads to a simple relation between the scattering data:

$$
p_{N+1-j}^{+}=p_{j}^{-} \text {. }
$$

Thus, the set of incoming asymptotic momenta $\left\{p_{i}^{-}\right\}$coincides with the set of outgoing ones $\left\{p_{i}^{+}\right\}$. This fundamental result is usually referred to as conservation of asymptotic momenta and the corresponding scattering process is described as non-diffractive. Relations (4.4) are independent of the value of the coupling constant that governs the strength of the potential.

Classical phase shift. Due to the coincidence of the sets of incoming and outgoing momenta, we can reinterpret the scattering picture in a different way. Namely, we can associate to each particle a unique asymptotic momentum and assume that the order of particles is the same as that of their momenta. In particular, before scattering the fastest particle is the most left one, and after the scattering it reappears on the right of all the others, as if interactions would be completely absent. This is the so-called transmission representation of scattering in comparison to the reflection representation we started with. From the transmission point of view, individual particles always keep their asymptotic momenta, while scattering shows up in the discontinuity $\delta_{j}$ of the asymptotic coordinates

$$
\begin{aligned}
\delta_{j} & =q_{N+1-j}^{+}-q_{j}^{-} \\
& =\lim _{t \rightarrow+\infty}\left(q_{N+1-j}(t)-q_{j}(-t)-2 p_{j}^{-} t\right) .
\end{aligned}
$$

The quantity $\delta_{j}$, also known as the classical phase shift, completely characterises the scattering process: it shows how much the $j$ th particle has advanced in comparison to a freely moving particle with momentum $p_{j}$. The simplest case is the two-body problem, when one considers scattering of two particles giving rise to the corresponding phase shifts $\delta_{1}$ and $\delta_{2}$. In this case there is a general formula for the two-body phase shift $\delta_{1} \equiv \delta(k)$, where $k=p_{1}^{-}-p_{2}^{-}>0$, which expresses the shift via the potential [19]

$$
\delta(k)=x_{0}(k)-\int_{x_{0}(k)}^{\infty} \mathrm{d} x\left(\frac{1}{\sqrt{1-\frac{4 v(x)}{k^{2}}}}-1\right) .
$$

Here $x_{0}=x_{0}(k)$ is found from $4 v\left(x_{0}\right)=k^{2}$. The shift depends on the difference $k$ of asymptotic momenta but not on the asymptotic coordinates $q_{i}^{-}$. It also follows from (4.6) that $\delta(k)$ is an even function of $k$. The detailed derivation of (4.6) can be found in [9]. As discussed in this book, for the rational and hyperbolic CMS models the corresponding phase shifts are

$$
\begin{aligned}
& \delta_{1}=0=-\delta_{2} \quad \text { (rational), } \\
& \delta_{1}=\log \left(1+\frac{\gamma^{2}}{k^{2}}\right)=-\delta_{2} \quad \text { (hyperbolic), }
\end{aligned}
$$

If we restore the physical dimensions and the dependence on $\ell$, the answer for the hyperbolic case $\delta(k) \equiv \delta_{1}$ reads as

$$
\delta(k)=\ell \log \left(1+\frac{\gamma^{2}}{k^{2} \ell^{2}}\right)
$$


Now we can discuss $\delta_{j}$ for the case of many particles.

Factorisation of the classical S-matrix Recall that the hamiltonian of a dynamical system gives rise to a one-parametric group $U_{t}$ of canonical transformations with time $t$ playing the role of parameter. For any function $f(p, q)$ on the phase space its time evolution $f(p, q, t) \equiv f(p(t), q(t))$ is given by

$$
f(p, q, t)=\left(U_{t} f\right)(p, q)=e^{t\{H, \cdot\}} \circ f(p, q) .
$$

We assuming the existence of in- and out-asymptotics, namely,

$$
e^{t\{H, \cdot\}} \circ\left(p_{i}, q_{i}\right) \rightarrow\left(p_{i}^{ \pm}, p_{i}^{ \pm} t+q_{i}^{ \pm}\right), \quad t \rightarrow \pm \infty,
$$

where the variables $\left(p_{i}^{ \pm}, q_{i}^{ \pm}\right)$define the asymptotic phase spaces

$$
\mathscr{P}^{ \pm}=\left\{\left(p^{ \pm}, q^{ \pm}\right) \in \mathbb{R}^{2 N}, p_{1}^{ \pm} \lessgtr p_{2}^{ \pm} \lessgtr \ldots \lessgtr p_{N}^{ \pm}\right\} .
$$

Since the Poisson brackets between canonical variables are preserved under the time evolution, the asymptotic data $\left(p_{i}^{-}, q_{i}^{-}\right)$and $\left(q_{i}^{+}, p_{i}^{+}\right)$also form canonical pairs, i.e. the asymptotic spaces are symplectic. This allows one to define the classical analogue of the quantum-mechanical wave operators $\Omega_{ \pm}: \mathscr{P}^{\mp} \rightarrow \mathscr{P}$,

$$
\Omega_{ \pm}=\lim _{t \rightarrow \mp \infty} e^{-t\{H, \cdot\}} \circ e^{t\left\{H_{0}^{\mp}, \cdot\right\}}
$$

where $H_{0}^{ \pm}=\frac{1}{2} \sum_{j=1}^{N}\left(p_{j}^{ \pm}\right)^{2}$ are free hamiltonians. The wave maps are canonical and they are used to construct the classical S-matrix

$$
S_{\text {class }}=\Omega_{-}^{-1} \Omega_{+}: \mathscr{P}^{-} \rightarrow \mathscr{P}^{+},
$$

which is also a canonical transformation.

Phase shifts $\delta_{j}$ for many-body scattering can be found by relying on canonicity of the asymptotic phase spaces and the relations (4.4). One has

$$
\delta_{i j}=\left\{p_{N+1-i}^{+}, q_{N+1-j}^{+}\right\}=\left\{p_{i}^{-}, q_{j}^{-}+\delta^{j}\right\}=\delta_{i j}+\frac{\partial \delta_{j}}{\partial q_{i}^{-}} .
$$

Hence, $\partial \delta_{j} / \partial q_{i}^{-}=0$, i.e. $\delta_{j}$ does not depends on $q^{-}$and, therefore, it can be computed by arranging the asymptotic data $q_{1}^{-}, \ldots, q_{N}^{-}$into a special configuration such that collisions take place pairwise, with asymptotically large times in between of any two subsequent collisions. This shows that the multi-body phase shift can be found by simply summing up the two-body phase shifts arising from collisions of $j$ 's particle with the rest

$$
\delta_{j}=q_{N-j+1}^{+}-q_{j}^{-}=\underbrace{\sum_{k>j} \delta\left(p_{j}^{-}-p_{k}^{-}\right)}_{\text {right particles }}-\underbrace{\sum_{k<j} \delta\left(p_{j}^{-}-p_{k}^{-}\right)}_{\text {left particles }},
$$

see Fig. 2 which describes the corresponding kinematic configurations before and after scattering. This result (4.13) is a consequence of the existence of a complete set of integrals of motion 


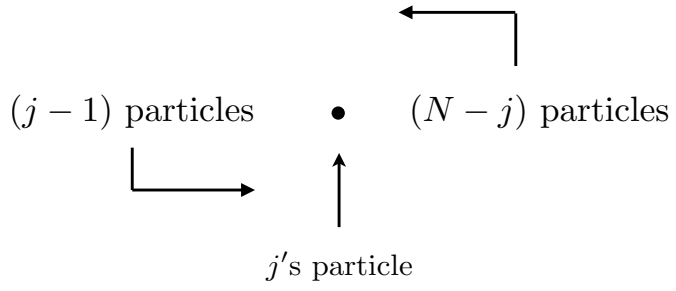

Before scattering

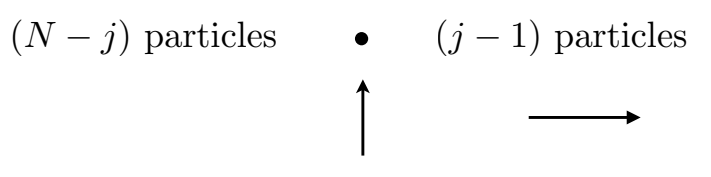

$j^{\prime}$ s particle

After scattering

Figure 2: Rearrangement of particles in the scattering process.

responsible for (4.4). There are no separate three- and higher-body events and the multi-particle scattering process is completely characterised by the two-body phase shift.

To determine the classical S-matrix, we consider the generating function $\Phi\left(q^{-}, p^{+}\right)$of the canonical transformation $\left(q_{i}^{-}, p_{i}^{-}\right) \rightarrow\left(q_{i}^{+}, p_{i}^{+}\right)$

$$
p_{i}^{-}=\frac{\partial \Phi\left(q^{-}, p^{+}\right)}{\partial q_{i}^{-}}=p_{N+1-i}^{+}, \quad q_{i}^{+}=\frac{\partial \Phi\left(q^{-}, p^{+}\right)}{\partial p_{i}^{+}}=q_{N+1-i}^{-}+\delta_{N+1-i},
$$

where, according to (4.13),

$$
\delta_{N+1-i}=\sum_{k<i} \delta\left(p_{i}^{+}-p_{k}^{+}\right)-\sum_{k>i} \delta\left(p_{i}^{+}-p_{k}^{+}\right) .
$$

Integrating (4.14), one gets

$$
\Phi\left(q^{-}, p^{+}\right)=\sum_{i=1}^{N} q_{i}^{-} p_{N+1-i}^{+}+\sum_{i<j}^{N} \theta\left(p_{i}^{+}-p_{j}^{+}\right),
$$

where $\theta(k)$ is an integrated phase shift

$$
\theta(k)=k x_{0}(k)-k \int_{x_{0}(k)}^{\infty} \mathrm{d} x\left(\sqrt{1-\frac{4 v(x)}{k^{2}}}-1\right), \quad \frac{\partial \theta}{\partial k}=\delta(k) .
$$

According to this formula, $\theta(k)$ is an odd function of $k$. Were the theory free, the relation between $\mathscr{P}^{+}$and $\mathscr{P}^{-}$would reduce to relabelling of particles described by the generating function $\Phi_{0}\left(q^{-}, p^{+}\right)=\sum_{i=1}^{N} q_{i}^{-} p_{N+1-i}^{+}$. The non-trivial part of the generating function is, therefore, the classical S-matrix

$$
S_{\mathrm{cl}}=\sum_{i<j}^{N} \theta\left(p_{i}^{+}-p_{j}^{+}\right)
$$

From the point of view of the correspondence between classical and quantum mechanics, this Smatrix is the leading term in the semi-classical expansion of the phase $\theta$ of the quantum-mechanical 
wave function $\Psi=a e^{\frac{i}{\hbar} \theta}$ in powers of $\hbar$. The fact that this S-matrix factorises into the sum of its elementary two-body phase shifts is a deep consequence of integrability and it persists in the quantum theory as well.

\subsection{Factorisation of scattering matrix}

Here we consider the scattering problem in quantum mechanics. Our starting point is the Schrödinger equation for the multi-body wave function $\Psi\left(q_{1}, \ldots, q_{N}\right)$

$$
-\frac{1}{2 m} \sum_{i=1}^{N} \frac{\partial^{2}}{\partial q_{i}^{2}} \Psi\left(q_{1}, \ldots, q_{N}\right)+\sum_{i \neq j} v\left(q_{i}-q_{j}\right) \Psi\left(q_{1}, \ldots, q_{N}\right)=E \Psi\left(q_{1}, \ldots, q_{N}\right) .
$$

The potential $v$ is taken to be admissible and translation invariant, thereby the total momentum $P$ is conserved; $\hbar=1$. We also assume that particles are distinguishable, so that the wave function does not have any particular symmetry under permutations of coordinates.

Bethe wave function. If a quantum model is integrable, we can search for the wave function as a common eigenstate of a family ${ }^{17}$ of $N$ pair-wise commuting operators $H_{k}$

$$
H_{k} \Psi\left(q_{1}, \ldots, q_{N}\right)=h_{k} \Psi\left(q_{1}, \ldots, q_{N}\right), \quad k=1, \ldots, N .
$$

When separation between any two neighbouring particles is large, the potential terms in $H_{k}$ are negligibly small and can be ignored, so that $H_{k}$ turn into the conservation laws $H_{k}^{(0)}$ of the free theory

$$
H_{k}^{(0)}=\sum_{j}\left(-i \partial_{j}\right)^{k}
$$

Considering a kinematic domain (sector) where particles are far apart according to the pattern

$$
q_{1}<q_{2}<\ldots<q_{N}
$$

a common eigenstate of the free problem $H_{k}^{(0)} \Psi=h_{k} \Psi$ is elementary to guess

$$
\Psi \sim e^{i p_{1} q_{1}+\ldots i p_{N} q_{N}} .
$$

Here the numbers $p_{j}$ are asymptotic momenta which satisfy a system of $N$ polynomial equations

$$
\sum_{j=1}^{N} p_{j}^{k}=h_{k}
$$

In particular, the energy and momentum are

$$
E=\frac{1}{2} \sum_{j=1}^{N} p_{j}^{2}, \quad P=\sum_{j=1}^{N} p_{j}
$$

We assume that for given $h_{k}$ this system has a unique solution where momenta $p_{j}$ respect the ordering

$$
p_{1}>p_{2}>\ldots>p_{N} .
$$

\footnotetext{
${ }^{17}$ We assume that the hamiltonian $H$ and the total momentum $P$ belong to this family.
} 
Then any other solution of (4.22) will be obtained by permutations of the set (4.24). This implies that the general solution of the free theory in the kinematic domain (4.20) will be given by a superposition of free waves characteristic to solutions of scattering type

$$
\Psi\left(q_{1}, \ldots, q_{N}\right)=\sum_{\tau \in \mathfrak{S}_{N}} \mathscr{A}(\tau) e^{i q_{1} p_{\tau(1)}+\ldots+i q_{N} p_{\tau(N)}},
$$

where the sum runs over permutations $\tau$ from the symmetric group $\mathfrak{S}_{N}$. For the asymptotic expression (4.25) the amplitudes $\mathscr{A}(\tau)$ are arbitrary.

In general, the configuration space $\mathbb{R}^{N}$ can be divided into $N$ ! disconnected sectors, each sector corresponds to a certain ordering of coordinates

$$
q_{\sigma(1)}<q_{\sigma(2)}<\ldots<q_{\sigma(N)},
$$

where the latter are labelled by permutations $\sigma \in \mathfrak{S}_{N}$. The set $\left\{p_{j}\right\}$ of asymptotic momenta must be the one and the same for any asymptotic domain because the spectral invariants $h_{k}$ are globally defined. The asymptotic wave function in the $\sigma$-sector (4.26) is a superposition of plane waves

$$
\Psi\left(q_{1}, \ldots, q_{N} \mid \sigma\right)=\sum_{\tau \in \mathfrak{S}_{N}} \mathscr{A}(\sigma \mid \tau) e^{i q_{\sigma(1)} p_{\tau(1)}+\ldots+i q_{\sigma(N)} p_{\tau(N)},}
$$

where the complex amplitudes $\mathscr{A}(\sigma \mid \tau)$ are combined into a $N ! \times N !$ matrix with undetermined coefficients. To deal with all sectors at once, it is convenient to introduce the following function

$$
\Theta\left(q_{\sigma(1)}<\ldots<q_{\sigma(N)}\right)=\prod_{i=1}^{N-1} \Theta\left(q_{\sigma(i+1)}-q_{\sigma(i)}\right),
$$

where $\Theta(x)$ is the Heaviside $\Theta$-function. Multiplying (4.27) with (4.28) to account for the sector restrictions, we then sum over all sectors to get

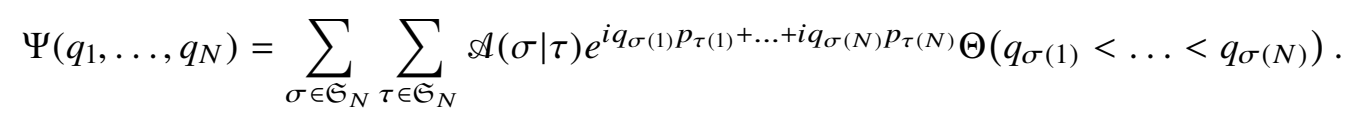

The expression (4.29) is called the Bethe wave function and it was introduced by C. N. Yang [20] .

The Bethe wave function describes an asymptotics of the multi-body wave function in integrable models. The fact that any asymptotic wave is determined by the same set of momenta, up to permutations, means that the scattering process is non-diffractive. Three- and higher-body events that would lead to a continuous redistribution of momenta are prohibited by a sufficiently large number of conservation laws. The coefficients $\mathscr{A}(\sigma \mid \tau)$ should be recovered by considering asymptotics of the exact solution of (4.18). Fortunately, there is a way to find these coefficients from the asymptotic data of the two-body problem, which brings to light the concept of S-matrix.

S-matrix. Consider two sectors which differ only by the order of two neighbouring particles $q_{i}$ and $q_{j}$, so that in the first sector $q_{i}<q_{j}$ and $q_{i}>q_{j}$ in the second. These sectors are neighbours and have the hyperplane $q_{i}=q_{j}$ as a common boundary. Extrapolation of the wave function through this boundary can always be done by keeping all the other coordinates far away from $q_{i} \approx q_{j}$ and from each other. Physically, this extrapolation corresponds to a two-body scattering event. Starting from any sector, one can obviously reach any other by passing through the adjacent sectorial boundaries, 
albeit not in a unique way. The neighbouring sectors are thus connected by simple transpositions $\alpha_{j} \in \mathfrak{S}_{N}, j=1, \ldots, N-1$. The relation between the corresponding amplitudes can be described as follows. We introduce a column vector $\Phi(\tau)$ which comprises the amplitudes in all the sectors corresponding to the same permutation $\tau$ of momenta

$$
\Phi(\tau) \equiv\left\{\mathscr{A}(\sigma \mid \tau), \sigma \in \mathfrak{S}_{N}\right\}
$$

The following connection formula that expresses the scattering of two neighbouring particles at $q_{j}$ and $q_{j+1}$ particles with momenta $p_{\tau(j)}$ and $p_{\tau(j+1)}$ is

$$
\Phi\left(\alpha_{j} \tau\right)=Y_{j}\left(p_{\tau(j)}, p_{\tau(j+1)}\right) \Phi(\tau)
$$

where we introduced Yang's scattering operators $Y_{j}$

$$
Y_{j}\left(p_{1}, p_{2}\right)=A\left(p_{1}, p_{2}\right) \mathbb{1}+B\left(p_{1}, p_{2}\right) \pi\left(\alpha_{j}\right),
$$

where $j=1, \ldots, N-1$. Here $\pi$ is the left regular representation of $\mathfrak{S}_{N}$, the functions $A$ and $B$ are the reflection and transmission coefficients of the two-body scattering problem. Yang's operator $Y_{j}$ is a $N ! \times N$ ! matrix which acts on the vector $\Phi(\tau)$ by the usual matrix multiplication. Equation (4.31) can be called a connection formula as it can be used to connect any two sectorial amplitudes and eventually to express them all via $\Phi(e)$, where $e$ is the identity permutation. For the proof of (4.31) the reader can consult, for instance, [9].

More generally, one introduces the two-body S-matrices

$$
S_{i j}\left(p_{i}, p_{j}\right)=B\left(p_{i}, p_{j}\right) \mathbb{1}+A\left(p_{i}, p_{j}\right) \pi\left(\alpha_{i j}\right)
$$

where $\alpha_{i j}$ is a transposition, in particular, $\alpha_{i} \equiv \alpha_{i i+1}$. The relation between Yang's operators and the S-matrices is $Y_{j}=\pi\left(\alpha_{j}\right) S_{j j+1}$. Further we note that the defining relations of the symmetric group impose non-trivial conditions on Yang's operators and on S-matrices. In particular, the S-matrix must satisfy

$$
S_{12}\left(p_{1}, p_{2}\right) S_{21}\left(p_{2}, p_{1}\right)=\mathbb{1}
$$

and

$$
S_{12}\left(p_{1}, p_{2}\right) S_{13}\left(p_{1}, p_{3}\right) S_{23}\left(p_{2}, p_{3}\right)=S_{23}\left(p_{2}, p_{3}\right) S_{13}\left(p_{1}, p_{3}\right) S_{12}\left(p_{1}, p_{2}\right) .
$$

The last relation is the Yang-Baxter equation for the two-body S-matrix. It expresses the equivalence of two different ways to factorise a three-body S-matrix $S_{123}$ into a product of two-body S-matrices. Thus, integrability implies consistent factorisation of scattering process and the corresponding $S$ matrix in a sequence of two-body events and S-matrices, giving rise to the notion of Factorised Scattering Theory [21].

Thus, combing ansatz (4.28) with the description of scattering in terms of transmission and reflection coefficients allows one to determine all the amplitudes in (4.28) via $\Phi(e)$. The amplitude column $\Phi(e)$ remains undetermined which reflects the absence of symmetry of the wave function for distinguishable particles. For the ultimate determination of the transmission and reflection 
coefficients one needs to solve the Schrödinger equation for two particles, the latter being the onedimensional problem due to translation invariance. Below we illustrate the concept of the Bethe wave function and computation of the reflection and transmission coefficients on two examples: the Lieb-Liniger model for distinguishable particles (the delta-interaction model) and the CMS models (rational and hyperbolic).

Lieb-Liniger model for distinguishable particles. As an explicit realisation of the Bethe wave function construction above, we consider the two-body problem for the Lieb-Liniger model described by the Hamiltonian (1.54). The Bethe wave function (4.29) for the two-particle case reads as

$$
\begin{aligned}
\Psi\left(q_{1}, q_{2}\right) & =\Theta\left(q_{1}<q_{2}\right)\left\{\mathscr{A}(12 \mid 12) e^{i\left(p_{1} q_{1}+p_{2} q_{2}\right)}+\mathscr{A}(12 \mid 21) e^{i\left(p_{2} q_{1}+p_{1} q_{2}\right)}\right\} \\
& +\Theta\left(q_{2}<q_{1}\right)\left\{\mathscr{A}(21 \mid 12) e^{i\left(p_{1} q_{2}+p_{2} q_{1}\right)}+\mathscr{A}(21 \mid 21) e^{i\left(p_{2} q_{2}+p_{1} q_{1}\right)}\right\} .
\end{aligned}
$$

Thus, we have four amplitudes involved. Separating the center of mass

$$
q=q_{1}-q_{2}, \quad Q=q_{1}+q_{2},
$$

so that

$$
q_{1}=\frac{Q+q}{2}, \quad q_{2}=\frac{Q-q}{2},
$$

we get

$$
\Psi\left(q_{1}, q_{2}\right)=e^{\frac{i}{2}\left(p_{1}+p_{2}\right) Q} \psi(q),
$$

where

$$
\begin{aligned}
\psi(q) & =\theta(-q)\left\{\mathscr{A}(12 \mid 12) e^{\frac{i}{2}\left(p_{1}-p_{2}\right) q}+\mathscr{A}(12 \mid 21) e^{-\frac{i}{2}\left(p_{1}-p_{2}\right) q}\right\} \\
& +\theta(q)\left\{\mathscr{A}(21 \mid 12) e^{-\frac{i}{2}\left(p_{1}-p_{2}\right) q}+\mathscr{A}(21 \mid 21) e^{\frac{i}{2}\left(p_{1}-p_{2}\right) q}\right\} .
\end{aligned}
$$

Continuity of $\psi(q)$ at 0 requires

$$
\mathscr{A}(12 \mid 12)+\mathscr{A}(12 \mid 21)=\mathscr{A}(21 \mid 21)+\mathscr{A}(21 \mid 21) .
$$

The left and right derivatives at zero are

$$
\begin{aligned}
& \psi^{\prime}(+0)=-\frac{i}{2}\left(p_{1}-p_{2}\right) \mathscr{A}(21 \mid 12)+\frac{i}{2}\left(p_{1}-p_{2}\right) \mathcal{A}(21 \mid 21), \\
& \psi^{\prime}(-0)=\frac{i}{2}\left(p_{1}-p_{2}\right) \mathscr{A}(12 \mid 12)-\frac{i}{2}\left(p_{1}-p_{2}\right) \mathcal{A}(12 \mid 21) .
\end{aligned}
$$

The wave function for the relative motion is the subject of the following Schrödinger equation

$$
-\psi^{\prime \prime}(q)+v(q) \psi(q)=\frac{k^{2}}{4} \psi(q), \quad k=p_{1}-p_{2} .
$$

For the case at hand the potential is $v(q)=\kappa \delta(q)$ and we get

$$
-\psi^{\prime \prime}(q)+\kappa \delta(q) \psi(q)=\frac{k^{2}}{4} \psi(q) .
$$


We then integrate both sides of equation (4.41) over a small segment $[-\epsilon, \epsilon]$ and then send $\epsilon \rightarrow 0$. Due to continuity of the wave function, we get an equation for the discontinuity of its derivative at the origin

$$
\psi^{\prime}(+0)-\psi^{\prime}(-0)=\kappa \psi(0)
$$

Substituting here the derivatives found above, we get

$$
\begin{array}{r}
\frac{i}{2}\left(p_{1}-p_{2}\right)\{-\mathscr{A}(21 \mid 12)+\mathscr{A}(21 \mid 21)-\mathscr{A}(12 \mid 12)+\mathscr{A}(12 \mid 21)\} \\
=\kappa\{\mathscr{A}(12 \mid 12)+\mathscr{A}(12 \mid 21)\} .
\end{array}
$$

By using (4.39) we first remove from this equation $\mathscr{A}(21 \mid 21)$

$$
\begin{array}{r}
\frac{i}{2}\left(p_{1}-p_{2}\right)\{-\mathscr{A}(21 \mid 12)+\mathscr{A}(12 \mid 12)+\mathscr{A}(12 \mid 21)-\mathscr{A}(21 \mid 12)-\mathscr{A}(12 \mid 12)+\mathscr{A}(12 \mid 21)\} \\
=\kappa\{\mathscr{A}(12 \mid 12)+\mathscr{A}(12 \mid 21)\},
\end{array}
$$

so that

$$
\mathscr{A}(12 \mid 21)-\mathscr{A}(21 \mid 12)=-x_{12}\{\mathscr{A}(12 \mid 12)+\mathscr{A}(12 \mid 21) \mathscr{A}\}
$$

where we have introduced

$$
x_{12}=\frac{i \kappa}{p_{1}-p_{2}} .
$$

The last equation can be solved for $\mathscr{A}(12 \mid 21)$

$$
\mathscr{A}(12 \mid 21)=-\frac{x_{12}}{1+x_{12}} \mathscr{A}(12 \mid 12)+\frac{1}{1+x_{12}} \mathscr{A}(21 \mid 12) .
$$

Now we look at (4.43) again and remove this time $\mathscr{A}(12 \mid 21)$ by using (4.39)

$$
\begin{array}{r}
\frac{i}{2}\left(p_{1}-p_{2}\right)\{-\mathscr{A}(21+12)+\mathscr{A}(21 \mid 21)-\mathscr{A}(12 \mid 12)+\mathscr{A}(21 \mid 12)+\mathscr{A}(21 \mid 21)-\mathscr{A}(12 \mid 12)\} \\
=\kappa\{\mathscr{A}(21 \mid 21)+\mathscr{A}(21 \mid 21)\} .
\end{array}
$$

This gives

$$
\mathscr{A}(21 \mid 21)=-\frac{x_{12}}{1+x_{12}} \mathscr{A}(21 \mid 12)+\frac{1}{1+x_{12}} \mathscr{A}(12 \mid 12) .
$$

Equations (4.46) and (4.47) can be compactly written as

$$
\Phi(21)=Y \Phi(12), \quad Y=-\frac{x_{12}}{1+x_{12}} \mathbb{1}+\frac{1}{1+x_{12}} \pi_{12}, \quad \pi_{12}=\left(\begin{array}{ll}
0 & 1 \\
1 & 0
\end{array}\right),
$$

where $Y \equiv Y_{1}$ and $\Phi(\tau)$ are columns of $\mathscr{A}(\sigma \mid \tau)$, namely,

$$
\Phi(12)=\left(\begin{array}{l}
\mathscr{A}(12 \mid 12) \\
\mathscr{A}(21 \mid 12)
\end{array}\right), \quad \Phi(21)=\left(\begin{array}{l}
\mathscr{A}(12 \mid 21) \\
\mathscr{A}(21 \mid 21)
\end{array}\right) .
$$


Comparing (4.48) to the general form (4.32), we read off the reflection and transmission coefficients for the Lieb-Liniger model

$$
A=-\frac{i \kappa}{p_{1}-p_{2}+i \kappa}, \quad B=\frac{p_{1}-p_{2}}{p_{1}-p_{2}+i \kappa} .
$$

The vector $\Phi(21)$ is fully determined by $\Phi(12)$, on the other hand $\Phi(12)=\Phi(e)$ remains arbitrary, i.e. amplitudes in different sectors remain unrelated.

What is remarkable is that with the ansatz (4.36) and coefficients (4.50) we were able to obtain the exact rather than asymptotic solution of the Schrödinger equation. This phenomenon happens due to the extremely short-range behaviour of the potential and therefore is peculiar to this integrable model.

Hyperbolic CMS model. Writing the two-body wave function of the hyperbolic CMS model as (4.37), for $\psi(q)$ we have the following Schrödinger equation $(\ell=1)$

$$
-\psi^{\prime \prime}(q)+\frac{\gamma(\gamma-1)}{4 \sinh ^{2} \frac{1}{2} q} \psi(x)=\frac{k^{2}}{4} \psi(q) .
$$

The potential is repulsive for $\beta>1$. Since it is also impenetrable we are looking for the solution that vanishes at $q=0$. We can thus solve the problem for $q>0$ with the condition $\psi(0)=0$ and then extend the solution for negative $q$ in two possible ways $\psi(-q)= \pm \psi(q)$ corresponding to the symmetric or anti-symmetric wave function. For the symmetric wave function we deal with hard core bosons and for the anti-symmetric one with usual fermions. The solution for $q>0$ which satisfies the required boundary condition reads

$$
\begin{aligned}
\psi(q)= & \left(e^{q / 2}-e^{-q / 2}\right)^{-i k}{ }_{2} F_{1}\left(\gamma+i k, 1-\gamma+i k, 1+i k ; \frac{1}{1-e^{q}}\right) \\
& -e^{i k q / 2} \frac{\Gamma(1+i k) \Gamma(\gamma-i k)}{\Gamma(1-i k) \Gamma(\gamma+i k)}{ }_{2} F_{1}\left(\gamma, 1-\gamma, 1-i k ; \frac{1}{1-e^{q}}\right),
\end{aligned}
$$

where ${ }_{2} F_{1}(a, b, c ; z)$ is the hypergeometric function. In the limit $q \rightarrow+\infty$ one find

$$
\psi(q) \rightarrow e^{-i k q / 2}-\frac{\Gamma(1+i k) \Gamma(\gamma-i k)}{\Gamma(1-i k) \Gamma(\gamma+i k)} e^{i k q / 2} .
$$

Comparing this asymptotics with the second line in (4.38), we get

$$
\mathscr{A}(21 \mid 12)=1, \quad \mathscr{A}(21 \mid 21)=-\frac{\Gamma(1+i k) \Gamma(\gamma-i k)}{\Gamma(1-i k) \Gamma(\gamma+i k)} .
$$

We further have $\mathscr{A}(12 \mid 12)= \pm \mathscr{A}(21 \mid 12)$ and $\mathscr{A}(12 \mid 21)= \pm \mathscr{A}(21 \mid 21)$, where signs " + " and " - " correspond to the symmetric and anti-symmetric wave functions, respectively. From (4.49) for both symmetric and anti-symmetric wave functions, one concludes that

$$
\Phi(21)=\frac{\mathscr{A}(21 \mid 21)}{\mathscr{A}(21 \mid 12)} \Phi(12),
$$

so that there is non-trivial reflection coefficient, which is a pure phase,

$$
A=-\frac{\Gamma(1+i k) \Gamma(\gamma-i k)}{\Gamma(1-i k) \Gamma(\gamma+i k)}=-e^{-i \theta},
$$


while the transmission coefficient $B$ vanishes. Restoring all the parameters by substitutions $\gamma \rightarrow$ $\gamma / \hbar, k \rightarrow k \rightarrow k \ell / \hbar, \theta \rightarrow \theta / \hbar$, we obtain for the scattering phase

$$
\theta=i \hbar \log \left[\frac{\Gamma\left(1+i \frac{k \ell}{\hbar}\right) \Gamma\left(\frac{\gamma}{\hbar}-i \frac{k \ell}{\hbar}\right)}{\Gamma\left(1-i \frac{k \ell}{\hbar}\right) \Gamma\left(\frac{\gamma}{\hbar}+i \frac{k \ell}{\hbar}\right)}\right] .
$$

There are two interesting limiting cases of this formula. Firstly, one can consider the rational limit $\ell \rightarrow \infty$. In this limit one gets $\theta=\pi(\gamma-\hbar) \operatorname{sign}(k)$. From here $\delta(k)=\theta^{\prime}(k)=\pi(\gamma-\hbar) \delta(k)$ which vanishes for $k \neq 0$, i.e. we observe the same result as for the classical theory where the phase shift was found to vanish. Secondly, there is a classical limit corresponding to taking the action variable $k \ell$ to be large in comparison to $\hbar$. In this limit on finds

$$
\theta=k \ell \log \left(1+\frac{\gamma^{2}}{k^{2} \ell^{2}}\right)+2 \gamma \arctan \frac{k \ell}{\gamma}
$$

which is the integrated phase shift corresponding to $\delta(k)$ in (4.8).

Thus, for the hyperbolic (and rational) CMS model the exact two-body wave function (4.52) for intermediate values of its arguments is rather intricate but is well approaching the Bethe form of the wave function in the asymptotic limit.

\subsection{Bethe-Yang equations}

To describe thermodynamics of an integrable model, we need to consider a system of $N$ interacting particles confined in a one-dimensional box. If the size $L$ of the box is large enough one can still well separate particles to apply the concept of the Bethe wave function. However, the new feature is that to account for the box boundaries one needs to subject the wave function to certain boundaries conditions, of which the periodic boundary conditions is the simplest choice. Thus, the wave function $\Psi$ depending on variables $0 \leq q_{i} \leq L$ is now required to satisfy

$$
\Psi\left(q_{1}, \ldots, q_{j}=0, \ldots, q_{N}\right)=\Psi\left(q_{1}, \ldots, q_{j}=L, \ldots, q_{N}\right), \quad \forall j
$$

For the Bethe wave function (4.29) this yields

$$
\begin{aligned}
& \sum_{\substack{\sigma \in \mathfrak{S}_{N} \\
\sigma(1)=j}} \sum_{\tau \in \mathfrak{S}_{N}} \mathcal{A}(\sigma \mid \tau) e^{i \sum_{k=2}^{N} q_{\sigma(k)} p_{\tau(k)}} \Theta\left(q_{\sigma(2)}<\ldots<q_{\sigma(N)}\right) \\
& \quad=\sum_{\substack{\sigma \in \mathfrak{S}_{N} \\
\sigma(N)=j}} \sum_{\tau \in \mathfrak{S}_{N}} \mathcal{A}(\sigma \mid \tau) e^{i L p_{\tau(N)}} e^{i \sum_{k=1}^{N} q_{\sigma(k)} p_{\tau(k)}} \Theta\left(q_{\sigma(1)}<\ldots<q_{\sigma(N-1)}\right) .
\end{aligned}
$$

where on the right hand side in the exponent one has $q_{\sigma(N)}=q_{j}=0$. To be able to compare these expressions, we introduce the cyclic permutation $\xi \in \mathfrak{S}_{N}$ which in the two line notation reads as

$$
\xi=\alpha_{N-1} \ldots \alpha_{1}=\left(\begin{array}{lllcc}
1 & 2 & N-1 & N \\
2 & 3 & \cdots & N & 1
\end{array}\right)
$$


and change on the right hand side of (4.58) the summation variables $\sigma, \tau$ as $\sigma \rightarrow \xi \sigma$ and $\tau \rightarrow \xi \tau$. Since $\sigma$ obeys $\sigma(1)=j$, then $\xi \sigma(N)=\sigma(\xi(N))=\sigma(1)=j$. This yieds

$$
\begin{aligned}
\sum_{\substack{\sigma \in \mathfrak{S}_{N} \\
\sigma(1)=j}} \sum_{\tau \in \mathfrak{S}_{N}} \mathscr{A}(\sigma \mid \tau) e^{i \sum_{k=2}^{N} q_{\sigma(k)} p_{\tau(k)}} \Theta\left(q_{\sigma(2)}<\ldots<q_{\sigma(N)}\right) \\
\quad=\sum_{\substack{\sigma \in \mathfrak{S}_{N} \\
\sigma(1)=j}} \sum_{\tau \in \mathfrak{S}_{N}} \mathcal{A}(\xi \sigma \mid \xi \tau) e^{i L p_{\xi \tau(N)}} e^{i \sum_{k=2}^{N} q_{\sigma(k)} p_{\tau(k)}} \Theta\left(q_{\sigma(2)}<\ldots<q_{\sigma(N)}\right) .
\end{aligned}
$$

Here $p_{\xi \tau(N)}=p_{\tau(\xi(N))}=p_{\tau(1)}$. From (4.60) it follows that

$$
\mathscr{A}(\sigma \mid \tau)=\mathscr{A}(\xi \sigma \mid \xi \tau) e^{i L p_{\tau(1)}} .
$$

This is a requirement on the coefficients of the asymptotic wave function in order for the latter to satisfy periodic boundary conditions. Invoking the left regular representation $\pi$ of $\mathfrak{S}_{N}$, equations (4.61) can be written as conditions for the vector $\Phi(\tau)$ defined in (4.30):

$$
\pi(\xi) \Phi(\tau)=e^{i L p_{\tau(1)}} \Phi(\xi \tau) .
$$

Obviously, (4.62) should be satisfied for any $\tau$. It is convenient to consider a set $\left\{\tau_{j}\right\}_{j=1}^{N}$, where

$$
\tau_{j} \equiv \alpha_{1} \ldots \alpha_{j-1}=\left(\begin{array}{rrrrrrrr}
1 & 2 & 3 & j & j+1 & \ldots \\
j & 1 & 2
\end{array} \ldots \begin{array}{llll}
j-1 & j+1
\end{array} \ldots, \quad \tau_{1}=e,\right.
$$

so that $\xi \tau_{j}=\alpha_{N-1} \ldots \alpha_{j}$. With this choice $\tau(1)=j$ and (4.62) boils down to

$$
\pi\left(\alpha_{N-1} \ldots \alpha_{1}\right) \Phi\left(\alpha_{1} \ldots \alpha_{j-1}\right)=e^{i L p_{j}} \Phi\left(\alpha_{N-1} \ldots \alpha_{j}\right) .
$$

Applying to both sides of this expression the connection formula (4.31), one gets

$$
\begin{aligned}
\pi\left(\alpha_{j-1}\right) & \ldots \pi\left(\alpha_{1}\right) Y_{1}\left(p_{1}, p_{j}\right) \ldots Y_{j-1}\left(p_{j-1}, p_{j}\right) \Phi(e) \\
& =e^{i L p_{j}} \pi\left(\alpha_{j}\right) \ldots \pi\left(\alpha_{N-1}\right) Y_{N-1}\left(p_{j}, p_{N}\right) \ldots Y_{j}\left(p_{j}, p_{j+1}\right) \Phi(e) .
\end{aligned}
$$

Using the definition (4.33) of the two-body S-matrix the last expression can be cast in the form

$$
S_{j+1 j} S_{j+2 j} \ldots S_{N j} \cdot S_{1 j} \ldots S_{j-1 j} \Phi(e)=e^{i L p_{j}} \Phi(e) .
$$

Introduce the following matrices

$$
T_{j}=S_{j+1 j} S_{j+2 j} \ldots S_{N j} \cdot S_{1 j} \ldots S_{j-1 j} .
$$

Equations (4.64) are equivalent to the statement that $|\Phi\rangle \equiv \Phi(e)$ is a common eigenvector of $N$ matrix operators $T_{j}$

$$
T_{j}|\Phi\rangle=\Lambda_{j}|\Phi\rangle,
$$

where $j=1, \ldots, N$. These are matrix Bethe-Yang equations. Once a common eigenvalue, which is a function of momenta, is found, one is left to solve a system of scalar Bethe equations

$$
\Lambda_{j}=e^{i L p_{j}}
$$


to determine the momenta $p_{j}$. Equations (4.67) can be thus considered as quantisation conditions for particle momenta that arise in a large but finite volume.

Compatibility of the system (4.66) requires that matrices $T_{j}$ for various $j$ pair-wise commute. They do indeed so as a consequence of the condition (4.34) and the Yang-Baxter equation (4.35). Demanding the Bethe wave function to be of the symmetry type described by the Young diagram $\lambda$, we should also require the vector $|\Phi\rangle$ to transform in the same representation. Correspondingly, the scattering operators and the operators $T_{j}$ are also restricted to $\lambda$. The problem therefore reduces to solving the system (4.66) for a given irreducible representation of $\mathfrak{S}_{N}$ with a subsequent reconstruction from this solution of the corresponding Bethe wave function.

In the case of scalar S-matrices, where $|\Phi\rangle$ is one-dimensional, the diagonalisation problem of $T_{j}$ does not arise. For multi-dimensional $|\Phi\rangle$ to find $\Lambda_{j}$ one has to rely on special diagonalisation techniques such as, for instance, the nested Bethe ansatz. Below we consider only the simplest one-dimensional examples.

Lieb-Liniger model. The two-body S-matrix for the Lieb-Liniger model with vector $|\Phi\rangle$ in the irrep $\lambda$ of $\mathfrak{S}_{N}$ is $S_{i j}=B \mathbb{1}+A \pi_{\lambda}\left(\alpha_{i j}\right)$, where $A$ and $B$ are the reflection and and transmission coefficients (4.50). There are two representations for which $S$ is scalar: the anti-symmetric representation $\lambda=\left[1^{N}\right]$ corresponding to fermions and symmetric representation $\lambda=[N]$ corresponding to bosons.

1) Case of fermions. For $\lambda=\left[1^{N}\right]$ one has $\pi_{\lambda}\left(\alpha_{i j}\right)=-1$ for any $\alpha_{i j}$, so that $S=B-A=1$ and $\Lambda_{j}=1$ for any $j$. As a result, equations (4.67) become the familiar quantisation condition $e^{i p_{j} L}=1$ for momenta of free fermions put on a circle of length $L$. To find the wave function, we notice that $Y_{j}=-1$ for this case. Therefore, from (4.31) we obtain the following equation

$$
\Phi\left(\alpha_{j} \tau\right)=Y_{j} \Phi(\tau)=-\Phi(\tau)
$$

which is solved as $\Phi(\tau)=\operatorname{sign}(\tau) \Phi(e)$. Since $\Phi(e)$ belongs to the anti-symmetric irrep $\lambda=\left[1^{N}\right]$, we have $\Phi(e)=\mathscr{A}(\sigma \mid e)=\operatorname{sign}\left(\sigma^{-1}\right) \mathscr{A}(e \mid e)$. Up to an overall normalisation factor $\mathscr{A}(e \mid e)$, the Bethe wave function (4.27) is then given by the Slater determinant

$$
\Psi_{\left\{p_{j}\right\}}\left(q_{1}, \ldots, q_{N}\right)=\sum_{\tau \in \mathfrak{S}_{N}} \operatorname{sign}\left(\sigma^{-1} \tau\right) e^{i q_{\sigma(i)} p_{\tau(i)}}=\operatorname{det}\left(e^{i q_{i} p_{j}}\right) .
$$

2) Bose gas. For $\lambda=[N]$ one has $\pi_{\lambda}\left(\alpha_{i j}\right)=1$ for any $\alpha_{i j}$, so that $S=A+B$ which explicitly is

$$
S\left(p_{1}, p_{2}\right)=\frac{p_{1}-p_{2}-i \kappa}{p_{1}-p_{2}+i \kappa}, \quad \kappa>0 .
$$

The Bethe equation (4.67) reduce to

$$
e^{i p_{j} L}=\prod_{k \neq j}^{N} S\left(p_{k}, p_{j}\right)=\prod_{k \neq j}^{N} \frac{p_{j}-p_{k}+i \kappa}{p_{j}-p_{k}-i \kappa} .
$$

This set of $N$ equations determine the allowed values of the particle momenta in this model.The vector $\Phi$ in (4.62) is one-dimensional, i.e. the amplitude $\mathscr{A}(\sigma \mid \tau)$ does not depend on $\sigma$. From (4.31) we then have

$$
\mathscr{A}\left(\alpha_{j} \tau\right)=\frac{p_{\tau(j)}-p_{\tau(j+1)}-i \kappa}{p_{\tau(j)}-p_{\tau(j+1)}+i \kappa} \mathscr{A}(\tau) .
$$


The last equation has a unique, up to an overall normalisation, which we pick up to produce a solution

$$
\mathscr{A}(\tau)=\operatorname{sign} \tau \prod_{i<j}\left(p_{\tau(i)}-p_{\tau(j)}+i \kappa\right)
$$

Substituting this expression into the Bethe wave function (4.29), we find

$$
\begin{aligned}
\Psi\left(q_{1}, \ldots, q_{N}\right) & =\sum_{\sigma \in \mathfrak{S}_{N}} \sum_{\tau \in \mathfrak{S}_{N}} e^{i \sum_{k=1}^{N} q_{\sigma(k)} p_{\tau(k)}} \\
& \times \operatorname{sign} \tau \prod_{i<j}\left(p_{\tau(i)}-p_{\tau(j)}+i \kappa\right) \prod_{i=1}^{N-1} \Theta\left(q_{\sigma(i+1)}-q_{\sigma(i)}\right) .
\end{aligned}
$$

Using the invariance of the scalar product and making the shift $\tau \rightarrow \sigma \tau$, after which the sum over $\sigma$ can be performed with the following result

$$
\Psi_{\left\{p_{j}\right\}}\left(q_{1}, \ldots, q_{N}\right)=\prod_{i<j}^{N}\left(p_{i}-p_{j}\right) \sum_{\tau \in \mathfrak{S}_{N}} e^{i \sum_{k=1}^{N} q_{k} p_{\tau(k)}} \prod_{i<j}^{N}\left[1-\frac{i \kappa \epsilon\left(q_{i}-q_{j}\right)}{p_{\tau(i)}-p_{\tau(j)}}\right] .
$$

The wave function is parametrised by a set of $N$ momenta $\left\{p_{j}\right\}$ which was reflected in its notation above. The function is symmetric under permutations of coordinates and antisymmetric under permutations of momenta, so that it vanishes if any two momenta coincide. This result for the Bose gas is exact [22].

Rational CMS model. Second, we consider the rational CMS model for which the Bethe-Yang equations in the repulsive regime can be solved exactly. For this model the phase shift $\theta$ is given by $\theta(k)=\pi(\gamma-\hbar) \operatorname{sign}(k)$. The S-matrix reduces to the reflection amplitude $S=A \cdot \pi_{\lambda}=-e^{-i \theta} \cdot \pi_{\lambda}$, where $\pi_{\lambda}=1$ for bosons in the symmetric, $\lambda=[N]$, representation and $\pi_{\lambda}=-1$ for fermions in the anti-symmetric, $\lambda=\left[1^{N}\right]$, representation. Accordingly, the Bethe equations (4.67) are

$$
e^{\frac{i}{\hbar} p_{j} L}=(-1)^{(N-1) \sigma} \prod_{k \neq j}^{N} e^{i \pi(\beta-1) \operatorname{sign}\left(p_{j}-p_{k}\right)}, \quad \beta=\frac{\gamma}{\hbar},
$$

where $\sigma=0$ for fermions and $\sigma=1$ for bosons and we restored the the Planck constant $\hbar$. Equation (4.70) can be solved by passing to its logarithmic version

$$
p_{j}=\frac{2 \pi \hbar I_{j}}{L}+\frac{\pi \hbar(\beta-1)}{L} \sum_{k \neq j}^{N} \operatorname{sign}\left(p_{j}-p_{k}\right), \quad j=1, \ldots, N,
$$

where the numbers $I_{j}=n_{j}+\frac{N-1}{2} \sigma, n_{j} \in \mathbb{Z}$, arise from the phase ambiguity. They are integers for fermions for bosons with $N$ odd, and half-odd integers for bosons with $N$ even. It is convenient to order momenta as

$$
p_{1}<\ldots<p_{N}
$$


According to this ordering, $p_{k}<p_{j}$ for $k=1, \ldots, j-1$, and $p_{k}>p_{j}$ for $k=j+1, \ldots, N$. Then, summing up in (4.71), we find

$$
p_{j}=\frac{2 \pi \hbar I_{j}}{L}+\frac{2 \pi \hbar(\beta-1)}{L}\left(j-\frac{N+1}{2}\right)
$$

The distinguished case $\beta=1$ corresponds to free fermions or hard-core bosons. For this case the particle momenta are quantised as $p_{j}=2 \pi \hbar I_{j} / L$, where $I_{j}$ are distinct (half)-integers satisfying $I_{1}<\ldots<I_{N}$. Introducing a reduced length parameter $\ell=L / 2 \pi$, we can write the formula (4.73) as

$$
p_{j}=\frac{\hbar}{\ell}\left(I_{j}+\frac{N+1}{2}-j+\beta\left(j-\frac{N+1}{2}\right)\right) .
$$

A crucial observation is that this formula coincides with the expression for the quasi-momenta (3.21) of the trigonometric CMS model, provided we make the following identification of quantum numbers $I_{j}$ with integers $\lambda_{k}$ parametrising Young diagrams

$$
\lambda_{N+1-j}=I_{j}+\frac{N+1}{2}-j \text {. }
$$

The ground state of the trigonometric model has all $\lambda$ vanishing, which gives $I_{j}=j-\frac{N+1}{2}$ and $p_{j}=\frac{\gamma}{\ell}\left(j-\frac{N+1}{2}\right), N$ odd, for the ground state. The expressions for the total energy and momentum written in terms of $p_{j}$ are the same for both models, see (3.22). In the trigonometric model $p_{j}$ is a quasi-momentum and in the rational model it is an asymptotic momentum. Thus, solutions of the Bethe Ansatz equations for the rational CMS model yield the exact spectrum of its trigonometric (finite-size) counterpart. It is worth pointing out that while for the trigonometric model the length parameter $L=2 \pi \ell$ (the period) occurs directly in the hamiltonian, for the rational model it enters the spectrum only through the Bethe equations that follow from the periodicity condition imposed on the wave function.

\section{Two-dimensional integrable PDEs}

So far we dealt with systems with a finite number of degrees of freedom. In the rest of these lectures we will take a brief look at some interesting examples of infinite-dimensional hamiltonian systems which appear to be integrable.

Remarkably, there exist certain partial differential equations for functions depending on two variables $(x, t)$, which can be treated as integrable hamiltonian systems with infinite number of degrees of freedom. Examples include

- The Korteweg-de-Vries (KdV) equation

$$
\frac{\partial u}{\partial t}=6 u u_{x}-u_{x x x} .
$$

- The non-linear Schrodinger equation

$$
i \frac{\partial \psi}{\partial t}=-\psi_{x x}+2 \kappa|\psi|^{2} \psi
$$

where $\psi=\psi(x, t)$ is a complex-valued function. 
- The Sine-Gordon (SG) equation

$$
\frac{\partial^{2} \phi}{\partial t^{2}}-\frac{\partial^{2} \phi}{\partial x^{2}}+\frac{m^{2}}{\beta} \sin \beta \phi=0
$$

- The classical Heisenberg magnet

$$
\frac{\partial \vec{S}}{\partial t}=\vec{S} \times \frac{\partial^{2} \vec{S}}{\partial x^{2}}
$$

where $\vec{S}(x, t)$ lies on the unit sphere in $\mathbb{R}^{3}$.

The complete specification of each model requires also boundary and initial conditions. Among the important cases are

1) Rapidly decreasing case. We impose the condition that

$$
\psi(x, t) \rightarrow 0 \text { for }|x| \rightarrow \infty
$$

sufficiently fast, for instance, $\psi(x, t)$ belongs to the Schwarz space $\mathscr{L}\left(\mathbb{R}^{1}\right)$, that is $\psi$ is a differentiable function which vanishes faster than any power of $|x|^{-1}$ for $|x| \rightarrow \infty$.

2) Periodic boundary conditions. Here we require that $\psi$ is differentiable and satisfies the periodicity requirement

$$
\psi(x+2 \pi, t)=\psi(x, t)
$$

A characteristic feature of the above equations that they have solutions of a special type, the so-called solitons. The soliton was first discovered by accident by the naval architect, John Scott Russell, in August 1834 on the Glasgow to Edinburg channel. The modern theory originates from the work of Kruskal and Zabusky in 1965 [23].

\subsection{Soliton solutions}

Here we discuss the simplest cnoidal wave type (periodic) and also one-soliton solutions of the $\mathrm{KdV}$ and SG equations.

Korteweg-de-Vries cnoidal wave and soliton. By rescaling of $t, x$ and $u$ one can bring the KdV equation to the canonical form

$$
u_{t}+6 u u_{x}+u_{x x x}=0 .
$$

We will look for a solution of this equation in the form of a single-phase periodic wave of a permanent shape

$$
u(x, t)=u(x-v t),
$$

where $v=$ const is the phase velocity. Plugging this ansatz into the equation we obtain

$$
-v u_{x}+6 u u_{x}+u_{x x x}=\frac{d}{d x}\left(-v u+3 u^{2}+u_{x x}\right)=0 .
$$

We thus get

$$
-v u+3 u^{2}+u_{x x}+e=0
$$


where $e$ is an integration constant. Multiplying this equation with an integrating factor $u_{x}$ we get

$$
-v u u_{x}+3 u^{2} u_{x}+u_{x} u_{x x}+e u_{x}=\frac{d}{d x}\left(-\frac{v}{2} u^{2}+u^{3}+\frac{1}{2} u_{x}^{2}+e u\right)=0,
$$

We thus obtain

$$
u_{x}^{2}=k-2 e u+v u^{2}-2 u^{3}=-2\left(u-b_{1}\right)\left(u-b_{2}\right)\left(u-b_{3}\right),
$$

where $k$ is another integration constant. In the last equation we traded the integration constants $e, k$ for three parameters $b_{3} \geq b_{2} \geq b_{1}$ which satisfy the relation

$$
v=2\left(b_{1}+b_{2}+b_{3}\right) \text {. }
$$

Equation

$$
u_{x}^{2}=-2\left(u-b_{1}\right)\left(u-b_{2}\right)\left(u-b_{3}\right),
$$

describes motion of a "particle" with the coordinate $u$ and the time $x$ in the potential $V=2(u-$ $\left.b_{1}\right)\left(u-b_{2}\right)\left(u-b_{3}\right)$. Since $u_{x}^{2} \geq 0$ for $b_{2} \leq u \leq b_{3}$ the particle oscillates between the end points $b_{2}$ and $b_{3}$ with the period

$$
\ell=2 \int_{b_{2}}^{b_{3}} \frac{\mathrm{d} u}{\sqrt{-2\left(u-b_{1}\right)\left(u-b_{2}\right)\left(u-b_{3}\right)}}=\frac{2 \sqrt{2}}{\left(b_{3}-b_{2}\right)^{1 / 2}} \mathrm{~K}(m),
$$

where $\mathrm{K}(m)$ is an elliptic integral of the first kind and $m$ is an elliptic modulus $0 \leq m=\frac{b_{3}-b_{2}}{b_{3}-b_{1}} \leq 1$. The equation (5.1) can be integrated in terms of Jacobi elliptic cosine function $\mathrm{cn}(x, m)$ to give

$$
u(x, t)=b_{2}+\left(b_{3}-b_{2}\right) \mathrm{cn}^{2}\left(\sqrt{\left(b_{3}-b_{1}\right) / 2}\left(x-v t-x_{0}\right), m\right),
$$

where $x_{0}$ is an initial phase. This solution is often called as cnoidal wave. When $m \rightarrow 1$, i.e. $b_{2} \rightarrow b_{1}$, the cnoidal wave turns into a solitary wave

$$
u(x, t)=b_{1}+\frac{A}{\cosh ^{2}\left(\sqrt{\frac{A}{2}}\left(x-v t-x_{0}\right)\right)} .
$$

Here the velocity $v=2\left(b_{1}+b_{2}+b_{3}\right)=2\left(2 b_{1}+b_{3}\right)=2\left(3 b_{1}+b_{3}-b_{1}\right)$ is connected to the amplitude $A=b_{3}-b_{1}$ by the relation

$$
v=6 b_{1}+2 A .
$$

Here $u(x, t)=b_{1}$ is called a background flow because $u(x, t) \rightarrow b_{1}$ as $x \rightarrow \pm \infty$. One can further note that the background flow can be eliminated by a passage to a moving frame and using the invariance of the $\mathrm{KdV}$ equation with respect to the Galilean transformations $u \rightarrow u+d, x \rightarrow x-6 d t$, where $d$ is constant.

To sum up, the cnoidal waves form a three-parameter family of the KdV solutions while solitons are parametrised by two independent parameters (with an account of the background flow).

$\mathrm{KdV}$ and isospectral deformations. Consider the Sturm-Liouville operator

$$
L=\partial_{x}^{2}-u(x),
$$


where $x \in \mathbb{R}$. Assume that $u(x)$ develops in time according to the $\mathrm{KdV}$ equation $\left(u^{\prime} \equiv u_{x}\right)$

$$
\dot{u}-6 u u^{\prime}+u^{\prime \prime \prime}=0 \text {. }
$$

Then the spectrum of $L$ appears however to be time-independent under the KdV evolution. Below we prove this statement.

The spectral problem reads

$$
L \psi=\lambda \psi
$$

that is

$$
\psi^{\prime \prime}=(u+\lambda) \psi,
$$

Take the time derivative of this equation, we get

$$
\dot{\psi}^{\prime \prime}-\dot{u} \psi-(u+\lambda) \dot{\psi}=\dot{\lambda} \psi .
$$

Now multiply both sides by $\psi$

$$
\dot{\psi}^{\prime \prime} \psi-\dot{u} \psi^{2}-\underbrace{(u+\lambda) \psi}_{\psi^{\prime \prime}} \dot{\psi}=\dot{\lambda} \psi^{2} .
$$

Hence,

$$
\dot{\psi}^{\prime \prime} \psi-\psi^{\prime \prime} \dot{\psi}-\dot{u} \psi^{2}=\dot{\lambda} \psi^{2} .
$$

The fist two terms can be written as a total derivative, so we obtain

$$
\left(\dot{\psi}^{\prime} \psi-\dot{\psi} \psi^{\prime}\right)^{\prime}-\dot{u} \psi^{2}=\dot{\lambda} \psi^{2}
$$

Now we replace $\dot{u}$ for the right hand side of the $\mathrm{KdV}$ equation which gives

$$
\left(\dot{\psi}^{\prime} \psi-\dot{\psi} \psi^{\prime}\right)^{\prime}-\left(6 u u^{\prime}-u^{\prime \prime \prime}\right) \psi^{2}=\dot{\lambda} \psi^{2}
$$

Successively differentialting the spectral equation (5.3), we obtain

$$
\begin{aligned}
\psi^{\prime \prime \prime} & =(u+\lambda)^{\prime} \psi+(u+\lambda) \psi^{\prime} \\
\psi^{\prime \prime \prime \prime} & =(u+\lambda)^{\prime \prime} \psi+2(u+\lambda)^{\prime} \psi^{\prime}+(u+\lambda) \psi^{\prime \prime}, \\
\psi^{\prime \prime \prime \prime \prime} & =(u+\lambda)^{\prime \prime \prime} \psi+3(u+\lambda)^{\prime \prime} \psi^{\prime}+3(u+\lambda)^{\prime} \psi^{\prime \prime}+(u+\lambda) \psi^{\prime \prime \prime} .
\end{aligned}
$$

In the expression $u^{\prime \prime \prime} \psi^{2}=(u+\lambda)^{\prime \prime \prime} \psi^{2}$ we replace $(u+\lambda)^{\prime \prime \prime} \psi$ by using the last relation:

$$
\begin{aligned}
u^{\prime \prime \prime} \psi^{2} & =\psi^{\prime \prime \prime \prime \prime} \psi-3(u+\lambda)^{\prime \prime} \psi^{\prime} \psi-3(u+\lambda)^{\prime} \psi^{\prime \prime} \psi-(u+\lambda) \psi \psi^{\prime \prime \prime}= \\
& =\psi^{\prime \prime \prime \prime \prime} \psi-\psi^{\prime \prime} \psi^{\prime \prime \prime}-3(u+\lambda)^{\prime \prime} \psi^{\prime} \psi-3(u+\lambda)^{\prime} \psi^{\prime \prime} \psi= \\
& =\left(\psi^{\prime \prime \prime \prime} \psi-\psi^{\prime \prime \prime} \psi^{\prime}\right)^{\prime}-3(u+\lambda)^{\prime \prime} \psi^{\prime} \psi-3(u+\lambda)^{\prime} \psi^{\prime \prime} \psi .
\end{aligned}
$$

Analogously,

$$
u u^{\prime} \psi^{2}=u \psi\left(u^{\prime} \psi\right)=u \psi\left(\psi^{\prime \prime \prime}-(u+\lambda) \psi^{\prime}\right)=u\left(\psi^{\prime \prime \prime} \psi-(u+\lambda) \psi \psi^{\prime}\right)=
$$




$$
=u\left(\psi^{\prime \prime \prime} \psi-\psi^{\prime \prime} \psi^{\prime}\right)=u\left(\psi^{\prime \prime} \psi-\psi^{\prime} \psi^{\prime}\right)^{\prime}=(u+\lambda)\left(\psi^{\prime \prime} \psi-\psi^{\prime} \psi^{\prime}\right)^{\prime}-\lambda\left(\psi^{\prime \prime} \psi-\psi^{\prime} \psi^{\prime}\right)^{\prime} .
$$

On the other hand,

$$
(u+\lambda)\left(\psi^{\prime \prime} \psi-\psi^{\prime} \psi^{\prime}\right)^{\prime}=(u+\lambda)(u+\lambda)^{\prime} \psi^{2}=(u+\lambda)^{\prime} \psi^{\prime \prime} \psi .
$$

Thus, we can write

$$
u u^{\prime} \psi^{2}=\frac{1}{2}\left[(u+\lambda)\left(\psi^{\prime \prime} \psi-\psi^{\prime} \psi^{\prime}\right)^{\prime}+(u+\lambda)^{\prime} \psi^{\prime \prime} \psi\right]-\lambda\left(\psi^{\prime \prime} \psi-\psi^{\prime} \psi^{\prime}\right)^{\prime} .
$$

Finally, combining everything together, we find

$$
\begin{aligned}
& {\left[\dot{\psi}^{\prime} \psi-\dot{\psi} \psi^{\prime}+\psi^{\prime \prime \prime \prime} \psi-\psi^{\prime \prime \prime} \psi^{\prime}+6 \lambda\left(\psi^{\prime \prime} \psi-\psi^{\prime} \psi^{\prime}\right)\right]^{\prime}-} \\
& \quad-3(u+\lambda)^{\prime \prime} \psi^{\prime} \psi-3(u+\lambda)^{\prime} \psi^{\prime \prime} \psi-\frac{6}{2}\left[(u+\lambda)\left(\psi^{\prime \prime} \psi-\psi^{\prime} \psi^{\prime}\right)^{\prime}+(u+\lambda)^{\prime} \psi^{\prime \prime} \psi\right]=\dot{\lambda} \psi^{2},
\end{aligned}
$$

which upon simplification gives

$$
\begin{aligned}
& {\left[\dot{\psi}^{\prime} \psi-\dot{\psi} \psi^{\prime}+\psi^{\prime \prime \prime \prime} \psi-\psi^{\prime \prime \prime} \psi^{\prime}+6 \lambda\left(\psi^{\prime \prime} \psi-\psi^{\prime} \psi^{\prime}\right)\right]^{\prime}-} \\
& \quad-3(u+\lambda)^{\prime \prime} \psi^{\prime} \psi-6(u+\lambda)^{\prime} \psi^{\prime \prime} \psi-3(u+\lambda)\left(\psi^{\prime \prime} \psi-\psi^{\prime} \psi^{\prime}\right)^{\prime}=\dot{\lambda} \psi^{2},
\end{aligned}
$$

One can further note that

$$
\begin{aligned}
(u+\lambda)^{\prime \prime} \psi^{\prime} \psi+2(u+\lambda)^{\prime} \psi^{\prime \prime} \psi+(u+\lambda)\left(\psi^{\prime \prime} \psi-\psi^{\prime} \psi^{\prime}\right)^{\prime}= \\
=\left[(u+\lambda) \psi^{\prime \prime} \psi+(u+\lambda)^{\prime} \psi^{\prime} \psi-(u+\lambda) \psi^{\prime} \psi^{\prime}\right]^{\prime}= \\
=\left[\left((u+\lambda) \psi^{\prime}\right)^{\prime} \psi-\left((u+\lambda) \psi^{\prime}\right) \psi^{\prime}\right]^{\prime}
\end{aligned}
$$

With this relation at hand, we obtain

$$
\dot{\lambda} \psi^{2}=\left[\left(\dot{\psi}+\psi^{\prime \prime \prime}+6 \lambda \psi^{\prime}-3(u+\lambda) \psi^{\prime}\right)^{\prime} \psi-\left(\dot{\psi}+\psi^{\prime \prime \prime}+6 \lambda \psi^{\prime}-3(u+\lambda) \psi^{\prime}\right) \psi^{\prime}\right]^{\prime}
$$

Hence, introducing the short-hand notation

$$
\mathscr{W}=\dot{\psi}+\psi^{\prime \prime \prime}-3(u-\lambda) \psi^{\prime},
$$

we will get

$$
\dot{\lambda} \psi^{2}=\left[\mathscr{W}^{\prime} \psi-\mathscr{W} \psi^{\prime}\right]^{\prime}
$$

The right hand side is the total derivative. Thus, if the field $u$ decreases sufficiently fast at infinity, we find that

$$
\dot{\lambda} \int \psi^{2} \mathrm{~d} x=0
$$

which implies that $\dot{\lambda}=0$, that is an eigenvalue $\lambda$ does not depend on time. The $\mathrm{KdV}$ deformation of the Sturm-Liouville operator is isospectral. From the point of view of dynamical systems this means that the spectrum of the Sturm-Liouville operator comprises integrals of motion of the KdV equation. 
Sine-Gordon cnoidal wave and soliton. Consider the Sine-Gordon equation

$$
\phi_{t t}-\phi_{x x}+\frac{m^{2}}{\beta} \sin \beta \phi=0,
$$

where we assume that the functions $\phi(x, t)$ and $\phi(x, t)+2 \pi / \beta$ are assumed to be equivalent. Make an ansatz

$$
\phi(x, t)=\phi(x-v t)
$$

which leads to

$$
\left(v^{2}-1\right) \phi_{x x}+\frac{m^{2}}{\beta} \sin \beta \phi=0 .
$$

This can be integrated once

$$
C=\frac{v^{2}-1}{2} \phi_{x}^{2}-\frac{m^{2}}{\beta^{2}} \cos \beta \phi=\frac{v^{2}-1}{2} \phi_{x}^{2}+\frac{2 m^{2}}{\beta^{2}} \sin ^{2} \frac{\beta \phi}{2}-\frac{m^{2}}{\beta^{2}},
$$

where $C$ is an integration constant. This is nothing else but the conservation law of energy for the mathematical pendulum in the gravitational field of the Earth. We further bring the equation to the form

$$
\phi_{x}^{2}=\frac{2}{v^{2}-1}\left(C+\frac{m^{2}}{\beta^{2}}-\frac{2 m^{2}}{\beta^{2}} \sin ^{2} \frac{\beta \phi}{2}\right) .
$$

After making a substitution $y=\sin \frac{\beta \phi}{2}$ the equation becomes

$$
\left(y^{\prime}\right)^{2}=\frac{m^{2}}{\left(v^{2}-1\right)}\left(1-y^{2}\right)\left(\frac{C+\frac{m^{2}}{\beta^{2}}}{\frac{2 m^{2}}{\beta^{2}}}-y^{2}\right) .
$$

This equation has solutions in terms of elliptic functions which are analogous to the cnoidal waves of the KdV equation. However, as is known, the pendulum has three phases: oscillatory (an elliptic solution), rotatory (an elliptic solution) and motion with an infinite period. The later solution is precisely the one that would correspond to the SG soliton we are interested in. Assuming $v^{2}<1$, we see ${ }^{18}$ that such a solution would arise from (5.4) if we take $C=-\frac{m^{2}}{\beta^{2}}$. In this case equation (5.4) reduces to

$$
\phi_{x}=\frac{2 m}{\beta \sqrt{1-v^{2}}} \sin \frac{\beta \phi}{2} .
$$

This can be integrated to ${ }^{19}$

$$
\phi(x, t)=\sigma \frac{4}{\beta} \arctan \exp \left(\frac{m\left(x-v t-x_{0}\right)}{\sqrt{1-v^{2}}}\right) .
$$

Here $\sigma= \pm 1$. This solution can be interpreted in terms of relativistic particle moving with the velocity $v$. The field $\phi(x, t)$ has an important characteristic - topological charge

$$
Q=\frac{\beta}{2 \pi} \int \mathrm{d} x \frac{\partial \phi}{\partial x}=\frac{\beta}{2 \pi}(\phi(\infty)-\phi(-\infty)) .
$$

\footnotetext{
${ }^{18}$ Restoring the speed of light $c$ this condition for the velocity becomes $v^{2}<c^{2}$, i.e., the center of mass of the soliton cannot propagate faster than light.

${ }^{19}$ If $\phi(x, t)$ is a solution then $-\phi(x, t)$ is also a solution, as follows from (5.4).
} 
On the solutions one gets

$$
Q=\frac{\beta}{2 \pi}\left(\sigma \frac{4}{\beta}\right)\left(\frac{\pi}{2}-0\right)=\sigma .
$$

In addition to the continuous parameters $v$ and $x_{0}$, the soliton of the SG model has another important discrete characteristic - topological charge $Q=\sigma$. Solutions with $Q=1$ are called solitons (kinks), while solutions with $Q=-1$ are called ani-solitons (anti-kinks).

The stability of solitons finds its origin in the fine balance of nonlinearity and dispersion in the corresponding PDEs. Nonlinearity drives a solitary wave to concentrate further; dispersion is the effect to spread such a localized wave. If one of these two competing effects is lost, solitons become unstable and, eventually, disappear. In this respect, solitons are completely different from linear waves.

The SG model has even more sophisticated solutions. Consider the following function

$$
\phi(x, t)=\frac{4}{\beta} \arctan \frac{\omega_{2}}{\omega_{1}} \frac{\sin \left(\frac{m \omega_{1}(t-v x)}{\sqrt{1-v^{2}}}+\phi_{0}\right)}{\cosh \left(\frac{m \omega_{2}\left(x-v t-x_{0}\right)}{\sqrt{1-v^{2}}}\right)} .
$$

This is solution of the SG model called the double-soliton or breather. In addition to the uniform motion with velocity $v$ corresponding to a relativistic particle, the breather oscillates both in space and in time with frequencies $\frac{m v \omega_{1}}{\sqrt{1-v^{2}}}$ and $\frac{m \omega_{1}}{\sqrt{1-v^{2}}}$, respectively. The parameter $\phi_{0}$ plays the role of the initial phase. In particular, if $v=0$ the breather is a time-periodic solution of the SG equation. It has zero topological charge and can be interpreted as the bound state of the soliton and anti-soliton.

\subsection{Zero curvature representation}

The inverse scattering method (the method of solving a certain class of integrable non-linear PDEs) is based on the following remarkable observation. A two-dimensional PDE appears as the consistency condition of the overdetermined system of equations

$$
\begin{aligned}
& \frac{\partial \Psi}{\partial x}=U(x, t, \lambda) \Psi, \\
& \frac{\partial \Psi}{\partial t}=V(x, t, \lambda) \Psi .
\end{aligned}
$$

for a proper choice of the matrices $U(x, t, \lambda)$ and $V(x, t, \lambda)$. The consistency condition arises upon differentiation the first equation with respect to $t$ and the second with respect to $x$ :

$$
\begin{aligned}
& \frac{\partial^{2} \Psi}{\partial t \partial x}=\partial_{t} U(x, t, \lambda) \Psi+U(x, t, \lambda) \partial_{t} \Psi=\left(\partial_{t} U(x, t, \lambda)+U(x, t, \lambda) V(x, t, \lambda)\right) \Psi \\
& \frac{\partial^{2} \Psi}{\partial x \partial t}=\partial_{x} V(x, t, \lambda) \Psi+V(x, t, \lambda) \partial_{x} \Psi=\left(\partial_{x} V(x, t, \lambda)+V(x, t, \lambda) U(x, t, \lambda)\right) \Psi
\end{aligned}
$$

which implies the fulfilment of the following relation

$$
\partial_{t} U-\partial_{x} V+[U, V]=0 .
$$

This relation can be viewed as an analogue of the Lax representation (1.55). In the case when there is no $x$-dependence, (5.6) turns into (1.55) with the identification $L=U$ and $M=V$. 
If we introduce a gauge field $\mathscr{L}_{\alpha}$ with components $\mathscr{L}_{x}=U, \mathscr{L}_{t}=V$, then (5.6) can be interpreted as a condition of vanishing of the curvature $F_{\alpha \beta}$ of $\mathscr{L}$

$$
\partial_{\alpha} \mathscr{L}_{\beta}-\partial_{\beta} \mathscr{L}_{\alpha}-\left[\mathscr{L}_{\alpha}, \mathscr{L}_{\beta}\right]=0
$$

Correspondingly, we will refer to (5.6), (5.7) as the Lax representation or, equivalently, as the zero curvature condition (representation). Below we present two examples of differential equations admitting the zero curvature representation.

Example: KdV equation. Introduce the following $2 \times 2$ matrices

$$
U=\left(\begin{array}{cc}
0 & 1 \\
\lambda+u & 0
\end{array}\right), \quad V=\left(\begin{array}{cc}
u_{x} & 4 \lambda-2 u \\
4 \lambda^{2}+2 \lambda u+u_{x x}-2 u^{2} & -u_{x}
\end{array}\right) .
$$

On can verify that

$$
\partial_{t} U-\partial_{x} V+[U, V]=\left(\begin{array}{cc}
0 & 0 \\
u_{t}+6 u u_{x}-u_{x x x} & 0
\end{array}\right) .
$$

Note that there is yet another representation of the KdV equation which has the Lax form (1.55) and does not involve the spectral parameter. This is a representation by means of differential operators, namely,

$$
L=\partial_{x}^{2}-u, \quad M=-4 \partial_{x}^{3}+6 u \partial_{x}+3 u_{x} .
$$

One can verify that with this choice for $L$ and $M$ equation (1.55) reduces to the KdV equation (5.2). According to the general theory, the spectrum of $L$ must be conserved, which explains the isospectral property of the Sturm-Liouville operator that we have proved earlier by direct means.

Example: SG equation. Introduce the following $2 \times 2$ matrices

$$
\begin{aligned}
U & =\frac{\beta}{4 i} \phi_{t} \sigma_{3}+\frac{k_{0}}{i} \sin \frac{\beta \phi}{2} \sigma_{1}+\frac{k_{1}}{i} \cos \frac{\beta \phi}{2} \sigma_{2}, \\
V & =\frac{\beta}{4 i} \phi_{x} \sigma_{3}+\frac{k_{1}}{i} \sin \frac{\beta \phi}{2} \sigma_{1}+\frac{k_{0}}{i} \cos \frac{\beta \phi}{2} \sigma_{2},
\end{aligned}
$$

where $\sigma_{i}$ are the Pauli matrices and

$$
k_{0}=\frac{m}{4}\left(\lambda+\frac{1}{\lambda}\right), \quad k_{1}=\frac{m}{4}\left(\lambda-\frac{1}{\lambda}\right) .
$$

Again, by direct computation one verifies that the condition of zero curvature is equivalent to the SG equation.

Consider now a model which admits the zero curvature representation (5.6) for fields satisfying periodic boundary conditions with the period $2 \pi$. The one-parameter family of the flat connections allows one to define the monodromy matrix $\mathrm{T}(\lambda)$ which is the path-ordered exponential of the Lax component $U(\lambda)$ :

$$
\mathrm{T}(\lambda)=\mathscr{P} \exp \int_{0}^{2 \pi} \mathrm{d} x U(\lambda) .
$$

Let us derive the time evolution equation for this matrix. We have

$$
\partial_{t} \mathrm{~T}(\lambda)=\int_{0}^{2 \pi} \mathrm{d} x \mathscr{P}_{e}^{\int_{x}^{2 \pi} \mathrm{d} y U}\left(\partial_{t} U\right) \mathscr{P}_{e}^{\int_{0}^{x} \mathrm{~d} y U}
$$




$$
=\int_{0}^{2 \pi} \mathrm{d} x \mathscr{P} e^{\int_{x}^{2 \pi} \mathrm{d} y U}\left(\partial_{x} V+[V, U]\right) \mathscr{P} e^{\int_{0}^{x} \mathrm{~d} y U},
$$

where in the last formula we used the flatness of $\mathscr{L}_{\alpha} \equiv(U, V)$. The integrand of (5.9) is the total derivative

$$
\partial_{t} \mathrm{~T}(\lambda)=\int_{0}^{2 \pi} \mathrm{d} x \partial_{x}\left(\mathscr{P} e^{\int_{x}^{2 \pi} \mathrm{d} y U} V \mathscr{P} e^{\int_{0}^{x} \mathrm{~d} y U}\right)
$$

Thus, we obtained the following evolution equation

$$
\partial_{t} \mathrm{~T}(\lambda)=[V(2 \pi, t, \lambda), \mathrm{T}(\lambda)] .
$$

This formula shows that the eigenvalues of $\mathrm{T}(\lambda)$ generate an infinite set of integrals of motion upon expansion in $\lambda$. Thus, the spectral properties of the model are encoded into the monodromy matrix. In particular, one can define a Riemann surface $P(\lambda, \zeta)=\operatorname{det}(\mathrm{T}(\lambda)-\zeta \mathbb{1})=0$, where $\zeta, \lambda \in \mathbb{C}$, analogous to the spectral curve (1.69) in the finite-dimensional case. The coefficients of $P(\lambda, \zeta)$ are integrals of motion. The word "monodromy" for T comes from the fact that $\mathrm{T}(t)$ represents the monodromy of a solution of the fundamental linear system (5.5)

$$
\Psi(2 \pi, t)=\mathrm{T}(t) \Psi(0, t) .
$$

Indeed, if we differentiate this equation over $t$ we get

$$
\partial_{t} \Psi(2 \pi, t)=\partial_{t} \mathrm{~T} \Psi(0, t)+\mathrm{T} \partial_{t} \Psi(0, t),
$$

which, according to the fundamental linear system, gives

$$
\mathscr{L}_{t}(2 \pi, t) \mathrm{T} \Psi(0, t)=\partial_{t} \mathrm{~T} \Psi(0, t)+\mathrm{T} \mathscr{L}_{t}(0, t) \Psi(0, t) .
$$

This leads to the same equation for the time evolution of the monodromy matrix as found before

$$
\partial_{t} \mathrm{~T}=\left[\mathscr{L}_{t}, \mathrm{~T}\right]
$$

\subsection{Local integrals of motion}

The Lax representation of the two-dimensional PDE allows one to exhibit an infinite number of conservation laws. The procedure to derive the local conservation laws from the Lax representation is known as abelianization procedure and we will outline it below.

Starting from the zero-curvature condition (5.6), we assume that the matrices $U(x, t, \lambda)$ and $V(x, t, \lambda)$ depend on the spectral parameter $\lambda$ in a rational way and that they have poles at constant, i.e. $x, t$-independent, values of $\lambda=\lambda_{k}$. Thus, we can write

$$
\begin{aligned}
U & =U_{0}+\sum_{k} U_{k}, & U_{k} & =\sum_{r=-n_{k}}^{-1} U_{k, r}(x, t)\left(\lambda-\lambda_{r}\right)^{r}, \\
V & =V_{0}+\sum_{k} V_{k}, & V_{k} & =\sum_{r=-m_{k}}^{-1} V_{k, r}(x, t)\left(\lambda-\lambda_{r}\right)^{r} .
\end{aligned}
$$


Matching the pole structure in the zero curvature equations shows that that these equations are always compatible: there is one more variable than the number of equations, but there is a gauge transformation (see below) of the connection $(U, V)$ which leaves the zero-curvature condition invariant.

To understand solutions of the zero-curvature condition, we will perform a local analysis around a pole $\lambda=\lambda_{k}$. Our aim is to show that around each singularity one can perform a gauge transformation which brings the matrices $U(\lambda)$ and $V(\lambda)$ to a diagonal form. Finally, to make the consideration as simple as possible, we assume that the pole is located at zero.

In the neighbourhood of $\lambda=0$ the functions $U$ and $V$ can be expanded into Laurent series

$$
U(x, t, \lambda)=\sum_{r=-n}^{\infty} U_{r}(x, t) \lambda^{r}, \quad V(x, t, \lambda)=\sum_{r=-m}^{\infty} V_{r}(x, t) \lambda^{r} .
$$

Let $g \equiv g(x, t, \lambda)$ be a regular gauge transformation around $\lambda=0$ that is

$$
g=\sum_{r=0}^{\infty} g_{r} \lambda^{r}, \quad g^{-1}=\sum_{r=0}^{\infty} h_{r} \lambda^{r} .
$$

Consider the gauge transformation

$$
\begin{aligned}
& \widetilde{U}=g U g^{-1}+\partial_{x} g g^{-1} \\
& \widetilde{V}=g V g^{-1}+\partial_{t} g g^{-1}
\end{aligned}
$$

Consider the transition matrix $\mathrm{T}(x, y, \lambda)$ which is a solution of the differential equation

$$
\left(\partial_{x}-U(x, \lambda)\right) \mathrm{T}(x, y, \lambda)=0
$$

satisfying the initial condition $\mathrm{T}(x, x, \lambda)=\mathbb{1}$. Formally such a solution is given by the path-ordered exponent

$$
\mathrm{T}(x, y, \lambda)=\mathscr{P} e^{\int_{y}^{x} \mathrm{~d} z U(z, \lambda)} .
$$

Under the gauge transformation we have

$$
g(x, \lambda)\left(\partial_{x}-U(x, \lambda)\right) g^{-1}(x, \lambda) \mathrm{T}_{g}(x, y, \lambda)=0,
$$

where

$$
\mathrm{T}_{g}(x, y, \lambda)=g(x, \lambda) \mathrm{T}(x, y, \lambda) g^{-1}(y, \lambda) .
$$

is the transition matrix for the gauged-transformed connection $\widetilde{U}$, which also obeys the condition $\mathrm{T}_{g}(x, x, \lambda)=\mathbb{1}$. This formula shows how the transition matrix transforms under the gauge transformations of the Lax connection. By means of a regular gauge transformation the transition matrix can be diagonalized around every pole of the matrix $U$, namely,

$$
\mathrm{T}(x, y, \lambda)=g(x, \lambda) \exp (D(x, y, \lambda)) g^{-1}(y, \lambda),
$$

where

$$
D(x, y, \lambda)=\sum_{r=-n}^{\infty} D_{r}(x, y) \lambda^{r}
$$


is the diagonal matrix. Below we consider a concrete example which illustrates the abelianization procedure, as well as the technique of constructing local integrals of motion.

Example: The Heisenberg model. We start with the definition of the model classical Heisenberg model. Consider a spin variable $S(x)$ :

$$
S(x)=\sum_{i} S^{i}(x) \sigma_{i}
$$

Clearly, $S^{i}(x)^{2}=s^{2}$. Here $\sigma^{i}$ are the standard Pauli matrices obeying the relations

$$
\left[\sigma_{i}, \sigma_{j}\right]=2 i \epsilon_{i j k} \sigma_{k}, \quad \operatorname{Tr}\left(\sigma_{i} \sigma_{j}\right)=2 \delta_{i j} .
$$

The spins $S^{i}(x)$ are the dynamical variables subject to the Poisson structure

$$
\left\{S^{i}(x), S^{j}(y)\right\}=\epsilon^{i j k} S^{k}(x) \delta(x-y) .
$$

The phase space is thus infinite-dimensional. The hamiltonian of the model is

$$
H=-\frac{1}{4} \int_{0}^{2 \pi} \mathrm{d} x \operatorname{Tr}\left(\partial_{x} S \partial_{x} S\right)
$$

and the equations of motion are

$$
\partial_{t} S(x)=\{H, S(x)\}=-\frac{1}{4} \int_{0}^{2 \pi} \mathrm{d} y\left\{\operatorname{Tr}\left(\partial_{y} S \partial_{y} S\right), S(y)\right\}=\epsilon^{i j k} S^{i}(x) \partial_{x}^{2} S^{j}(x) \sigma_{k} .
$$

These equations can be compactly written as

$$
\partial_{t} S=-\frac{i}{2}\left[S, \partial_{x}^{2} S\right]=-\frac{i}{2} \partial_{x}\left[S, \partial_{x} S\right]
$$

These are the Landau-Lifshitz equations. Written in the form (5.12), they admit a generalisation to any Lie algebra. If we introduce the $\mathfrak{s u}(2)$-valued current $J$ with components

$$
J_{x}=S, \quad J_{t}=-\frac{i}{2}\left[S, \partial_{x} S\right],
$$

then the equations of motion take the form of the current conservation

$$
\partial_{t} J_{x}-\partial_{x} J_{t}=0
$$

which is $\epsilon^{\alpha \beta} \partial_{\alpha} J_{\beta}=0$.

Integrability of the Landau-Lifshitz equations relies on the fact that they can be obtained from the zero curvature condition

$$
\left(\partial_{\alpha}-\mathscr{L}_{\alpha}\right) \Psi(x, t)=0
$$

Here

$$
\begin{aligned}
\mathscr{L}_{x} & =-\frac{i}{\lambda} S(x), \\
\mathscr{L}_{t} & =-\frac{2 i s^{2}}{\lambda^{2}} S(x)-\frac{1}{2 \lambda}\left[S(x), \partial_{x} S(x)\right] .
\end{aligned}
$$


Indeed,

$$
\begin{aligned}
\partial_{t} \mathscr{L}_{x}-\partial_{x} \mathscr{L}_{t}+\left[\mathscr{L}_{x}, \mathscr{L}_{t}\right] & =-\frac{i}{\lambda} \partial_{t} S(x)+\frac{2 i s^{2}}{\lambda^{2}} \partial_{x} S(x) \\
& +\frac{1}{2 \lambda} \partial_{x}\left[S(x), \partial_{x} S(x)\right]+\frac{i}{2 \lambda^{2}}\left[S(x),\left[S(x), \partial_{x} S(x)\right]\right]=0 .
\end{aligned}
$$

Further, computing the Poisson bracket between the components $\mathscr{L}_{x} \equiv U(x, \lambda)$ of the Lax connection, we find

$$
\{U(x, \lambda), U(y, \mu)\}=-\frac{1}{\lambda \mu}\left\{S^{i}(x), S^{j}(y)\right\} \sigma_{i} \otimes \sigma_{j}=-\frac{1}{\lambda \mu} \epsilon^{i j k} S^{k}(x) \sigma_{i} \otimes \sigma_{j} \delta(x-y) .
$$

On the other hand,

$$
\begin{aligned}
& {\left[\frac{\sigma_{i} \otimes \sigma_{i}}{\lambda-\mu}, U(x, \lambda) \otimes \mathbb{I}+\mathbb{I} \otimes U(y, \mu)\right] \delta(x-y)=-\left[\frac{\sigma_{i} \otimes \sigma_{i}}{\lambda-\mu}, \frac{i}{\lambda} S(x) \otimes \mathbb{I}+\mathbb{I} \otimes \frac{i}{\mu} S(y)\right] \delta(x-y)} \\
& =-\frac{i}{\lambda-\mu} S^{k}(x)\left(\frac{1}{\lambda}\left[\sigma_{i}, \sigma_{k}\right] \otimes \sigma_{i}+\frac{1}{\mu} \sigma_{i} \otimes\left[\sigma_{i}, \sigma_{k}\right]\right) \delta(x-y)= \\
& \quad=\frac{2}{\lambda-\mu}\left(\frac{1}{\lambda}-\frac{1}{\mu}\right) \epsilon^{i j k} S^{k}(x) \sigma_{i} \otimes \sigma_{j} \delta(x-y)=-\frac{2}{\lambda \mu} \epsilon^{i j k} S^{k}(x) \sigma_{i} \otimes \sigma_{j} \delta(x-y) .
\end{aligned}
$$

Thus, we observe that the Poisson bracket between the components of the Lax connection admits in the $r$-matrix form

$$
\{U(x, \lambda), U(y, \mu)\}=[r(\lambda, \mu), U(x, \lambda) \otimes \mathbb{I}+\mathbb{I} \otimes U(y, \mu)] \delta(x-y),
$$

where the classical spectral-dependent $r$-matrix appears to be

$$
r(\lambda, \mu)=\frac{1}{2} \frac{\sigma_{i} \otimes \sigma_{i}}{\lambda-\mu} .
$$

This form of the brackets between the components of the Lax connection implies that the Poisson bracket between the components of the monodromy matrix

$$
\mathrm{T}(\lambda)=\mathscr{P} \exp \left[\int_{0}^{2 \pi} \mathrm{d} x U(x, \lambda)\right]
$$

is

$$
\{\mathrm{T}(\lambda) \otimes \mathrm{T}(\mu)\}=[r(\lambda, \mu), \mathrm{T}(\lambda) \otimes \mathrm{T}(\mu)] .
$$

This is the quadratic Sklyanin bracket. It is quadratic in the matrix elements of the monodromy matrix.

From the definition, $\mathrm{T}(\lambda)$ is analytic (entire) in $\lambda$ with an essential singularity at $\lambda=0$. It is straigntforward to find the expansion around $\lambda=\infty$ :

$$
\mathrm{T}(\lambda)=\mathbb{I}+\frac{i}{\lambda} \int_{0}^{2 \pi} \mathrm{d} x S(x)-\frac{1}{\lambda^{2}} \int_{0}^{2 \pi} \mathrm{d} x S(x) \int_{0}^{x} \mathrm{~d} y S(y)+\cdots
$$

The development in $1 / \lambda$ has an infinite radius of convergency. 
To find the structure of $\mathrm{T}(\lambda)$ around $\lambda=0$ is more delicate but equally important as this yields the local conserved charges in involution. To proceed, introduce the so-called partial monodromy

$$
\mathrm{T}(x, \lambda)=\mathscr{P} \exp \left[\int_{0}^{x} \mathrm{~d} y U(y, \lambda)\right] .
$$

The main point is to note that there exists a local gauge transformation, regular at $\lambda=0$, such that

$$
\mathrm{T}(x, \lambda)=g(x) D(x) g^{-1}(0),
$$

where $D(x)=\exp \left(i d(x) \sigma_{3}\right)$ is a diagonal matrix. We can choose $g$ to be unitary, and, since $g$ is defined up to to a diagonal matrix, we can require that it has a real diagonal part

$$
g=\frac{1}{(1+v \bar{v})^{\frac{1}{2}}}\left(\begin{array}{cc}
1 & v \\
-\bar{v} & 1
\end{array}\right) .
$$

Then the differenial equation for the monodromy

$$
\partial_{x} \mathrm{~T}=U \mathrm{~T}=-\frac{i}{\lambda} S \mathrm{~T}
$$

becomes a differential equation for $g$ and $d$ :

$$
g^{-1} \partial_{x} g+i \partial_{x} d \sigma_{3}+\frac{i}{\lambda} g^{-1} S g=0 .
$$

We project this equation on the Pauli matrices and get

$$
\begin{aligned}
& \partial_{x} v=-\frac{i}{\lambda}\left(S_{-}+2 v S_{3}-S_{+} v^{2}\right) \\
& \partial_{x} d=\frac{1}{2 \lambda}\left(-2 S_{3}+v S_{+}+\bar{v} S_{-}\right) .
\end{aligned}
$$

The first of these equations is a Riccati equation for $v(x)$. Expanding in $\lambda$ the functions $v(x)$ and $d(x)$ as

$$
\begin{aligned}
& \partial_{x} d=-\frac{s}{\lambda}+\sum_{n=0}^{\infty} \rho_{n}(x) \lambda^{n} \\
& v(x)=\sum_{n=0}^{\infty} v_{n}(x) \lambda^{n}, \quad v_{0}=\frac{S_{3}-s}{S_{+}},
\end{aligned}
$$

we rewrite the Riccati equation in the form

$$
2 i s v_{n+1}=-v_{n}^{\prime}+i S_{+} \sum_{m=1}^{n} v_{n+1-m} v_{m}
$$

and

$$
\rho_{n}=\frac{1}{2}\left(v_{n+1} S_{+}+\bar{v}_{n+1} S_{-}\right) .
$$

Note that $v(x)$ is regular at $\lambda=0$. Equations above recursively determine the functions $v_{n}(x)$ and $\rho_{n}(x)$ as local functions of the dynamical variables $S^{i}(x)$. This describes the asymptotic behavior 
of $\mathrm{T}(\lambda)$ around $\lambda=0$. The asymptotic series become convergent if we regularize the model by discretizing the space interval.

Concerning the monodromy matrix $\mathrm{T}(\lambda)$, since $g(x)$ is local and if we assume periodic boundary conditions, we can write

$$
\mathrm{T}(\lambda)=\cos p(\lambda) \mathbb{I}+i \sin p(\lambda) M(\lambda)
$$

where $M(\lambda)=g(0) \sigma_{3} g(0)^{-1}$ and

$$
p(\lambda)=\int_{0}^{2 \pi} \mathrm{d} x \partial_{x} d
$$

The trace of the monodromy matrix, called the transfer matrix, is

$$
\operatorname{Tr} \operatorname{T}(\lambda)=2 \cos p(\lambda)
$$

Thus, $p(\lambda)$ is the generating function for the commuting local conserved quantities

$$
H_{n+1}=\int_{0}^{2 \pi} \mathrm{d} x \rho_{n}(x), \quad n=0,1, \ldots
$$

The first three integrals are

$$
\begin{aligned}
& H_{1}=\frac{i}{4 s} \int_{0}^{2 \pi} \mathrm{d} x \log \left(\frac{S_{+}}{S_{-}}\right) \partial_{x} S_{3}, \\
& H_{2}=-\frac{1}{16 s^{3}} \int_{0}^{2 \pi} \mathrm{d} x \operatorname{tr}\left(\partial_{x} S \partial_{x} S\right), \\
& H_{3}=\frac{i}{64 s^{5}} \int_{0}^{2 \pi} \mathrm{d} x \operatorname{tr}\left(S\left[\partial_{x} S, \partial_{x}^{2} S\right]\right) .
\end{aligned}
$$

The integrals $H_{1}$ and $H_{2}$ are the momentum and energy, respectively.

This discussion finishes our brief presentation of some properties of integrable PDEs. Concerning finding explicit solutions of such equations, the powerful inverse scattering method and algebro-geometric (finite-gap) integration were developed basing on the existence of Lax representation. The reader is invited to consult, e.g., [24, 25], [6] on thorough explanation of these techniques.

\section{Acknowledgments}

It is my great delight to thank Prof. Armen Nersessian for the kind invitation to deliver these lectures and the members of the Theory Department of the Yerevan Physics Institute for the warm hospitality and very inspiring discussions. I also want to thank the organisers of the RDP online workshop "Recent Advances in Mathematical Physics", 5-6 December 2020, for giving me the opportunity to present my current research that partially relies on the material presented in these lectures. 


\section{References}

[1] R. Abraham and J.E. Marsden, Foundations of Mechanics, AMS, 1978.

[2] S. Kobayashi and K. Nomizu, Foundations of the Differential Geometry, vol. 1, John Wiley \& Sons, Inc., 1996.

[3] V. Arnold, Mathematical methods of classical mechanics, vol. 60, Springer, 1989.

[4] M.A. Olshanetsky and A.M. Perelomov, Classical integrable finite dimensional systems related to Lie algebras, Phys. Rept. 71, 313, 1981.

[5] O. Babelon and C.M. Viallet, Hamiltonian Structures and Lax Equations, Phys. Lett. B237, 411-416, 1990.

[6] O. Babelon, D. Bernard and M. Talon, Introduction to Classical Integrable Systems, Cambridge University Press, 2003.

[7] A.A. Kirillov, Lectures on the Orbit Method, AMS, 2004.

[8] J. Marsden and A. Weinstein, Reduction of symplectic manifolds with symmetry, Rep. Math. Phys., vol. 5, 1974, 121-130.

[9] G. Arutyunov, Elements of classical and quantum integrable systems, Springer, 2019.

[10] D. Kazhdan, B. Kostant, and S. Sternberg, Hamiltonian group actions and dynamical systems of calogero type, Communications on Pure and Applied Mathematics 31(4), 481-507, 1978.

[11] M. Adler, P. van Moerbeke and P. Vanhaeche, Algebraic integrability, Painlevé Geometry and Lie Algebras, Springer Verlag, 2004.

[12] G. Arutyunov and S. Frolov, Quantum Dynamical R-matrices and Quantum Frobenius Group, Commun. Math. Phys. 191(1), 15-29, 1998.

[13] S.N.M. Ruijsenaars and H. Schneider, A New Class of Integrable Systems and Its Relation to Solitons, Annals Phys. 170, 370-405, 1986.

[14] G. Arutyunov and E. Olivucci, Hyperbolic spin Ruijsenaars-Schneider model from Poisson reduction, Proc. Steklov Inst. Math. 309 (2020), 31, [hep-th/1906.02619].

[15] G. Arutyunov, R. Klabbers and E. Olivucci, Quantum Trace Formulae for the Integrals of the Hyperbolic Ruijsenaars-Schneider model, JHEP 05 (2019), 069, [hep-th/1902.06755].

[16] H. Ochiai, T. Oshima and H. Sekiguchi, Commuting families of symmetric differential operators, Proc. Japan Acad. Ser. A Math. Sci., vol. 2, 1994, 62-66.

[17] R. P. Stanley, Some combinatorial properties of Jack symmetric functions, Adv. in Math. 77, 76-115, 1989. 
[18] J. Sekiguchi, Zonal spherical functions on some symmetric spaces, Publ. Res. Inst. Math. Sci. 12, 455-459, 1977.

[19] P.P. Kulish, Factorization of the Classical and Quantum s Matrix and Conservation Laws, Theor. Math. Phys. 26, 132, 1976, [Teor. Mat. Fiz.26,198 (1976)].

[20] C.N. Yang, Some exact results for the many body problems in one dimension with repulsive delta function interaction, Phys. Rev. Lett. 19, 1312-1314, 1967.

[21] A.B. Zamolodchikov and Al.B. Zamolodchikov, Factorized S Matrices in Two-Dimensions as the Exact Solutions of Certain Relativistic Quantum Field Models, Annals Phys. 120, 253-291, 1979.

[22] V.E. Korepin, N.M. Bogoliubov, and A.G. Izergin, Quantum Inverse Scattering Method and Correlation Functions, Cambridge Monographs on Mathematical Physics, Cambridge University Press, 1997.

[23] N.J. Zabusky, M.D. Kruskal, Interaction of "Solitons" in a Collisionless Plasma and the Recurrence of Initial States, Phys. Rev. Lett. 15, 240 (1965).

[24] L.D. Faddeev and L.A. Takhtajan, Hamiltonian Methods in the Theory of Solitons, SpringerVerlag, Berlin Heidelberg, 1987.

[25] B.A. Dubrovin, I.M. Krichever and S.P. Novikov, Integrable systems I, In: Arnold, V.I. and Novikov, S.P. (eds.) Dynamical Systems IV: Symplectic geometry and its applications, 1993. 